\title{
Market Structure and Equilibrium in a Hydro Dominated Electricity Market
}

\author{
By \\ Andrea. Y. Lu
}

\begin{abstract}
A thesis
submitted to the Victoria University of Wellington in fulfillment of the requirements for the degree of Master of Arts in Economics
\end{abstract}

Victoria University of Wellington 


\section{Acknowledgement}

I am greatly indebted to my supervisors Prof Graeme Guthrie and Prof Lewis Evans for their consistent guidance and encouragement throughout the process.

I wish to thank Brian Bull from the New Zealand Electricity Commission for providing the data on the New Zealand electricity price and water inflow, and Neil Walbran from Neil Walbran Consulting for his help in finding the data.

I would also like to sincerely thank the examiners for their invaluable comments and suggestions.

The paper has benefitted from comments of participants in seminars in the School of Economics and Finance, Victoria University of Wellington and the Western Economics Association 95 $5^{\text {th }}$ Annual Meeting.

Last but not least I would like to thank my parents for their support and James Key for his invaluable help in proof reading this thesis. 


\section{Table of Contents}

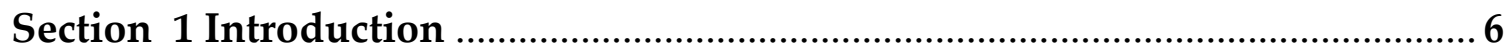

\section{Essay 2: An electricity market model}

Section 2 Model set-up ............................................................................. 9

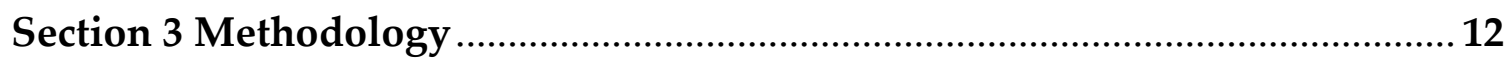

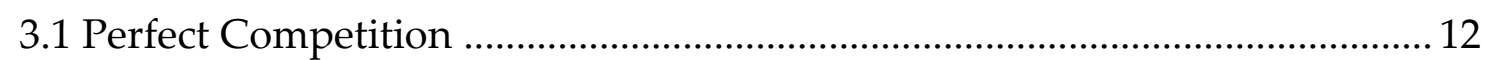

3.1.1 Social planner's objective function ........................................................... 12

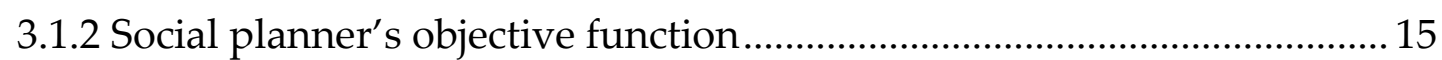

3.1.3 Numerical solution method for solving the optimal generation policy

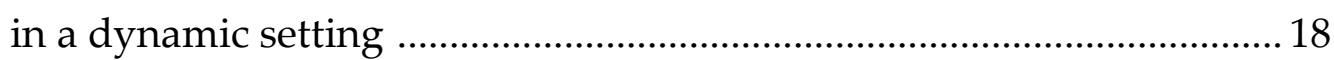

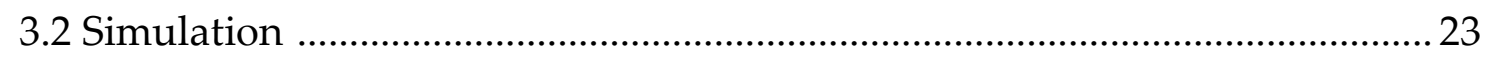

3.3 Monopolistic market ............................................................................. 24

Section 4 Model calibration for the New Zealand electricity market ............. 26

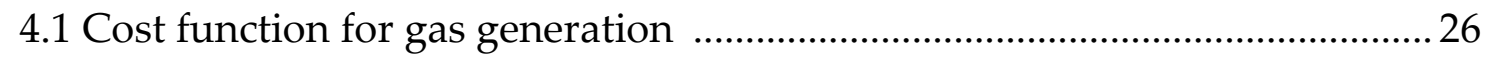

4.2 Maximum generating capacity for hydro generation and non-hydro

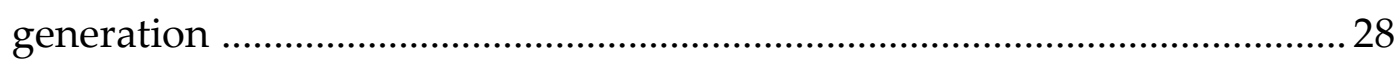

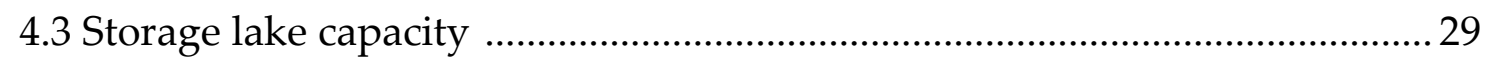

4.4 Transmission cost of hydro generated electricity and of non-hydro

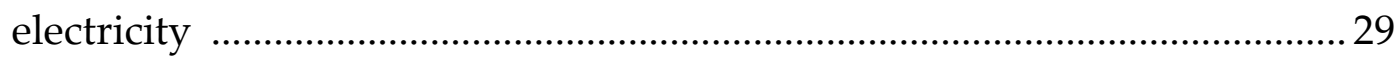

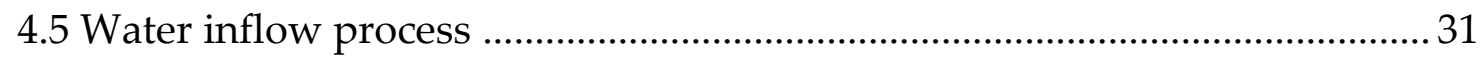

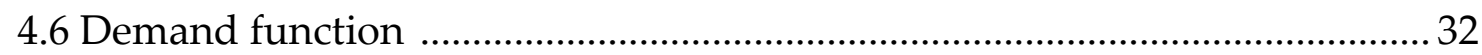

4.7 Summary of the input parameters in the New Zealand calibration ............. 33

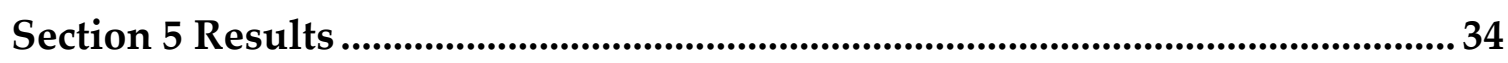

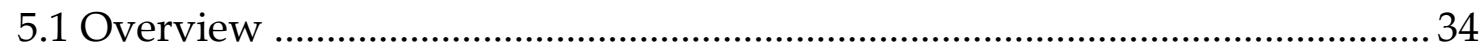

5.2 Results of the standard competitive model ………………………………...... 35

5.2.1 Static optimization results .............................................................................. 35

5.2.2 Determining the shadow price of water ....................................................... 38

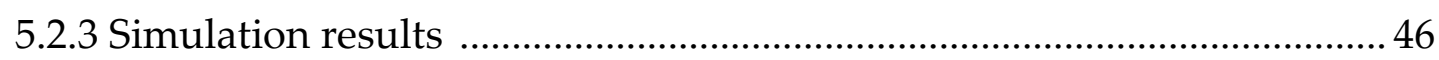

5.3 Results of the standard monopoly model ........................................................5 57 


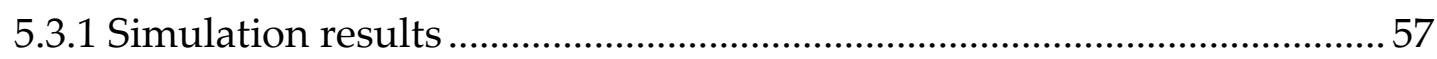

5.4 Comparison between the competitive model and the monopoly model .... 66

5.4.1 Market outcomes 66

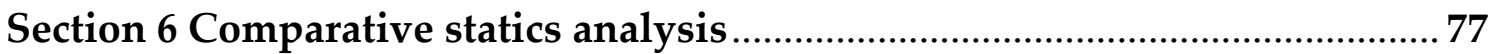

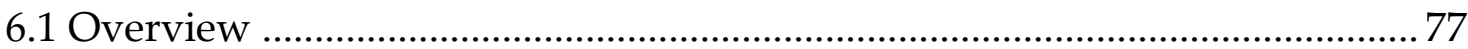

6.2 Comparative statics of the competitive model .......................................... 77

6.2.1 Capacity of the gas generation plant .................................................... 77

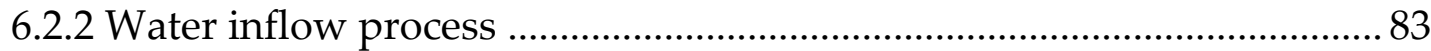

6.2.2-1 Mean of water inflow process ……................................................ 84

6.2.2-2 Mean reversion rate of water inflow process ................................ 88

\section{Essay 2: Application in Evaluating Electricity Market Power Assessment}

Section 7 Methods for assessing the exercise of market power in electricity markets

7.1 Literature review on the methods for assessing the exercise of market power in electricity markets ...................................................................... 91

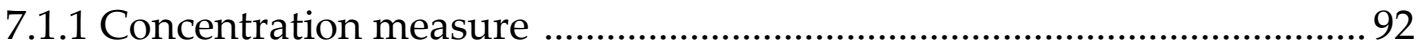

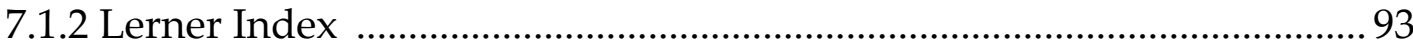

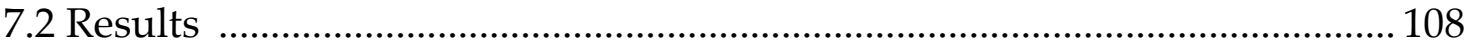

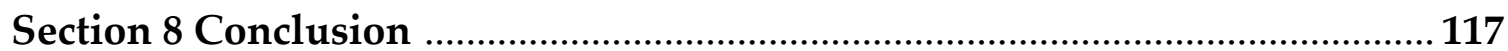

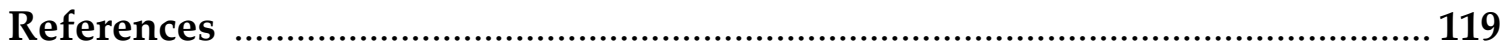

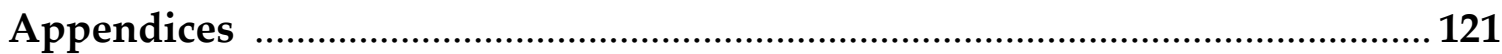

Appendix 1: Derivation of Hamilton-Jacobi-Bellman equation .............................. 121

Appendix 2: Initial guess for the generation policy …..................................... 123

Appendix 3: Evaluating Shadow prices of water Ws for points on the Right and Left boundaries 129 
Appendix 4: Estimating the parameters for water inflow that follows squareroot process 132

Appendix 5: Static optimization results for the competition model 136

Appendix 6: Static optimization result for the monopoly model 141 


\section{Section 1: Introduction}

Hydro generation plays an important role in electricity generation, especially in countries like New Zealand where 60 to 65 percent of electricity is generated in the hydro sector. In contrast to other types of electricity generation, for example gas generation, hydro generation has two unique properties: uncertainty regarding future resource availability and the ability to store the nature resource. Although hydro resource is often considered to be 'free', the ability to store creates an endogenous hidden marginal cost of water: usage today entails the loss of the ability to be used in future periods. Therefore pricing in a hydro dominated electricity market should be different from the approaches applied in markets that consist of generation methods that use only non-storable resources. This paper introduces a tractable approach to model a hydro dominated electricity market that incorporates inter-temporal decision making. It enables us to compute the equilibrium outcomes and the endogenous hidden marginal cost of water under different market structures.

There are many other works that focus on modelling the electricity market. A popular approach is to compare the actual offers of individual generators with estimates of the operational marginal cost of turning fuel into electricity. Works along this line include Boreinstein, Bushnell and Wolak(2002), Wolak (2002), Wolfram(1998), Joskow and Kahn(2002). Boreinstein, Bushnell and Wolak (1998), Wolak(2002), and Joskow and Kahn (2002) study the California wholesale electricity market; whereas Wolfram (1998) investigates in British electricity spot market. A problem with this approach is that the estimates of the operational marginal cost are computed in a static setting assuming full information. This approach does not work in hydro dominated electricity markets like the New Zealand Electricity Market where there is uncertainty regarding fuel/water availability and ability to store fuel/water over time. Many real option related works have shown that the ability to a store nature resource creates a hidden 
option value. Evans and Guthrie (2009) show that uncertainty about resource factors may materially affect generator behaviours. However, many regulators still apply the operational marginal cost approach to detect the existence of market power in electricity markets, including the ones which are hydrodominated. An observation of price exceeding the operational marginal cost (estimated in a static setting), without taken into account the hidden option value, is regarded as evidence that the generators are abusing their market power. Our paper also shows that under certain settings, the presence of real options embedded in the stored fuel make it difficult to detect abuses of market power in practice.

There are some operations research works that use dynamic decision making approach to model electricity market. The most relevant works include Scott and Read (1996) and Hogan, Read and Ring (1996). These works simulate an electricity market and model electricity generators' behaviour under a dynamic setting. However, most of these works are considerably complex and difficult to calibrate and implement. Additionally, their discrete-time model in contrast to our period-by-period optimization approach does not incorporate the real dynamic of the uncertainty in the natural resource.

Another class of literature uses Bayesian Nash Equilibrium to model electricity prices. These models build on an equilibrium framework to explain electricity spot prices in terms of observable state variables and then using non-arbitrage condition to value derivatives. Hortacsu and Puller (2005) and Hortacsu and Puller (2008) are examples of this line of research. They develop a single-period equilibrium model to model electricity spot prices under a oligopoly market structure. While these approaches are forward looking, they are difficult to calibrate. Unlike our model, they do not permit study of markets where generators use different fuels. 
Our model is calibrated to the New Zealand electricity market using publicly available data to take into account the property of hydro dominance, volatile inflow and low capacity of lake storage. It is tractable enough such that it can be easily modified to allow calibration. In this thesis we consider two polar cases of market structure - a competitive market and monopoly. The comparison between the two allows us to assess whether market structure is important for the effect of changes in properties of the nature resource.

The thesis is organized as follows. In section 2 we set up the model. In section 3 we present the methodology for implementing the model in a competitive market setting and in a monopoly market setting. In section 4 we calibrate the model to the New Zealand Electricity Market. Section 5 presents and discusses the results. In section 6 we change a few nature related inputs of the model and assess how these affect market performance. We will investigate the performance of the commonly used market power tests in section 7. Conclusions will be drawn in section 8 . 


\section{Section 2: Model set-up}

There are two types of agents in our model. Producers who generate electricity then sell it in a spot market; and consumers who purchase electricity at the market determined price.

The producer owns two types of electricity generation plants: a hydro plant and a gas plant. One firm owning multiple types of generation plants is a common scenario in New Zealand. The qualities of electricity produced by both plants are identical, however the costs associated with the different plants are not the same.

There is a limit on the amount of hydro resource that is available at each point in time, given by current inflows plus stored inflows. The hydro generation plant has the ability to store its input resources (i.e. water) over time in the storage lake. This allows the producer to allocate hydro energy across time.

Nature generates water inflow to the storage lake $y_{t}$ according to an Ito process. It is constructed from a drift function $v\left(y_{t}\right)$, a volatility function $\phi\left(y_{t}\right)$, and a Wiener Process $\zeta$. It is

$$
d y_{t}=v\left(y_{t}\right) d t+\phi\left(y_{t}\right) d \zeta_{t}
$$

Given the amount of water inflow, the producer decides how much of it to use to generate electricity immediately and how much to store in the lake to save it for future generation. Let $s_{t}$ denote the amount of water stored in the lake at time t. The lake level $s_{t}$ must satisfy the condition that $0 \leq s_{t} \leq \bar{s}$, where $\bar{s}$ is the maximum storage capacity. In other words, the amount of water in the lake has to be non-negative and cannot exceed the maximum capacity. 
In each period, the producer will choose the amount of electricity generated by each plant. Assuming that each unit of water/gas generates exactly one unit of electricity (for gas generation, this is equivalent to having a heat rate equal to one), we denote the amount of water used in generating electricity (which is equivalent to the number of units of electricity being generated using hydro energy) by $z_{t}$, and denote the amount of gas used in generation at time $t$ by $m_{t}$. Both hydro generation and the gas generation have a maximum capacity constraint on the amount of generation that can be generated in each period, denoted by $\bar{z}$ and $\bar{m}$. At any given period $t$, the actual generation must be less or equal to the capacity constraint, that is,

$$
\begin{aligned}
& z_{t} \leq \bar{z} \\
& m_{t} \leq \bar{m}
\end{aligned}
$$

It is assumed that the lake level will not be changed by any external factors apart from natural inflow and electricity generation related withdrawal. Specifically, no water is lost through evaporation and infiltration, although it may be spilled.

At each point in time, given the water inflow $y_{t}$ and the amount of water used to generate electricity $z_{t}$, the change in lake level equals the net difference between inflow and outflow. That is, the lake level $s_{t}$ evolves according to the following process

$$
d s_{t}=\left(y_{t}-z_{t}\right) d t
$$

We make the following assumptions about generation costs:

- The cost of converting each unit of hydro energy into electricity is zero.

- The total cost of converting $m_{t}$ units of gas into electricity is $\left(m_{t}\right)=b m_{t}^{2}$. 
Once the electricity is generated, it can be transmitted to consumers at given transmission costs where:

- The transmission cost for hydro generated electricity is $k_{1}$ per unit.

- The transmission cost for gas generated electricity is $k_{2}$ per unit.

In our model we set the transmission cost for hydro generated electricity to be higher than the transmission cost for gas generated electricity (that is, $k_{1}>k_{2}$ ). This is designed to reflect the fact that in general hydro generation plants are situated further away from the high demand location than are the gas generation plants, due to the geographical location of fuel and cost of fuel transmission.

Given the total electricity generation $\left(z_{t}+m_{t}\right)$ supplied by the producer, the market will determine the market clearing price for each unit of electricity according to a linear inverse demand function,

$$
\varphi\left(m_{t}+z_{t}\right)=c+d \cdot\left(m_{t}+z_{t}\right)
$$

This inverse demand function is assumed to be downward sloping with $\varphi^{\prime}<0$. 


\section{Section 3: Methodology}

\subsection{Perfect Competition}

In the first section, we analyze the agents' behaviour as if they are under perfect competition. In a competitive market, there is an infinite number of identical producers, each owning a hydro plant and a gas plant. Each producer chooses their generation policy to maximize their own profit. Since they all face the same profit and cost functions and expectations of the future, the decision made by one producer would be identical to decisions made by the others. Their lack of market power means that they all behave as price-takers. In equilibrium, in a perfectly competitive market, the market-clearing price that maximizes each producer's profit equals the marginal cost of producing the last unit of electricity. Thus, the market-wide aggregate generation policy (sum of each individual producer's policy) will be equivalent to the choices that would be made by a social planner whose intent is to maximize the total net social surplus.

\subsubsection{Social Planner's Objective Function}

For a particular level of hydro generation $\left(z_{t}\right)$ and gas generation $\left(m_{t}\right)$, the flow of total surplus to society equals the total benefit net of generation cost and transmission cost, that is:

$$
T S\left(z_{t}, m_{t} ; y\right)=\int_{0}^{m_{t}+z_{t}} \varphi(q) d q-C\left(m_{t}\right)-k_{1} z_{t}-k_{2} m_{t}
$$

What the social planner really cares about at time $t^{\prime}$ is the expected present value of life time total surplus starting from time $\mathrm{t}$ which we denote by $\mathrm{W}$ :

$$
W(s, y)=E_{0}\left[\int_{0}^{\infty} e^{-r t} T S\left(z\left(s_{t}, y_{t}\right), m\left(s_{t}, y_{t}\right) ; y_{t}\right) d t \mid\left(s_{0}, y_{0}\right)=(s, y)\right]
$$

We presume that the social planner owns both the hydro generation plant and the gas generation plant. At a particular point in time say at time zero, taking the 
lake level $s_{0}$ and the water inflow $y_{0}$ as given, the social planner will choose the amount of hydro energy $\left(z_{t}\right)$ and the amount of gas $\left(m_{t}\right)$ to be used in electricity generation at time $t$ such that they maximize the expected present value of life time total surplus W subject to their generation capacity constraints.

The stochastic characteristics of inflow $y_{t}$ and storage level $s_{t}$ determine the dynamic properties of this optimization problem. While the inflow evolves according to the Ito process over time, the storage level in the next period is strongly dependent on the inflow and the choice of the amount of hydro generation in this period.

We rewrite the value function $\mathrm{W}$ in terms of its corresponding Hamilton-JacobiBellman equation form ${ }^{1}$. This specifies the evolution of $W$ and enables it to be calculated.

The Hamilton-Jacobi-Bellman equation (HJB) for this maximization problem is the following:

$$
0=\max _{z, m}(y-z) \frac{\partial W}{\partial s}+v \frac{\partial W}{\partial y}+\frac{1}{2} \phi^{2} \frac{\partial^{2} W}{\partial y^{2}}-r W+T S(m, z ; y)
$$

The details as to how we obtain the Hamilton-Jacobi-Bellman equation form are presented in Appendix 1.

Take away the parts in the HJB that are independent of the choice of $\mathrm{z}$ or $\mathrm{m}$, and we are left with social planner's short-term objective function,

$$
\begin{aligned}
\max _{\mathrm{m}, \mathrm{z}} f & =\operatorname{TS}(m, z ; y)-z \frac{\partial W}{\partial s} \\
& =\int_{0}^{m+z} \varphi(q) d q-z \frac{\partial W}{\partial s}-C(m)-k_{1} z-k_{2} m
\end{aligned}
$$

\footnotetext{
${ }^{1}$ R. E. Bellman. Dynamic Programming. Princeton, NJ, 1957.
} 
In this expression, the term $\int_{0}^{m+z} \varphi(q) d q$ represents the total benefit to society which is the area underneath the demand curve for a given quantity of output $q=m+z$. The term $\frac{\partial W}{\partial s}$ is the marginal gain in the expected present value of total surplus $(W)$ given a one unit increase in storage level (s); this is what we call the "Shadow Price" of water. This shadow price represents the opportunity cost of using one more unit of water in generation as alternatively that unit of water could have been stored, which would cause the expected present value of total surplus $W$ to increase by $\frac{\partial W}{\partial s}$. Therefore $z \frac{\partial W}{\partial s}$ measures the cost of producing $z$ units of hydro-electricity, whereas $C(m)$ gives the generation cost for producing $\mathrm{m}$ units of gas-generated electricity. The terms $k_{1} z$ and $k_{2} m$ are the transmission costs for given levels of generation $\mathrm{z}$ and $\mathrm{m}$.

Hence, at time $t$, the social planner chooses the level of generation $\left(m_{t}\right.$ and $\left.z_{t}\right)$ to maximize the objective function $f$ which represents the value of total benefit to the society net of all the generation costs and transmission costs subject to generation capacity constraints.

The optimization would be straightforward if we could take the shadow price of water $\frac{\partial W}{\partial s}=W_{s}$ as given. However, the optimization problem is not as straightforward as a simple maximization problem. This is because in the dynamic setting when the inflow (y) and storage level (s) change over time, the shadow price of water is no longer exogenous. At time $t$, with water inflow $y_{t}$ and storage level $s_{t}$, the shadow price of water $\left(W_{s_{t}}\right)$ is determined by the producer's optimal generation policy $\left(z_{t}\right.$ and $\left.m_{t}\right)$ in that period. Given the amount of inflow $y_{t}$ and the optimal level of hydro generation $z_{t}$, the storage level changes by $d s_{t}$ where $d s_{t}=\left(y_{t}-z_{t}\right) d t$. As a result, storage changes to a new level $s^{\prime}$. This change causes the shadow price of water to change from $W_{s}$ to 
$W_{s}{ }^{\prime}$. Accordingly the optimal generation policy and storage policy have to be readjusted to be consistent or optimal with respect to the new shadow price. This process will iterate until $W_{s}, z$ and $m$ are consistent with the condition and there is no change.

To solve for the equilibrium generation policy and storage policy we take two steps. Firstly, we solve for the optimal generation policy in a static setting for a fixed point in time, while taking the inflow $(y)$ and the shadow price of water $\left(W_{s}\right)$ as given. Second, we find an internally consistent optimal generation policy by iterating over time to the point where the generation decisions are optimal and consistent with the observed shadow price of water.

\subsubsection{Optimal generation policy in a static setting}

As we saw in section 3.1.1, in a static setting, the optimization problem faced by the social planner is that given the exogenous inflow from the river and a lake to store they need to find the generation policy $\left(z^{*}, m^{*}\right)$ that maximizes total social welfare

$$
\max _{m_{t}, z_{t}} f=\int_{0}^{m_{t}+z_{t}} \varphi(q) d q-z_{t} \frac{\partial W}{\partial s}-C\left(m_{t}\right)-k_{1} z_{t}-k_{2} m_{t}
$$

subject to generation capacity constraints,

$$
\begin{gathered}
z_{t} \leq \bar{z} \\
m_{t} \leq \bar{m} \\
z_{t} \geq 0 \\
m_{t} \geq 0
\end{gathered}
$$

and some other additional constraints which depend on the inflow and the lake level. 
- When the lake is neither empty nor full $\left(0<S_{t}<\bar{S}\right)$, the social planner will choose the optimal levels of hydro and gas generation that maximize total social welfare subject to the constraints that the value for gas generation and for hydro generation are both non-negative and do not exceed the generation capacities $\left(0 \leq z_{t} \leq \bar{z}\right.$ and $\left.0 \leq m_{t} \leq \bar{m}\right)$. Since there is water in storage and inflow from the river, at a particular point in time there is no upper bound constraint on the availability of the water.

- When the lake is empty $\left(S_{t}=0\right)$, producer is unable to use more water to generate electricity than the amount of inflow for that particular period. This puts an upper bound on hydro generation. Therefore beside the normal non-negativity and generation capacity constraints $\left(0 \leq z_{t} \leq \bar{z}\right.$ and $\left.0 \leq m_{t} \leq \bar{m}\right)$ an additional constraint has to be imposed. That is the level of hydro generation has to be lower than the inflow from the river $\left(z_{t} \leq y_{t}\right)$ when the lake is empty.

- When the lake is full $\left(S_{t}=\bar{S}\right)$, the producer loses the ability to store water. For the amount of inflow from the river, the producer can choose either to use it in generation or to spill it. The shadow price for water equals zero when spilling takes place as if it is positive then it will be optimal to use this water in generation rather than spilling it. Therefore the social planner's objective function depends on the inflow in that period:

When there is spilling $\left(z<y_{t}\right)$

$$
f=\int_{0}^{m+z} \varphi(q) d q-C(m)-k_{1} z-k_{2} m
$$

When there is no spilling $\left(z \geq y_{t}\right)$ 


$$
f=\int_{0}^{m+z} \varphi(q) d q-C(m)-\left(z-y_{t}\right) \frac{\partial W}{\partial s}-k_{1} z-k_{2} m
$$

The social planner will choose a pair of $z$ and $m$ that maximizes the objective function subject to the non-negativity constraints, capacity constraints and the extra constraint imposed on $z_{t}$ (i.e. spilling and no spilling). The decision of whether to spill or not will depend on which one will provide a higher total social welfare.

As a result, the optimization problem faced by the social planner depends on the amount of water in the storage lake and we optimize over a set of values of $W_{S}$ and $y$. It is useful to set up a grid that contains different combinations of $W_{s}$ and $y$. For each pair of $W_{s}$ and $y$, the social planner will be able to calculate the optimal generation policies $\left(z^{*}\right.$ and $\left.m^{*}\right)$ that maximize the corresponding objective function. To reduce the calculation time, linear interpolation is employed for evaluating the optimal generation policy at combination of $W_{s}$ and $y$ that is between the points which have been calculated using the constrained optimization.

The optimal static generation policy can therefore be summarized in 6 tables

(1) Optimal hydro generation when lake is empty $\left(z^{*}\right.$ given $\left.s_{t}=0\right)$

(2) Optimal hydro generation when lake has spare capacity $\left(z^{*}\right.$ given $\left.0 \leq s_{t} \leq \bar{s}\right)$

(3) Optimal hydro generation when lake is full $\left(z^{*}\right.$ given $\left.s_{t}=\bar{s}\right)$

(4) Optimal gas generation when lake is empty $\left(m^{*}\right.$ given $\left.s_{t}=0\right)$

(5) Optimal gas generation when lake has spare capacity $\left(m^{*}\right.$ given $\left.0 \leq s_{t} \leq \bar{s}\right)$

(6) Optimal gas generation when lake is full $\left(m^{*}\right.$ given $\left.s_{t}=\bar{s}\right)$ 
These 6 tables give the set of optimal generation policies in a static setting when the shadow price of water and the inflow are taken as given. The set of tables for the competitive market setting can be found in Section 5 .

\subsubsection{Numerical solution method for solving the optimal generation policy in a dynamic setting}

We use policy iteration to solve for the social planner's optimal generation policy in the dynamic setting on a discrete grid in $(s, y)$ space.

To set up the grid, let $\mathrm{s}$ go from $s_{\min }$ to $s_{\max }\left(s_{1}<s_{2}<\cdots<s_{n+1}\right)$ with incremental size of $d s$ and let y go from $y_{\min }$ to $y_{\max }\left(y_{1}<y_{2}<\cdots<y_{m+1}\right)$ with increment size of $d y$ - we will have a discrete grid with contains $(n+1)(m+1)$ pairs of different combinations of storage levels and river inflow $y$.

The starting point is an initial guess for the social planner's generation policy as a function of storage level and river inflow, denoted as $z^{(0)}(s, y)$ and $m^{(0)}(s, y)$

$$
\begin{aligned}
& z^{(0)}(s, y)=a+b s+c y \\
& m^{(0)}(s, y)=0.2
\end{aligned}
$$

The detailed methodology on the choice of this particular initial generation policy is described in Appendix 2.

The equilibrium level of hydro generation, gas generation and storage satisfy the following Hamilton-Jacobi-Bellman (HJB) equation:

$$
0=\left(y-z^{*}\right) \frac{\partial W}{\partial S}+v \frac{\partial W}{\partial y}+\frac{1}{2} \phi^{2} \frac{\partial^{2} W}{\partial y^{2}}-r W+\int_{0}^{m^{*}+z^{*}} \varphi(q) d q-C\left(m^{*}\right)-k_{1} z^{*}-k_{2} m^{*}
$$

The finite difference method is used for approximating the first and second order differentials in the HJB equation.

We can use central difference to approximate $\frac{\partial W}{\partial y}$ and $\frac{\partial^{2} W}{\partial y^{2}}$ as in: 


$$
\begin{aligned}
& \frac{\partial W}{\partial y}\left(s_{i}, y_{j}\right)=\frac{W\left(s_{i}, y_{j+1}\right)-W\left(s_{i}, y_{j-1}\right)}{2 d y} \\
& \frac{\partial^{2} W}{\partial y^{2}}\left(s_{i}, y_{j}\right)=\frac{W\left(s_{i}, y_{j+1}\right)-2 W\left(s_{i}, y_{j}\right)+W\left(s_{i}, y_{j-1}\right)}{d y^{2}}
\end{aligned}
$$

However, the finite difference approximation of $\frac{\partial W}{\partial s}$ depends on the sign of $\left(y-z^{*}\right)$. Given the storage level s and river inflow $y$, when the optimal level of hydro generation is less than the amount of inflow from the lake it is optimal for the social planner to increase the storage level, so we use the forward difference to approximate the shadow price of water $W_{s}$. However, if the optimal level of hydro generation is higher than the inflow $\mathrm{y}$, then the backward difference will be used.

That is:

When $z^{*}<y$, the HJB equation is approximated by

$$
\begin{aligned}
0=\left(y-z^{*}\right) & \frac{W\left(s_{i+1}, y_{j}\right)-W\left(s_{i}, y_{j}\right)}{d s}+v \frac{W\left(s_{i}, y_{j+1}\right)-W\left(s_{i}, y_{j-1}\right)}{2 d y} \\
& +\frac{1}{2} \phi^{2} \frac{W\left(s_{i}, y_{j+1}\right)-2 W\left(s_{i}, y_{j}\right)+W\left(s_{i}, y_{j-1}\right)}{d y^{2}}-r W\left(s_{i}, y_{j}\right) \\
& +\int_{0}^{m^{*}+z^{*}} \varphi(q) d q-C\left(m^{*}\right)-k_{1} z^{*}-k^{2} m^{*}
\end{aligned}
$$

at all interior nodes $0 \leq i \leq n, 0 \leq j \leq m$. 
When $z^{*} \geq y$, it is approximated by

$$
\begin{aligned}
0=\left(y-z^{*}\right) & \frac{W\left(s_{i}, y_{j}\right)-W\left(s_{i-1}, y_{j}\right)}{d s}+v \frac{W\left(s_{i}, y_{j+1}\right)-W\left(s_{i}, y_{j-1}\right)}{2 d y} \\
& +\frac{1}{2} \phi^{2} \frac{W\left(s_{i}, y_{j+1}\right)-2 W\left(s_{i}, y_{j}\right)+W\left(s_{i}, y_{j-1}\right)}{d y^{2}}-r W\left(s_{i}, y_{j}\right) \\
& +\int_{0}^{m^{*}+z^{*}} \varphi(q) d q-C\left(m^{*}\right)-k_{1} z^{*}-k^{2} m^{*}
\end{aligned}
$$

at all interior nodes $0 \leq i \leq n, 0 \leq j \leq m$.

Given the initial guess for the generation policy, we can set up the interior equations after making the substitution $z^{*}=\mathrm{z}^{(0)}$ and $m^{*}=m^{(0)}$. There is one equation for each pair of $s$ and $y$, for values of $s_{i}$ goes from $s_{2}$ to $s_{n}$ and for $y_{j}$ goes from $y_{2}$ to $y_{m}$. Thus there will be in total $\mathrm{nm}$ values.

When the storage lake is full $\left(s_{i}=s_{n+1}\right)$, it is impossible for the generators to store any more water in the lake. In such case, if the inflow is higher than the optimal level of hydro generation, the excess inflow has not value as it cannot be stored for future use. $\frac{\partial W}{\partial s}$ has a positive value only when the optimal hydro generation is greater or equal to the inflow in that period. Therefore, we impose a numerical boundary condition along the right boundary of the grid as follows:

when $s_{i}=s_{n+1}$, then

when $\mathrm{z}^{*}<y_{j}$

$$
\begin{gathered}
0=v \frac{W\left(s_{i}, y_{j+1}\right)-W\left(s_{i}, y_{j-1}\right)}{2 d y}+\frac{1}{2} \phi^{2} \frac{W\left(s_{i}, y_{j+1}\right)-2 W\left(s_{i}, y_{j}\right)+W\left(s_{i}, y_{j-1}\right)}{d y^{2}} \\
-r W\left(s_{i}, y_{j}\right)+\int_{0}^{m^{*}+z^{*}} \varphi(q) d q-C\left(m^{*}\right)-k_{1} z^{*}-k^{2} m^{*}
\end{gathered}
$$


and when $z^{*} \geq y_{j}$

$$
\begin{aligned}
0=\left(y_{j}-z^{*}\right) & \frac{W\left(s_{i}, y_{j}\right)-W\left(s_{i-1}, y_{j}\right)}{d s}+v \frac{W\left(s_{i}, y_{j+1}\right)-W\left(s_{i}, y_{j-1}\right)}{2 d y} \\
& +\frac{1}{2} \phi^{2} \frac{W\left(s_{i}, y_{j+1}\right)-2 W\left(s_{i}, y_{j}\right)+W\left(s_{i}, y_{j-1}\right)}{d y^{2}}-r W\left(s_{i}, y_{j}\right) \\
& +\int_{0}^{m^{*}+z^{*}} \varphi(q) d q-C\left(m^{*}\right)-k_{1} z^{*}-k^{2} m^{*}
\end{aligned}
$$

for $y_{j}$ from $y_{2}$ to $y_{m}$.

Additionally, we impose numerical boundary conditions along the top and bottom boundaries of the grid (for $y$ ):

For all values of storage level $\left(s_{i}\right)$,

$$
\begin{aligned}
& W\left(s_{i}, y_{m-2}\right)-2 W\left(s_{i}, y_{m-1}\right)+W\left(s_{i}, y_{m}\right)=0 \\
& W\left(s_{i}, y_{1}\right)-2 W\left(s_{i}, y_{2}\right)+W\left(s_{i}, y_{3}\right)=0 .
\end{aligned}
$$

The finite difference equations together with the numerical boundary conditions (see Appendix 3) enable us to solve for the finite difference approximation to the social planner's objective function for each pair of $s$ and $y$ in the grid, which is denoted by $W^{(n)}(s, y)$.

The shadow price of water $W_{s}$ can be evaluated by using the finite difference approximation at each point $(s, y)$ in the grid. Evaluating the shadow price for points inside the grid that are not on the left or right boundary is quite straight forward. The standard central difference approximation can be used for these:

$$
W_{s}\left(s_{i}, y_{j}\right)=\frac{W\left(s_{i+1}, y_{j}\right)-W\left(s_{i-1}, y_{j}\right)}{2 d s}
$$


However, for the combination of $\mathrm{s}$ and $\mathrm{y}$ that are lying on the left and right boundaries evaluating $W_{s}$ at those points is a little bit more complicated. For the points along the left boundary, $s_{i}=s_{1}$. Since $s_{i}$ is already at the lowest value for $\mathrm{s}, s_{i-1}$ does not exist; whereas the points lying on the right hand side boundary, $s_{i}$ already represent the highest value for $\mathrm{s}$ therefore there is no value for $s_{i+1}$. As a result, different formulas have to be adopted to evaluate the shadow price $W_{s}$ when $s$ is at its lowest or highest level.

For $s_{i}=s_{\min }$

$$
W_{s}\left(s_{i}, y_{j}\right)=\frac{4 W\left(s_{i+1}, y_{j}\right)-W\left(s_{i+2}, y_{j}\right)-3 W\left(s_{i}, y_{j}\right)}{2 d s}
$$

Fors $s_{i}=s_{\max }$

$$
W_{s}\left(s_{i}, y_{j}\right)=\frac{4 W\left(s_{i}, y_{j}\right)-W\left(s_{i-1}, y_{j}\right)-3 W\left(s_{i-2}, y_{j}\right)}{2 d s}
$$

To see the derivation of these two formulas, see Appendix $3^{2}$.

After evaluating the shadow price for each point on the grid, taking the shadow price $W_{s}$ and $y$ as given we can use the optimization table we obtained in A.1 to update the optimal generation policy $z^{n+1}(s, y)$ and $m^{n+1}(s, y)$ which is further substituted into the HJB equations in order to solve for the new $W$ and to evaluate the new value for $W_{s}$. We repeat this sequence of steps until convergence occurs.

\footnotetext{
${ }^{2}$ Values of $W_{s}$ at the boundaries are calculated using the two available adjacent values to create an extrapolated value on the other side, by way of a second-order Taylor approximation.
} 


\subsection{Simulation}

Using the approach described in section 3.1, we can obtain the optimal generating policy and the optimal storage policy in a competitive market, for each combination of water inflow y and storage level s:

- Optimal Generating Policy $G C(s, y)=\left\{z^{*}, m^{*} \mid(s, y)\right\}$

This policy gives us the optimal gas and hydro generation given $(s, y)$.

- Optimal Storage Policy $S C(s, y)=\left\{d s, W_{s} \mid(s, y)\right\}$

This policy tells us about the direction and magnitude of the change in the storage lake level and the shadow price of water $W_{s}$ given $(s, y)$.

To model the price behaviour in a competitive market, we use Monte Carlo simulation to simulate a series of water inflows $y_{t}$ across time. With the given series of simulated inflows we are able to compute the optimal generating policy and optimal storage policy. Knowing the initial value of storage and how water inflows evolves over time, we can use the two policies that we obtained in section 3.1 to understand producer's optimal behaviour in a perfectly competitive market which includes the equilibrium market clearing price path, the amount of electricity generated by the gas plant and the amount generated by the hydro plant. 


\subsection{Monopoly market}

When the market has a monopoly structure there is only one producer in the market. The monopolist chooses the amount of gas generation and hydro generation to maximize his own profit. Since he is the only producer in the market, he represents the market supply and has full influence over the market price. In other words, he is a price-maker. Therefore at equilibrium, the market clearing price in a monopoly market will be the price that maximizes profit $\Pi$ to the monopolist instead of the one that maximizes total net social surplus TS, where

$$
\Pi\left(z_{t}, m_{t} ; y\right)=\varphi\left(m_{t}+z_{t}\right) \cdot\left(m_{t}+z_{t}\right)-C\left(m_{t}\right)-k_{1} z_{t}-k_{2} m_{t}
$$

In a dynamic setting, the monopolist will choose a policy that maximize the expected present value of life time profit $Z$,

$$
Z(s, y)=E_{0}\left[\int_{0}^{\infty} e^{-r t} \Pi\left(z_{t}\left(s_{t}, y_{t}\right), m_{t}\left(s_{t}, y_{t}\right) ; y_{t}\right) d t \mid\left(s_{0}, y_{0}\right)=(s, y)\right]
$$

The corresponding Hamilton-Jacobi-Bellman equation for this maximization problem is in the following form

$$
0=\max _{m, z}(y-z) \frac{\partial z}{\partial s}+v \frac{\partial z}{\partial y}+\frac{1}{2} \phi^{2} \frac{\partial^{2} z}{\partial y^{2}}-r Z+\Pi(m, z ; y)
$$

The objective function $f$ that the monopolist maximizes is in the following form,

$$
\max _{m, z} f=\varphi(m+z) \cdot(m+z)-z \frac{\partial W}{\partial s}-C(m)-k_{1} z-k_{2} m
$$

The rest of the procedures to model the optimal behaviour of the monopolist follow the same steps as the perfect competition case (described in section 3.1). 
We can obtain the optimal generating policy and the optimal storage policy in the monopolistic market, at given inflow and storage levels. Then using these two optimal policies along with simulated water inflow $y_{t}$ we can find out the market clearing price path, the amount of electricity generated by the gas plant and the amount generated by the hydro plant chosen by the monopolist in dynamic equilibrium. 


\section{Section 4: Model calibration for New Zealand Electricity Market}

This section presents the calibration we apply to the model described in section 2 to capture relevant characteristics of the New Zealand Electricity Market. The data we use to estimate the parameters in our model are mostly available from public websites except the data on water inflow which is provided by New Zealand Electricity Commission ${ }^{3}$. For simplicity in calculation, we convert all generation quantity estimates into units of "standard unit", where one standard unit of electricity energy equals to 24727 gigawatt hours (which is the long run unconditional mean of water inflow). Additionally, all dollar amounts are measured in units of a billion dollars.

\subsection{Cost function for gas generation}

It is assumed that the cost function for non-hydro generation in New Zealand takes the following form $C(m)=b m^{2}$. At each generation quantity of $m$, the marginal cost of generating this quantity equals $2 \mathrm{bm}$. Therefore in our model the non-hydro generation has an increasing marginal cost function where the term $b$ determines the rate of increase in the marginal cost of non-hydro generation.

In 2007, total electricity generated by non-hydro generation is 19091 gigawatt hours ${ }^{4}$ which is equivalent to 0.77207 standard units of generation. For simplicity, though unrealistic, we assume that the only non-hydro generation option is gas combined cycle. Data show that, the short run marginal cost of gas combined cycle is at about $\$ 56$ per megawatt hour if fuel of $\$ 7$ per GJ is used and zero carbon cost; $\$ 90$ per megawatt hour if using fuel that costs $\$ 10$ per GJ and a carbon cost of $\$ 30$ each tonne; and $\$ 119$ per megawatt hour if the expensive fuel

\footnotetext{
${ }^{3}$ Inflow data available from the centralised dataset issued by the New Zealand Electricity Commission.

${ }^{4}$ http;//www.med.govt.nz/upload/59482/00_EDF-June2008.pdf 
of $\$ 13$ per GJ is used with carbon cost of $\$ 50$ per tonne ${ }^{5}$. If the medium level of marginal cost of gas generation is taken as an estimate for the marginal cost of non-hydro generation, the short run marginal cost of generating one standard unit of generation (24727 gigawatt hours) equals 2.22543 billion dollars. Given the quantity of non-hydro generation in 2007 and the marginal cost of generation, we calculate the value for the parameter $b$ in the cost function of gas generation, to be 1.44121. And the cost function of non-hydro generation is $C(m)=$ $1.44121 \mathrm{~m}^{2}$. However, one problem is that the quantity of non-hydro generation that we use in the calculation above corresponds to the market clearing quantity of all no-hydro generation at the market clearing price of $\$ 48.5$ per megawatt hours, which is equivalent to $\$ 1.19926$ billion per standard unit. If the marginal cost of $\$ 90$ was used (equivalent to 2.22543 billion dollars per standard unit), then the marginal cost of producing the market clearing quantity of non-hydro generated electricity will be higher than its corresponding price. This would lead to a lower quantity of non-hydro generated electricity at equilibrium compared to the market observed level. This is because in the real world apart from gas generation there are many other types of non-hydro generation. Many of these other generation facilities have lower marginal cost of generation than gas plant. Using the medium level of marginal cost of gas generation to represent the marginal cost at the total quantity generated by all non-hydro generations (which includes not only quantities generated by gas plant but also quantities generated by other non-hydro plants) cause an over-estimate of the marginal cost of non-hydro generation.

Knowing that the New Zealand electricity market has an oligopoly structure, at equilibrium the amount of electricity generated using non-hydro generation is likely to be smaller than the quantity that would have been generated in a

\footnotetext{
${ }^{5}$ Short run marginal cost estimates of Taranaki Combined Cycle generation http://www.electricity commission.govt.nz/pdfs/opdev/transmis/soo/pdfssoo/2008/Final2008SOO.pdf
} 
perfectly competitive market. When the market is perfectly competitive price equals marginal cost at the market clearing quantity. Therefore at the market clearing quantity, marginal cost of producing such quantity has to be no higher than the price. Therefore, the market clearing price of electricity in 2007 (1.19926 billion dollars) can serve as an upper bound for the marginal cost of non-hydro generation at its corresponding market clearing quantity of 0.77207 standard unit. Using this information, we calculate the value for $b$ to be 0.7112 .

The corresponding cost function of non-hydro generation is,

$$
C(m)=0.7112 m^{2}
$$

In our model we assume that the non-hydro generation has the cost function in the form above. This assumption biases our estimate of marginal cost upwards, and therefore our estimates of price-cost margins downwards.

\subsection{Maximum generating capacity for hydro generation and non-hydro generation}

In December 2007, the total installed generating capacity of New Zealand (including all sources) was 9133 megawatts. Out of this, 5366 megawatts is provided by hydro generating plant and the rest of 3767 megawatts belong to the non-hydro generating plant. ${ }^{6}$

Assuming that both of the hydro generating plant and the non-hydro generating plant operate twenty-four hours a day, three hundred sixty-five days a year, the maximum quantity of electricity that can be generated from hydro each year is approximately 47006.2 gigawatt hours which is equivalent to 1.901 standard

\footnotetext{
${ }^{6}$ http://www.med.govt.nz/upload/59482/00_EDF-June2008.pdf
} 
units; and the maximum quantity of electricity that can be generated by nonhydro generating plant in a year is 32998.9 gigawatt hours, which is equivalent to 1.33454 standard units.

Therefore, each year the maximum capacity of hydro generation is 1.901 standard units and the maximum capacity of non-hydro generation is 1.33454 standard units.

\subsection{Storage lake capacity}

In 26 June 2006, the total amount of hydro energy stored in the storage lakes in New Zealand was 2399 gigawatt hours, which is about $54 \%$ of maximum capacity. ${ }^{7}$ Therefore the maximum amount of hydro energy that can be stored in the storage lake is 4442.6 gigawatt hours which is equivalent to 0.17967 standard units.

\subsection{Transmission cost of hydro generated electricity and of non-hydro electricity}

For simplicity, in the model we assumed that there is only one hydro generating plant and one non-hydro (gas) generating plant. This assumption makes it difficult for us to find real data that tell us the transmission cost for the two plants in our model, as in the real world there are multiple generating plants of each kind and each plant has its own transmission cost which depends on its own geographical location. On the other hand, the amount of data available on the transmission cost is very limited. Therefore it is challenging to find a cost value for transmitting hydro generated electricity, similarly for non-hydro generated electricity. In our calibration, we use the difference between the price

\footnotetext{
${ }^{7}$ http://www.electricityinfo.co.nz/media_releases/Hydrological\%20Summary.pdf 
of electricity in a high consumption area and the price at one chosen generation node to approximate the cost of transmitting each unit of electricity from its generating plant to the end users ${ }^{8}$.

We choose the price of electricity at the Otahuhu node as an approximation of the price of electricity at consumer demand's end. The reason for choosing Otahuhu is that the majority of total electricity demand in New Zealand is from the northern North Island area, particularly the Auckland region, and Otahuhu is the plant that is closest to the main demand area. The cost of transmitting electricity generated at Otahuhu to end users is small enough so that the price at the Otahuhu node can serve as a good estimate for the price to consumers.

The majority of the hydro resources are in the South Island. Electricity generated by the hydro plants in South Island have to be transmitted through an interisland HVDC link across Cook Strait all the way to the main demand area in North Island, which leads to a high transmission cost. To capture this feature we use the price at Benmore node to represent the price of electricity at the hydrogenerating plant before transmission.

On the other hand, unlike hydro-generating plant, gas plants have more flexibility in choosing their location as they are less dependent on geographical resources. As a result, most of the non-hydro generating plants are built in North Island to avoid the high cost of transmitting across inter-island HVDC link. Therefore we choose the price at the Haywards node as an approximation for the price of non-hydro generated electricity pre transmission.

Data show that on average the difference between the price at Otahuhu node and price at Benmore node is about $\$ 7941$ per gigawatt hour. The cost of

\footnotetext{
${ }^{8}$ The nodes whose prices are used to calibrate different aspects of the model are the standard three nodes used in the New Zealand literature as representative of the entire market.
} 
transmitting 24727 gigawatt hours of hydro generated electricity is $\$ 196,357,107$, that is the transmission cost of transmitting one standard unit of hydro generated electricity to its end users is about 0.19636 billion dollars.

On the other hand, by comparing the price at Otahuhu with the price at Haywards, we find that on average for each gigawatt hour of electricity the price at Otahuhu is about $\$ 4087$ higher than the price at Haywards. The cost of transmitting 24727 gigawatt hours of non-hydro generated electricity is $\$ 101,059,249$. In other words the transmission cost of non-hydro generated electricity is about 0.10106 billion dollars per standard unit.

\subsection{Water inflow process}

It is assumed that water inflow follows the square-root process, that is,

$$
d y_{t}=\eta\left(\mu-y_{t}\right) d t+\sigma \sqrt{y_{t}} d \zeta_{t}
$$

We estimate the parameters for the water inflow $\left(y_{t}\right)$ using the monthly water inflow data available from the centralised dataset issued by the New Zealand Electricity Commission. The original data are measured in gigawatt hours, after converting them into standard units, using the approach described in Appendix 4 , by fitting the square root model to monthly the water inflow data and we find that, for the period from July 1931 to June 2006',

$$
d y_{t}=6.9448\left(1-y_{t}\right) d t+0.9056 \sqrt{y_{t}} d \zeta_{t}
$$

This fitted process gives a mean of 1 and standard deviation of 0.243 .

\footnotetext{
${ }^{9}$ The number of observations used in the regression is 899 . The adjusted R-square of the regression is 0.982 .
} 


\subsection{Demand function}

In our model we choose to use a linear demand function taking the following form $P(q)=a-b q$, where $a$ and $b$ are both constant.

This is different from the demand function that Borenstein and Bushnell used for the California electricity wholesale market which takes the following form:

$$
P(q)=c q^{-\frac{1}{\varepsilon}}
$$

where $\mathrm{P}$ is the market price, $\mathrm{q}$ the market demand, and $\varepsilon$ the price elasticity of demand ${ }^{10}$. The reason for not using the curved demand function is that it has the same price elasticity of demand at all different prices and quantities whereas for the linear demand price elasticity increases as the quantity gets larger. This linear demand function enables us to take into account the changes in consumer's behaviour along the demand function.

To estimate the value for the parameters $a$ and $b$, we conduct the following calculations. We take the average value of electricity prices at Otahuhu, Haywards and Benmore as an estimate for the equilibrium price of electricity in New Zealand in the year of 2007 , which gives $\$ 48.5$ per megawatt hour. That is, the price of each standard unit of electricity is about $\$ 1.19926$ billion. In the same year, 42374 gigawatt hours of electricity were generated in New Zealand, which is equivalent to 1.7136733 standard units. We set the price elasticity of demand at the market clearing price and quantity, equal to 0.4, which is the value Boreistein and Bushnell used in their 1998 paper $^{11}$. Using this information above we calibrate the parameters in the demand function. Therefore the demand function for the New Zealand electricity market is,

$$
P(q)=4.19741-1.74955 q ;
$$

\footnotetext{
${ }^{10}$ All of the price elasticity of demand we used in this thesis are measured in its absolute value.

11 Boreistein and Bushnell (1998).
} 
4.7 Summary of the input parameters in the New Zealand calibration

\begin{tabular}{|c|c|}
\hline Cost function of gas generation & $C(m)=0.7112 m^{2}$ \\
\hline Hydro generation capacity & 1.901 standard units \\
\hline Non-hydro generation capacity & 1.33454 standard unit \\
\hline Storage lake capacity & 0.17967 standard units \\
\hline $\begin{array}{l}\text { Transmission cost of hydro } \\
\text { generated electricity ( k1) }\end{array}$ & 0.19636 billion dollars per standard unit \\
\hline $\begin{array}{l}\text { Transmission cost of non-hydro } \\
\text { generated electricity ( k2) }\end{array}$ & 0.10106 billion dollars per standard unit \\
\hline Water inflow process & $d y_{t}=6.9448\left(1-y_{t}\right) d t+0.9056 \sqrt{y_{t}} d \zeta_{t}$ \\
\hline $\begin{array}{l}\text { Demand function } \\
\text { (when } \epsilon=0.4 \text { ) }\end{array}$ & $P(q)=4.19741-1.74955 q$ \\
\hline
\end{tabular}




\section{Section 5 Results}

\subsection{Overview}

In the previous section, we calibrated the model presented in Section 2 to capture some key characteristics of the New Zealand Electricity Market. The market outcomes given a certain market structure (perfect competition or monopoly) can be found by solving the calibrated model using the methodology described in Section 3. This section presents the results.

Section 5.2 of this section presents the results from the standard competitive model. This section is divided into three parts. Section 5.2.1 presents the optimization results in a static setting. These results describe the optimal generation policies, given levels of inflow and the shadow price of water. Section 5.2.2 presents the results of rendering the shadow price of water endogenous in a dynamic setting. It describes the optimal storage policy, generation policy and price behaviour at each given level of inflow and storage. Section 5.2.3 presents the outcomes of a competitive market given a simulated series of 30 years of daily water inflow data.

Section 5.3 presents the results from the standard monopoly model. These results are presented in the same way as those of the competitive model. Section 5.3.1 presents the optimization results in a static setting, and the dynamic optimization results are presented in section 5.3.2. Section 5.3.3 gives the results from the simulation.

Finally in section 5.4, we will compare the results from the standard competitive model and from the standard monopoly model to see the difference between their generation policies, storage policies, price behaviours and other results. 


\subsection{Results of the standard competitive model}

\subsubsection{Static optimization results}

As mentioned in section 3.1.2, in a static setting - the social planner chooses the amount of hydro and gas generation to maximize its objective function while the water inflow and the shadow price of water are taken as given. The objective function for the social planner is,

$$
\max _{m, z} f=\int_{0}^{m+z} \varphi(q) d q-C(m)-\left(z-y_{t}\right) \frac{\partial W}{\partial s}-k_{1} z-k_{2} m
$$

The results of this approach are in accord with those of a (perfectly) competitive market where generators have no ability to affect prices. The social planner's optimal or competitive markets' equilibrium generation policies in this static setting can be summarized by the following six diagrams, which cover the optimal generation policy for both hydro and gas generation while the lake is empty, or full, or intermediate between these boundaries.

(1) Optimal hydro generation when the lake is empty $\left(z * \mid s_{t}=0\right)$;

(2) Optimal hydro generation when the lake has spare capacity $\left(z * \mid 0<s_{t}<\right.$ $\bar{s})$;

(3) Optimal hydro generation when the lake is full $\left(z * \mid s_{t}=\bar{s}\right)$;

(4) Optimal gas generation when the lake is empty $\left(m * \mid s_{t}=0\right)$;

(5) Optimal gas generation when the lake has spare capacity $\left(m * \mid 0<s_{t}<\bar{s}\right)$; and

(6) Optimal gas generation when the lake is full $\left(m * \mid s_{t}=\bar{s}\right)$.

Diagrams 5-1 and 5-2 shows the optimal hydro and gas generation policy for a social planner when the lake is neither full nor empty. For the other 4 cases, see Appendix 3 for details. 


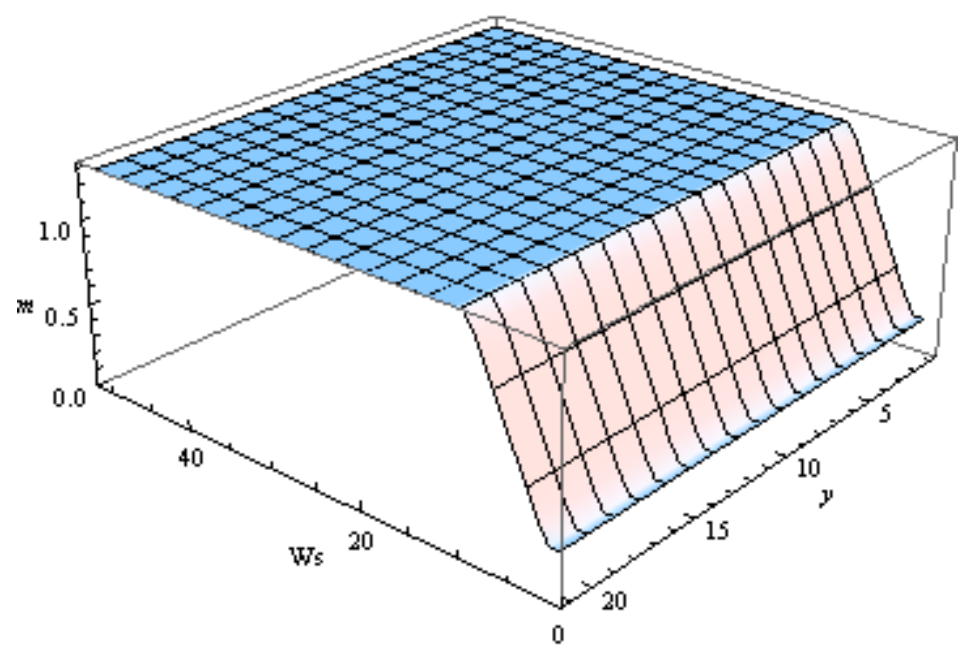

Diagram 5-1: Gas generation $\mathrm{m}^{*}$ for the social planner when the Lake is partially full $\left(0<s_{t}<\bar{s}\right)$

Diagram 5-1 shows the optimal gas generation policy of the social planner when the lake is neither empty nor full. When there is room for more storage, the optimal generation policy only depends on the shadow price of water $W_{s}$ : it is not a function of the inflow level. When the marginal value of stored water $W_{s}$ equals zero, the social planner chooses to generate 0.242896 units of electricity using the gas plant which is the optimal level of gas generation given that hydro plant is running at its full capacity (given that the shadow price of water equals zero). As the shadow price of water increases from zero the social planner will choose to substitute more gas for hydro, until gas becomes the only source of electricity generation, For all $W_{s}$ greater than this critical value, the amount of gas generation equals 1.29143 units. The minimum value of the shadow price $W_{s}$ in which the gas becomes the sole source of electricity generation is 2 (which is the $10^{\text {th }}$ value of $W_{s}$ in our grid). 


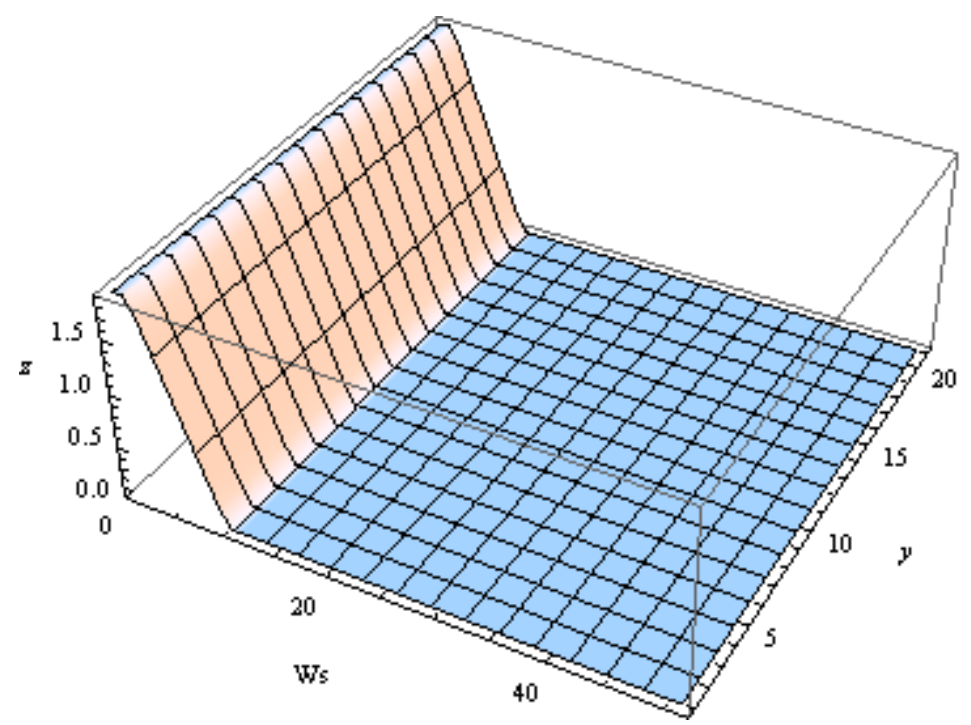

Diagram 5-2: Hydro generation $\mathrm{z}^{*}$ for the social planner when the lake is partially full $\left(0<s_{t}<\bar{s}\right)$

Diagram 5-2 shows the optimal hydro generation policy when there is water and spare storage capacity in the lake, that is, when the lake is neither empty nor full. At a point in time, there is water inflow from the river and stored water in the lake, thus no upper bound is imposed on hydro generation apart from capacity of the plant. As a result, the optimal hydro generation policy only depends on the shadow price of water $W_{s}$ : it is not a function of the inflow level. When the shadow price of water $W_{s}$ is very low at a value that is close to zero, we observe a high level of hydro generation that is capped at its maximum generation capacity $\bar{z}$. This is because when using the water is close to having no cost it is optimal for the social planner to generate electricity using the hydro plant as much as possible subject to the generation capacity constraint. However, as $W_{s}$ moves away from zero to higher values, the opportunity cost of using hydro resource in electricity generation increases. As a result it is optimal for the social planner to decrease the level of hydro generation $\left(z^{*} \mid 0<s_{t}<\bar{s}\right)$ as $W_{s}$ increases. This decline in $z^{*}$ will continue as $W_{s}$ increases until hydro generation reaches zero (when $W_{s}$ takes values higher than 2). 


\subsubsection{Determining the shadow price of water}

We use the policy iteration method (as described in section 3.1.3) to solve for the social planner's optimal generation policy in the dynamic setting on a discrete grid in $(s, y)$ space.

The grid is as follows: $s$ ranges from 0 to $0.17967{ }^{12}$ with 20 incremental steps and $y$ ranges from 0.2 to $3.5^{13}$ with 60 incremental steps. The initial generation policy is described by,

$$
\begin{gathered}
z^{(0)}(s, y)=-0.13537+0.088875+0.026331 y \\
m^{(0)}(s, y)=0.2 .
\end{gathered}
$$

Results converge within 12 iterations ${ }^{14}$. The results we obtained in the first 14 iterations for $\mathrm{s}=0.04492$ and $\mathrm{y}=1.85$ are summarized in the table below:

Table 5-1: Summary of iteration results.

\begin{tabular}{|l|c|c|c|c|}
\hline & $\begin{array}{c}\text { Hydro } \\
\mathrm{z}^{*} \mid\{\mathrm{s}=0.04492, \\
\mathrm{y}=1.85\}\end{array}$ & $\begin{array}{c}\text { Gas } \\
\mathrm{m}^{*} \mid\{\mathrm{s}=0.04492, \\
\mathrm{y}=1.85\}\end{array}$ & $\begin{array}{c}\text { Shadow Price } \\
\mathrm{Ws} \mid\{\mathrm{s}=0.04492, \\
\mathrm{y}=1.85\}\end{array}$ & $\begin{array}{c}\text { Electricity } \\
\text { Price } \\
\mathrm{P} \mid\{\mathrm{s}=0.04492, \\
\mathrm{y}=1.85\}\end{array}$ \\
\hline Starting & 1.29042 & 0.2 & 1.3915 & 1.58985 \\
\hline Iteration 1 & 0.446274 & 1.04528 & 1.10375 & 1.58786 \\
\hline Iteration 2 & 0.813052 & 0.842975 & 0.985672 & 1.30011 \\
\hline Iteration 3 & 0.963553 & 0.759964 & 0.915979 & 1.18203 \\
\hline Iteration 4 & 1.05238 & 0.710967 & 0.871596 & 1.11234 \\
\hline Iteration 5 & 1.10896 & 0.679764 & 0.87384 & 1.06796 \\
\hline
\end{tabular}

\footnotetext{
${ }^{9}$ Maximum capacity of the storage lake, for details see Section 4.3

${ }^{10}$ Given that the water inflow follows the processes described in Section 4.5, the water inflow level never falls below 0.3 and never exceeds 3.5 .

${ }^{14}$ Convergence occurs when $\mathrm{m}^{*}, \mathrm{z}^{*}, \mathrm{Ws}$ and $\mathrm{P}^{*}$ do not change to 6 decimal places. 


\begin{tabular}{|l|c|c|c|c|}
\hline Iteration 6 & 1.1061 & 0.681341 & 0.873964 & 1.0702 \\
\hline Iteration 7 & 1.10594 & 0.681428 & 0.873977 & 1.07032 \\
\hline Iteration 8 & 1.10592 & 0.681438 & 0.873983 & 1.07034 \\
\hline Iteration 9 & 1.10591 & 0.681442 & 0.873985 & 1.07034 \\
\hline Iteration 10 & 1.10591 & 0.681444 & 0.873986 & 1.07035 \\
\hline Iteration 11 & 1.10591 & 0.681444 & 0.873987 & 1.07035 \\
\hline Iteration 12 & 1.10591 & 0.681445 & 0.873987 & 1.07035 \\
\hline Iteration 13 & 1.10591 & 0.681445 & 0.873987 & 1.07035 \\
\hline Iteration 14 & 1.10591 & 0.681445 & 0.873987 & 1.07035 \\
\hline
\end{tabular}

Diagram 5-3 below shows the optimal storage policy of the New Zealand calibrated model after 14 iterations.

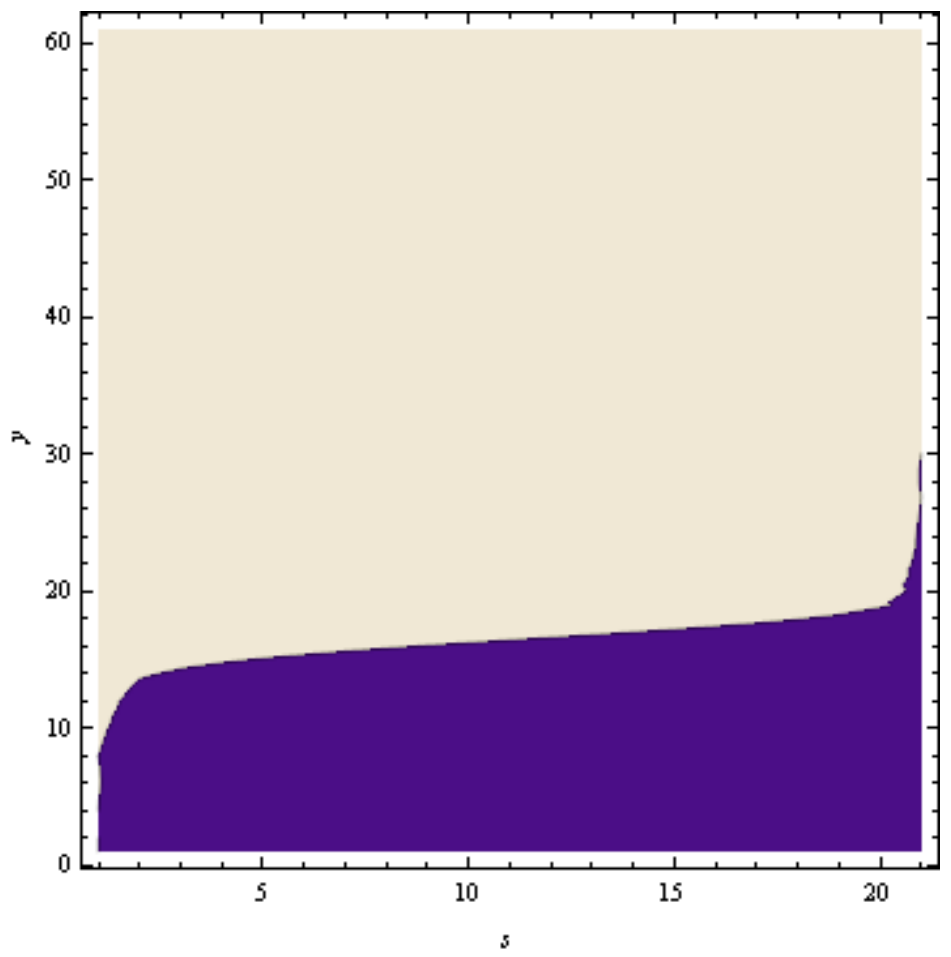

Diagram 5-3: Optimal storage policy in the space of storage and inflows $\{\mathrm{s}, \mathrm{y}\}$

For the combinations of $s$ and $y$ lying in the light shaded area, the amount of hydro generation is chosen to be less than the inflow from the river, so the storage level will go up; whereas the blue shaded area represents the 
combinations of $\mathrm{s}$ and $\mathrm{y}$ for which it is optimal for the social planner to lower the lake.

In the New Zealand calibrated competitive model, as long as the lake is not empty, for any storage lake level the social planner will be willing to lower the storage lake (and use it in electricity generation) given that the water inflow $y$ is lower than a certain level. The minimum level of water inflow required for the social planner to start filling up the storage lake increases as the amount of water that is already in the lake increases. That is, when the water inflow is high and there is enough spare room in the storage lake, it is more likely that the social planner will choose to put more water into storage. This is because the water arriving is so abundant that instead of using it all in generation at present date it is optimal to save it for electricity generation in a future period when the inflow is low, and storing it now for future use is feasible since the storage level is not at lake capacity. This describes a smoothing process that would shift generation fuel between time periods and smooth aggregate generation and prices across time and therefore increase the overall social benefit.

When the storage lake is full, the social planner can either withdraw water from the lake or maintain it at the current level. At any inflow level, the storage level cannot be increased where the lake has reached its maximum capacity.

On the other hand, if the level of water inflow at the present date is low and/or there is little space in the storage lake, it is more likely for the social planner to withdraw water from the lake and use it in generation at some date. As the lake level increases, the shadow price of water decreases. In other words, when the lake level is high, the opportunity cost of using water in the lake is relatively low. Given that the water inflow in this period is also low, the resulting market price if the generators only use water from the river for hydro generation would be 
higher than the opportunity cost of water in the storage lake. In such a case, it is socially optimal for the social planner to withdraw water from the storage lake and use it in electricity generation at that date.

The optimal choice of electricity generation varies with different combinations of storage level and water inflow. For each combination of storage level and water inflow, we are able to calculate the shadow price of water $W_{s}(s, y)$ using the finite difference method described in section 3. Given the shadow price of water $W_{s}$ and inflow level $y$, the optimal level of generation can be found using the optimal generation policies presented in section 5.2.1. Diagram 5-4 below shows the optimal hydro generation policy of New Zealand calibrated to the competitive model after 12 iterations and Diagram 5-5 presents the optimal gas generation policy.

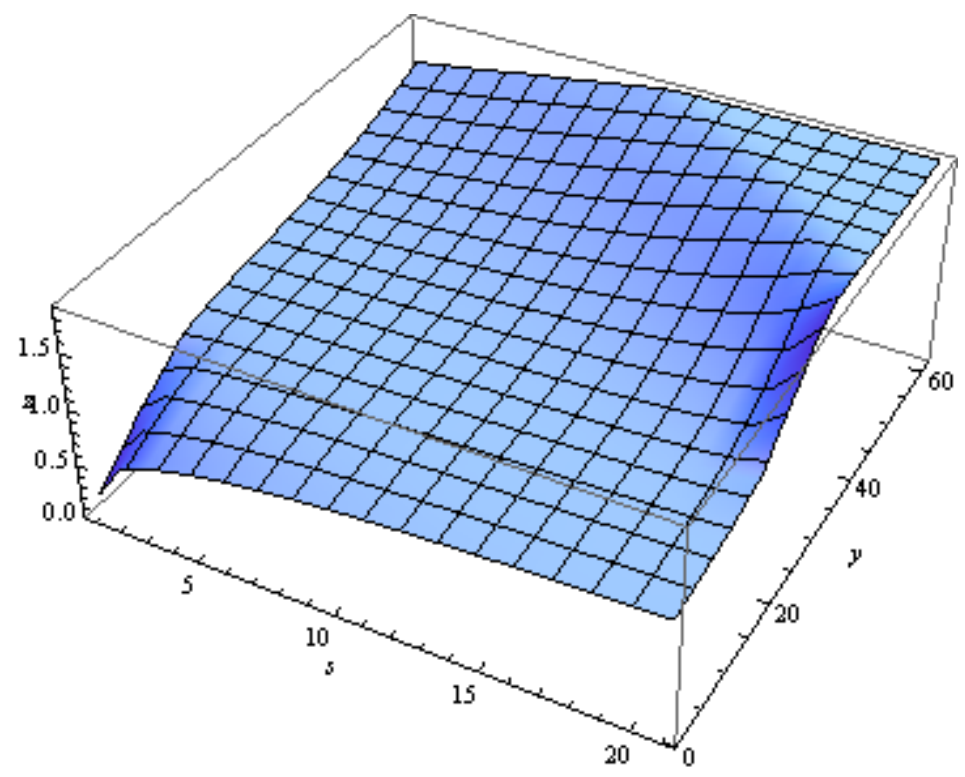

Diagram 5-4: Optimal hydro generation in storage and inflow dimensions $z^{*}(s, y)$ 


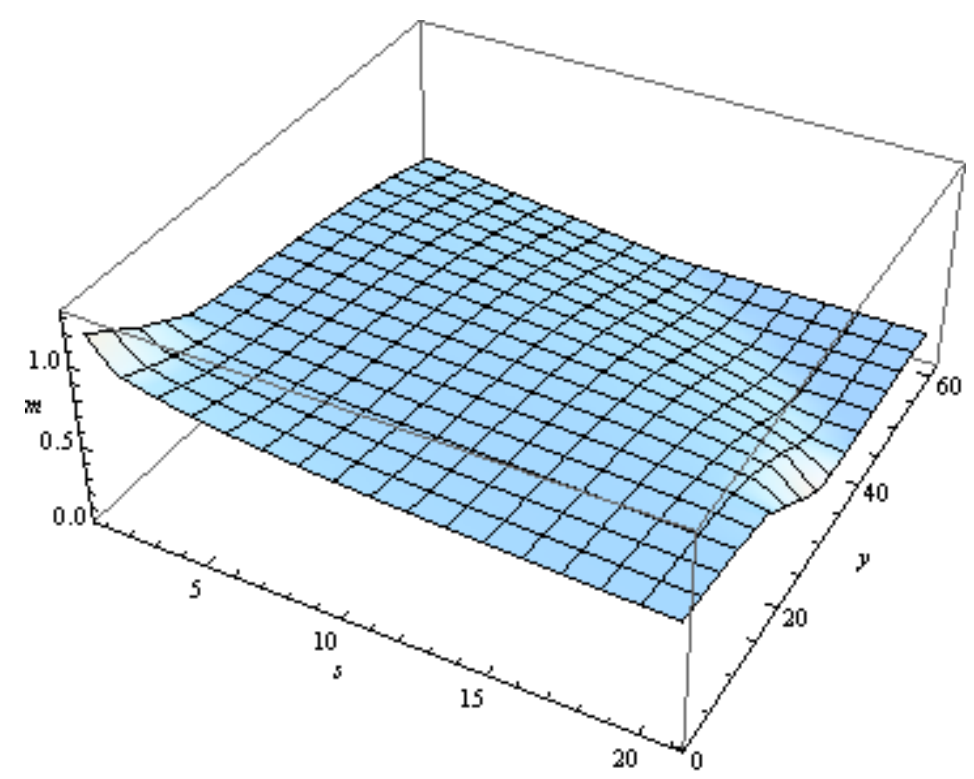

Diagram 5-5: Optimal gas generation in storage and inflow dimensions $m^{*}(s, y)$

We observe that hydro generation is low when storage and inflow are both low. The level of hydro generation increases as storage level and/or inflow level increases until it reaches its maximum generation capacity.

On the other hand, the gas generation serves as a substitute for hydro generation. The level of gas generation is the highest when the storage and inflow are both low, in other words when there is no hydro resource available. In this standard competitive model, we observe that the gas generation capacity in itself is large enough to satisfy the market demand even when there is no hydro resource at all. Being a substitute for hydro generation, gas generation decreases as the storage level and/or the water inflow increasea, and stops decreasing at a certain positive level ( $m=0.242896)$ when hydro reaches its generation capacity.

Diagram 5-6 below shows the shadow price of water as a function of storage lake level and inflow level. 


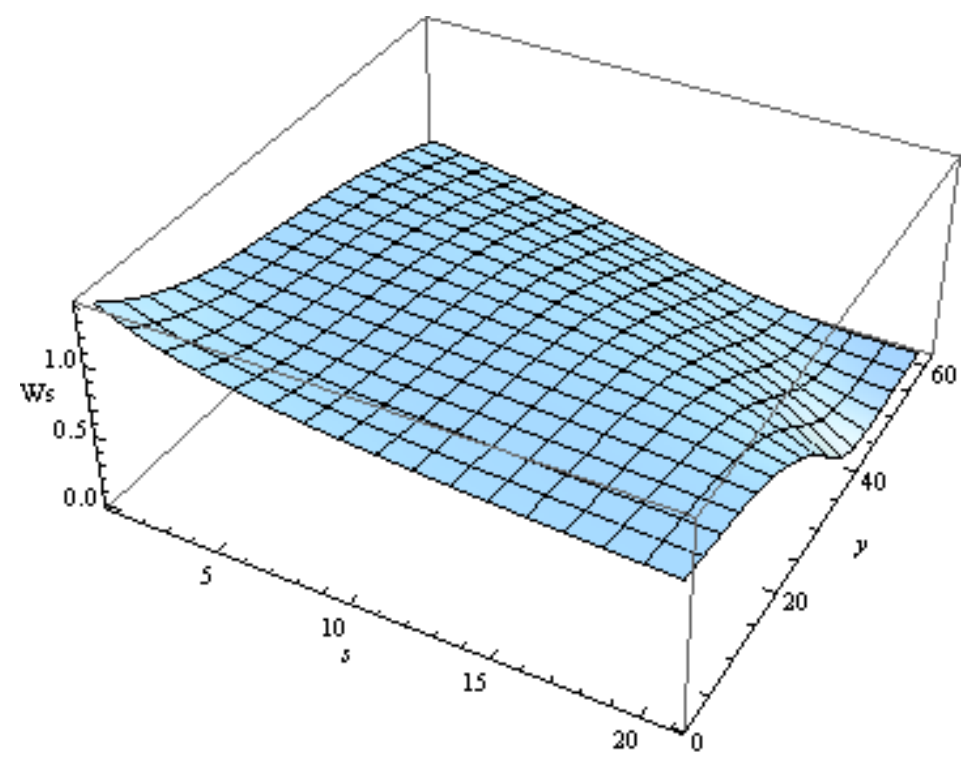

Diagram 5-6: Shadow price of water in storage and inflow dimensions $W_{s}(s, y)$

The shadow price of water is highest when the water inflow and the storage level are at zero.

When the lake level is low, the amount of hydro resource that is available to be used in future dry (low inflow) periods is very limited. It is in those dry periods that each additional unit of water available increases the total surplus by a greater amount. Therefore the value of stored water is high when the lake level is currently low. The shadow price of water would be even higher if the water inflow in that period is also low.

When the water inflow is low, only a small quantity of electricity can be generated by using the 'instant' water from the river. If we assume that the social planner will choose to withdraw one unit of water from the lake using it in generation, then it will increase the total surplus at the present date by a large amount (as the total quantity of electricity generated before this unit of electricity generated was small due to the low inflow). Therefore each unit of water in the storage lake represents a high value when the inflow is low. 
Also, when water inflow is low gas generation will be high and at a high marginal cost, thereby raising the value of a unit of hydro generation in this and perhaps in future periods.

On the other hand, when the inflow from the river is low, the water resource is so scarce that it is very likely that the social planner will not store any additional water. Given that the lake level is also low in that period, scarcity of the water resource in this period leads to a scarcity of the water resource in future periods (because the storage is low), which assigns a high value for each unit of stored water that is currently in the lake.

When the storage lake is close to empty, the value of an additional unit of water from inflows equals the value of an additional unit of water in the lake as it is most likely that they will both be used in generating electricity immediately.

As the water inflow increases and/or the lake level increases, the shadow price of stored water decreases since the water resource for electricity generation is more abundant both for now and for the future periods. The shadow price of water reaches its lowest value when the lake is full and the inflow is at its highest possible level. The shadow price of water reaches the value of zero when spilling take place which is when the lake is full and the amount of inflow exceeds the maximum generation capacity of the hydro plant.

Diagram 5-7 below shows the resulting market price in a perfectly competitive market as a function of lake level and inflow. 


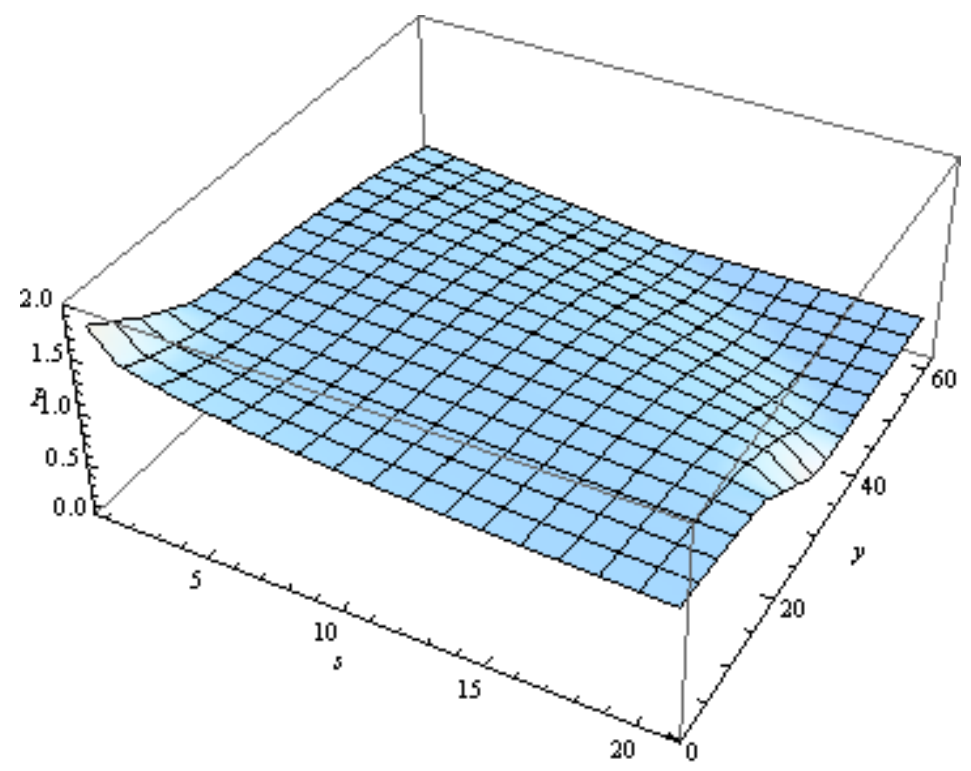

Diagram 5-7: Market price in storage and inflow dimensions $P(s, y)$

High price episodes tend to occur when both the inflow and the storage lake are low. In that region, the amount of water coming from the river is low and therefore the amount of water that can be used in generation (without lowering the storage lake) at the present time is limited. At the same time the shadow price of water being high when the storage level is low makes it too costly for the social planner to withdraw water from the storage lake and use it in electricity generation at that date. As a result, in equilibrium, the total quantity of electricity in the market includes only the quantity generated using the small amount of inflow available together with some quantity generated by the at the same time expensive gas. This small overall market quantity leads to a high price in the market.

However, as the water inflow level and storage level increase, the amount of instant water from the river increases and the opportunity cost of water in the storage decreases. As a result, a higher quantity of electricity will be generated using hydro plant at a lower cost which leads to a reduction in gas generation and an overall lower price in the market. 


\subsubsection{Simulation results}

Using the inflow calibration we have described, water inflow $y$ evolves according to the following square root process:

$$
d y_{t}=6.9448\left(1-y_{t}\right) d_{t}+0.9056 \sqrt{y_{t}} d \xi_{t}
$$

Assuming that at time zero the water inflow is at its long run mean $(\mu=1)$, we use the Monte Carlo simulation method to simulate a series of 30 years worth of daily water inflow data ${ }^{15}$, following the square root process stated above. The series of simulated inflow data is presented in Diagram 5-8.

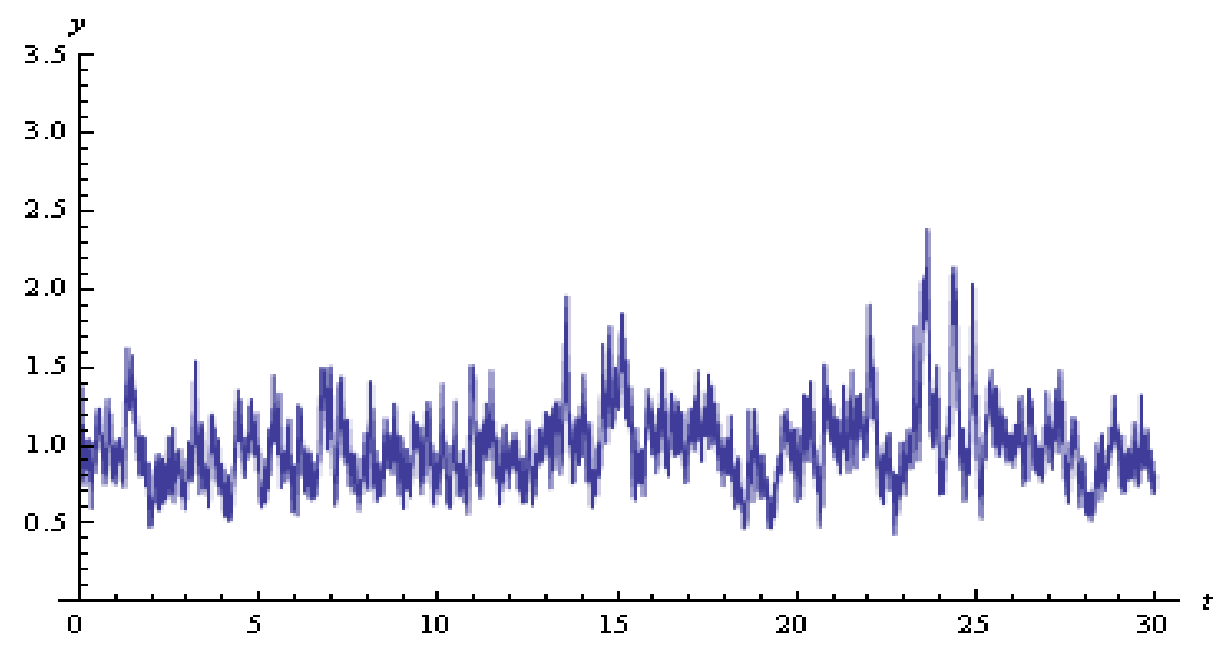

Diagram 5-8: Simulated water inflow $y$

${ }^{15}$ The equation for the Monte Carlo simulation is that

$$
\begin{aligned}
y_{t} & =y_{t-1}+\eta\left(\mu-y_{t-1}\right) d t+\sigma\left(y_{t-1} d t\right)^{\frac{1}{2}} \cdot \mathrm{N}(0,1) \\
& =y_{t-1}+6.9448\left(1-y_{t-1}\right) d t+0.9056\left(y_{t-1} d t\right)^{\frac{1}{2}} \cdot \mathrm{N}(0,1)
\end{aligned}
$$

where $\quad d t=1 / 365$. 
There are 10590 daily observations in total. These inflow data have a mean of 1 and a standard deviation of 0.243 . Most of the simulated inflow observations fluctuate between values of 0.5 and 1.5 except for some very dry periods; for example in August of the $23^{\text {rd }}$ year where the inflow reaches its lowest value in 30 years of simulation of 0.4 . There are also some high inflow episodes, for example from the mid of the $24^{\text {th }}$ year to the end of the $26^{\text {th }}$ year, with a high of 2.4 that occurs in June year 24 .

The reason for choosing to simulate 30 years worth of data is that it is long enough to include most of the high and low episodes of water inflow that are possible to occur in the long run, and enables a starting level of storage of zero to have negligible effect.

The distribution of inflow data is skewed in that there are more outliers on the up side than the downside. That is, the data suggest that it is more common for the market to have a large flood than to have a large drought. This matches with known properties of New Zealand water inflow.

We also assume that at time zero the storage lake is close to being empty but not at zero $^{16}$ : we set the initial lake level equal to 0.001 (the capacity is 0.17967 ).

Given the simulated 30 years of daily water inflow ( $y_{t}$ for $t$ from 1 to 10590) and initial values for storage $\left(S_{0}\right)$, the optimal choice for the social planner of the generation levels and storage level in each day can be found by using the optimal policies calculated for the social planner.

Diagram 5-9 presents the level of the optimal choice of storage over the 30 year window. The average storage level over 30 years is 0.0582519 which is about

\footnotetext{
${ }^{16}$ This is to prevent falling into boundary conditions for when the lake is empty.
} 
32.4 percent of the maximum lake capacity, with a standard deviation of 0.0496936 .

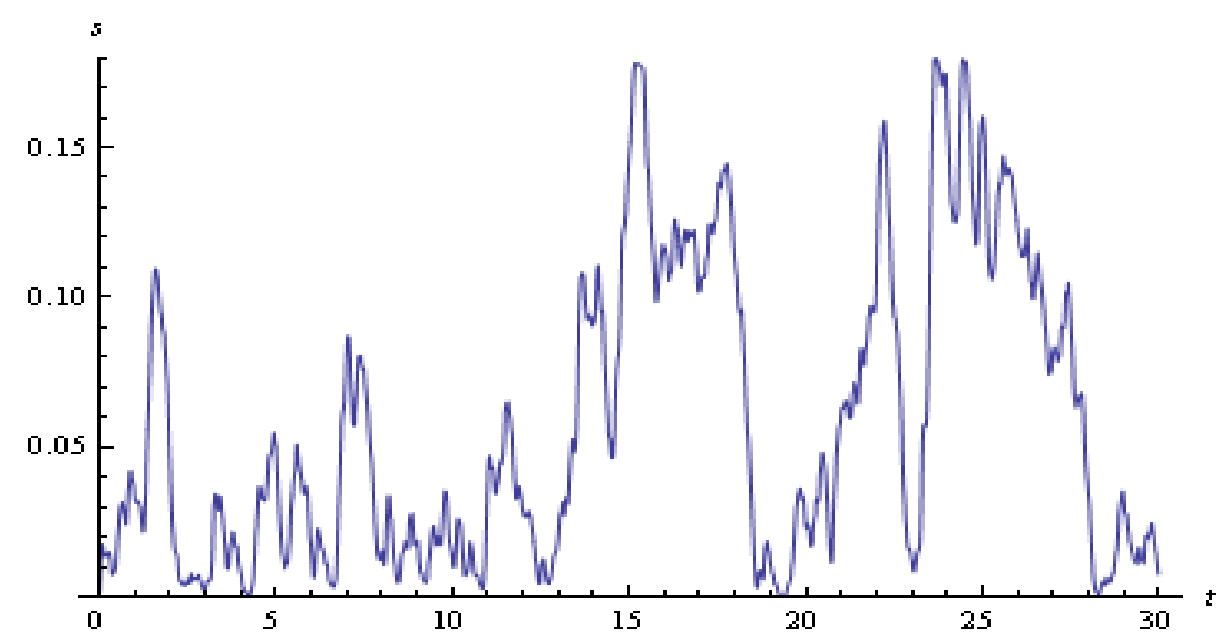

Diagram 5-9: storage lake level $s$

The social planner uses the storage lake on a frequent basis, and the pattern in the storage level positively responds to the fluctuations we observe in the simulated water inflow series with a correlation coefficient of 0.456861 . The planner puts more water into storage when the inflow is high, and withdraws from it when the inflow is low. The standard deviation of the lake level (0.0496936) is however considerably smaller than the standard deviation of the simulated inflow (0.242992). The fluctuation in water inflow process has been dampened as the inflow diversifies into its two different uses at a given date generation or storage.

Most of the time, the lake is not full; the lake level having been raised and lowered without reaching the two bounds. We see the lake being emptied several times within the 30 years time (for example, it is empty at the end of year 3 , the end of year 4, the end of year 11, the second quarter of year 20 and the first quarter of year 29). It also reaches its maximum storage capacity, at the start of year 16, middle of year 24 and the middle of year 25 . 
Emptying the lake involves a sustained period of withdrawal which happens when there is a sustained long period of low water inflow. As when low inflow occurs, it is still best for the social planner to withdraw water from the storage lake and use it in electricity generation. The time it takes to use up the water in the lake depends on the initial level of storage before the drought starts. For example at the start of year 5 and the end of year 11, the storage level has been quite low in the past one year (less than 0.03). However, because the storage was low initially, a half-year period of sustained below average water inflow is enough to bring the storage lake to empty. On the other hand, at the start of year 23 the storage lake is $89 \%$ full, one year of sustained below average water inflow brought it down to $5.5 \%$ as at the end of year 23 .

Similarly to have a full lake, requires a sustained level of high inflow. For example, in the first half of the $24^{\text {th }}$ year there was a half-year long period of sustained above average inflows, including some historically high inflow. This was enough to bring the lake from 5.5 percent full to its full capacity within 6 months despite hydro usage.

Diagram 5-10 presents the optimal level of daily hydro electricity generation in 30 years given the simulated inflow. The average daily hydro electricity generation is 0.986311 , which on average accounts for 56.9 percent of total electricity generation. 


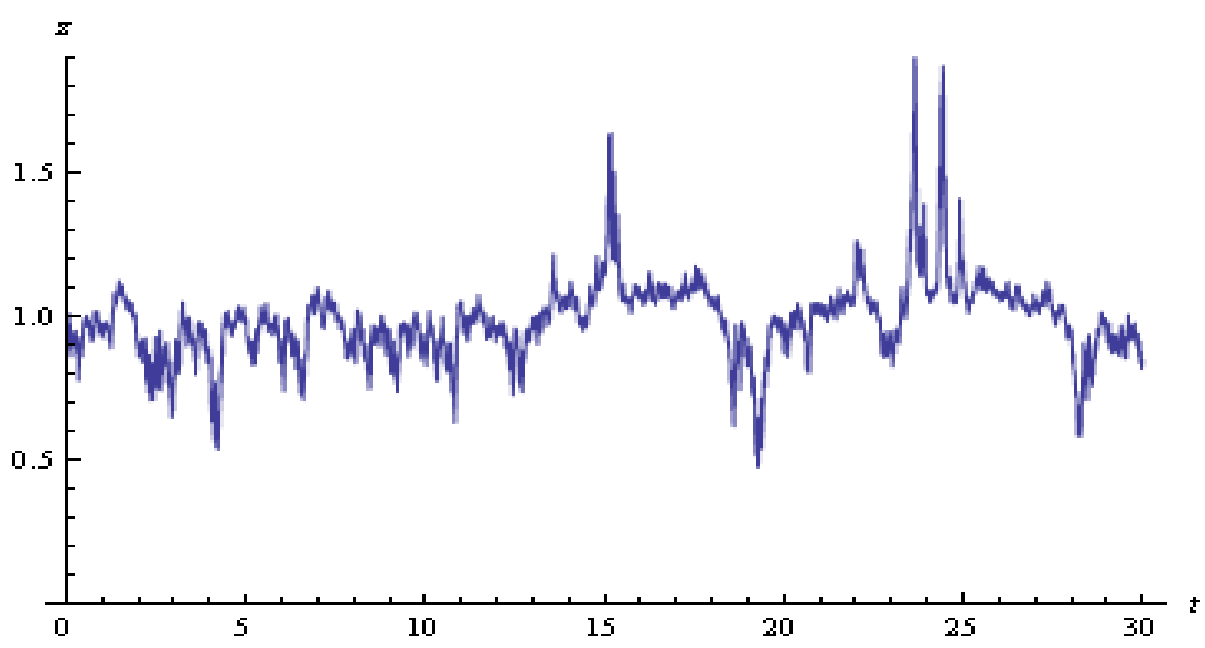

Diagram 5-10: Optimal hydro generation $z^{*}$

Hydro electricity generation follows the movements in the simulated water inflow, as the social planner tends to generate more using hydro when the inflow is high and cut back the hydro generation when the inflow is low. However, the magnitude of fluctuation in the hydro generation level is considerably smaller than that of the simulated water inflow, with a smaller standard deviation of 0.137723 comparing to a standard deviation of 0.242992 for the simulated inflow. This is because part of the volatility has been smoothed by the use of storage. When the inflow is low, the social planner tends to have low hydro generation. However, the amount of hydro generation is not limited to the amount coming from the inflow as the social planner can withdraw water from the storage which was saved from the past high inflow period. On the other hand, when the inflow is high the social planner uses more water in generation but not the entire amount of inflow; instead they put some into the storage for use in future dry periods.

However, the storage facility's ability to smooth the hydro generation over time is restricted by its capacity constraints. When the storage level is low, the storage lake is able to smooth the hydro generation by removing the high spikes in the inflow process by putting plentiful hydro resources into storage. Nevertheless while the storage is low, it is unable to respond to a low inflow shock, as there is 
not enough water in storage. This statement is confirmed by the behaviour of the first 10 years of observations. The simulated water inflow process fluctuates a lot within the range 0.5 and 1.5 in the first 10 years. The storage level stays at a low level most of the time. Comparing the hydro generation process with the simulated inflow process, the hydro generation process is smoother than the inflow process with most of the fluctuations staying within the range 0.5 and 1.0. Most of the remaining volatility corresponds to the low inflow shocks, particularly when the storage is empty, in particular at the start of year 5 and at the end of year 11. On the other hand, when the storage level is close to being full, it is less able to smooth the high inflow shocks as storage has little scope to store more water. Therefore when the storage is close to being full, while the hydro generation is smoother on the downside, any high inflow shock will also lead to a spike in hydro generation level. This explains the three abnormally high spikes we observed in hydro generation process at the start of year 16, middle of year 24 and middle of year 25

Hydro generation reaches its maximum capacity for two weeks in the middle of the $24^{\text {th }}$ year. Before this occurs there had been a sustained long period of high inflows which gradually increased the storage level. With continuing high inflows, and high storage levels, hydro generation reaches its maximum capacity with excess water allocated to storage. Once the maximum storage level is reached, the residual inflow after generating hydro electricity at maximum capacity is spilled. This is the only occasion when spilling occurs. This can be more clearly observed in the following three diagrams which zoom in to take a close look at the 36 observations for hydro generation, storage and the difference between inflow and hydro generation (inflow net of hydro) around the time when spilling occurs. 


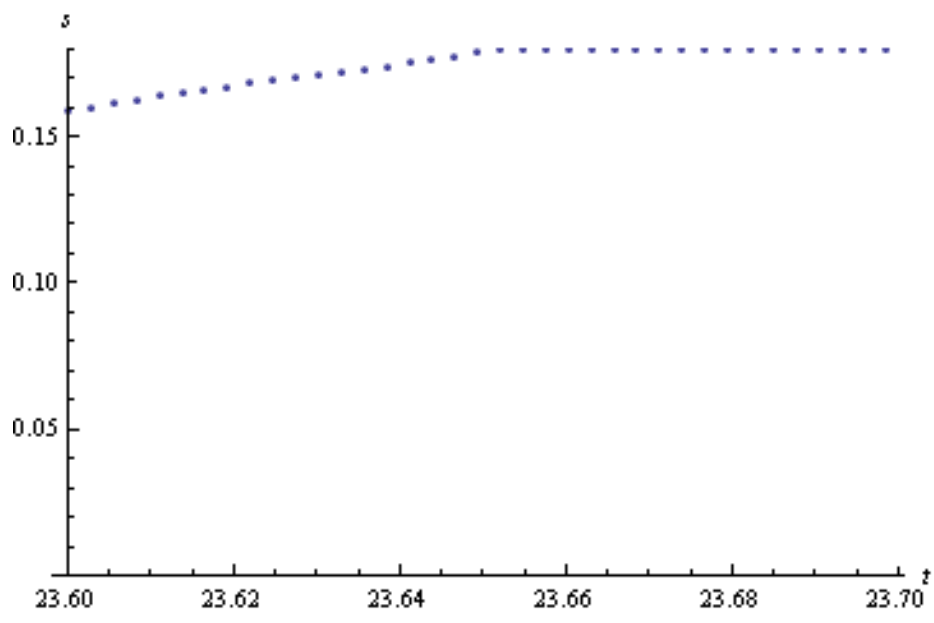

Diagram 5-11: Storage (for $\mathrm{t}$ from 23.6 to 23.7 )

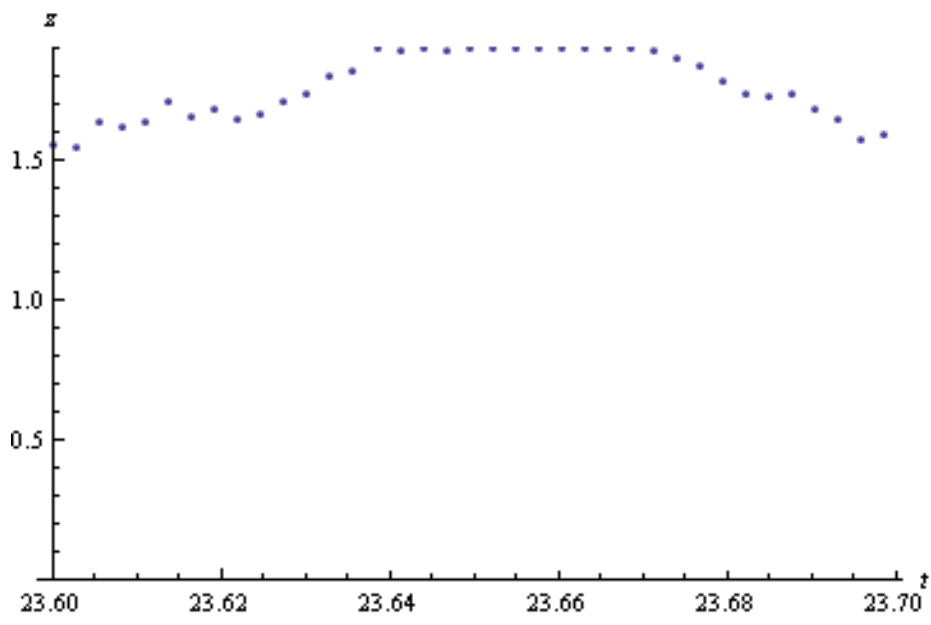

Diagram 5-12: Hydro generation (for $t$ from 23.6 to 23.7)

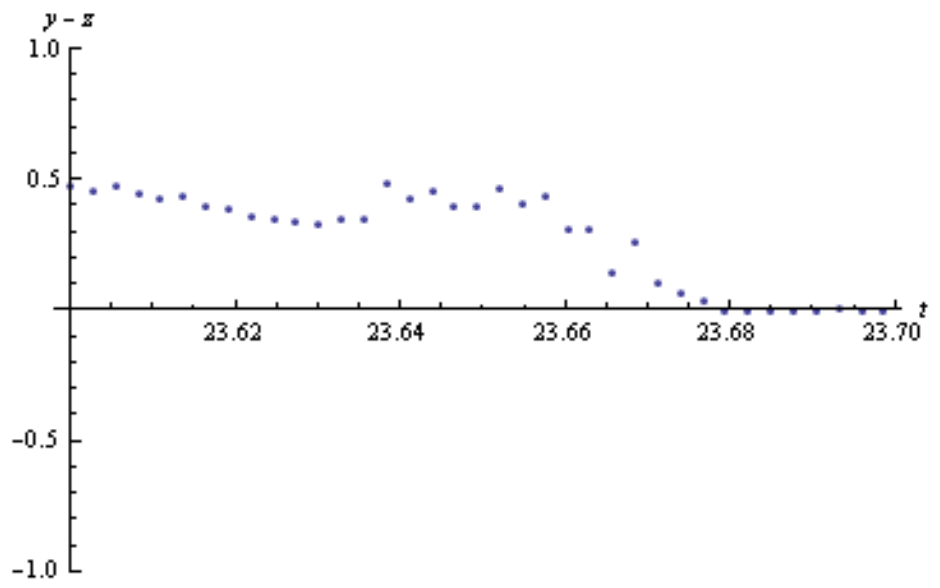

Diagram 5-13: Inflow net of hydro generation (for $t$ from 23.6 to 23.7)

Diagram 5-14 presents the optimal level of gas generation over the 30 years given the simulated water inflow. The average daily gas generation is 0.747411 , 
which on average accounts for 43.1 percent of total electricity generation. Compared to the hydro generation, gas generation has a smaller volatility with standard deviation of 0.0759636 .

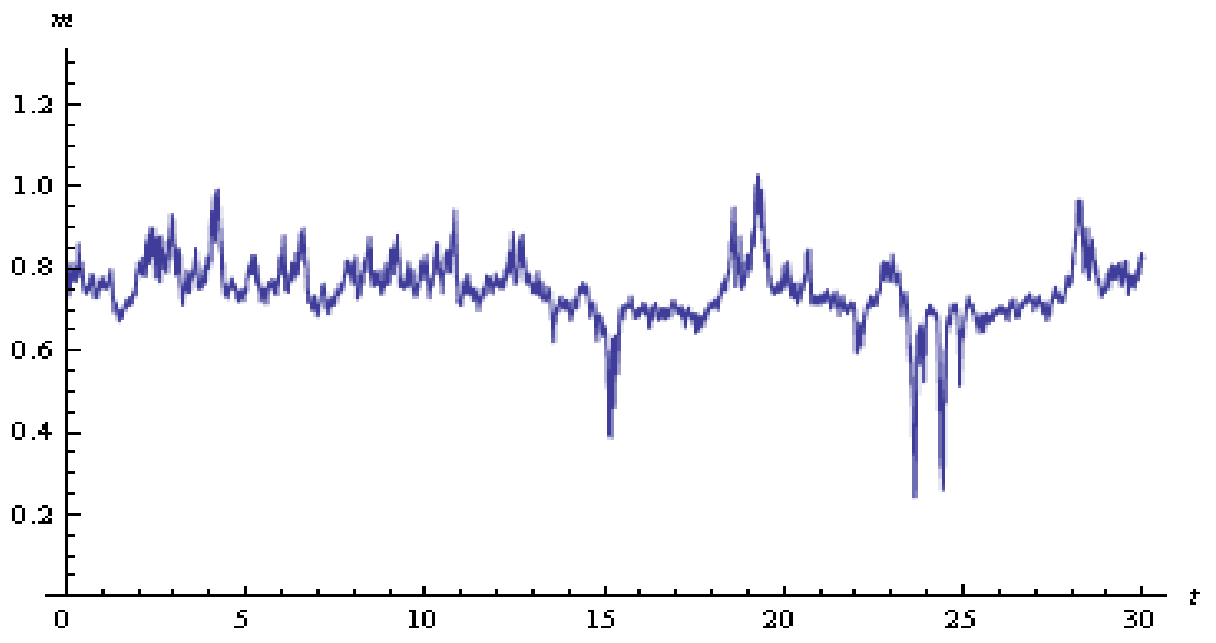

Diagram 5-14: Optimal gas generation $m^{*}$

We observe that in the 30 years of daily observations, gas has been used as a substitute for hydro generation with a correlation coefficient between the two sources of generation of negative one. The reason for gas being a perfect substitute for hydro generation is that certain model features have guaranteed gas generation being at an interior solution - the low transmission cost of gas guarantees a positive level of gas generation while the generation capacity of the gas plant is large enough to avoid a maximum gas generation capacity. Given that the marginal cost function of the gas plant is linear, being in an interior solution all the time means that we are always moving up and down the marginal cost of gas generated electricity. As a result, gas always serves as a perfect substitute for hydro generation as long as the gas plant does not reach its boundary ${ }^{17}$. However, though the correlation coefficient equals negative one, the rate of substitution between the two sources of generation is not one-for-one,

\footnotetext{
17 This perfect substitution between the two generations breaks down when the gas generation capacity is small.
} 
that is why we observe fluctuations in the market quantity and price, which is observable in Diagram 5-15 below, instead of being constant over time.

At each point in time, the amount of hydro generation and the amount of gas generation sum up to the total quantity generated in the electricity wholesale market. The market price coincides with the intersection of the market demand curve and this given aggregate quantity.

Diagram 5-15 presents the market price in the 30 year period.

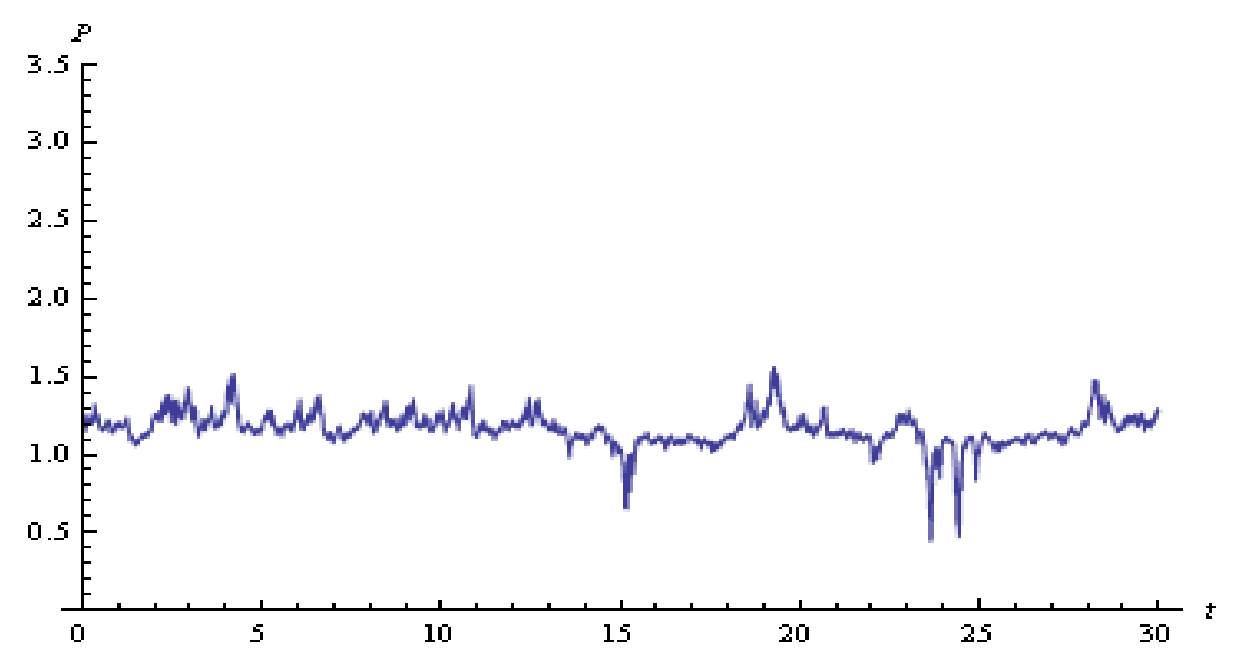

Diagram 5-15: Market electricity price $P^{*}$

The average market price over the 30 years is 1.16418 . The standard deviation of the market price ${ }^{18}$, which is equal to 0.108051 , is lower than the standard deviation of the hydro generation, but higher than the standard deviation of the gas generation.

The standard deviation of the price is considerably smaller than the standard deviation of the simulated water inflow and it is also smaller than the standard deviation of the hydro generation. This is because the storage enables smoothing

${ }^{18}$ The standard deviation of market price does not equal the standard deviation of the market quantity, as the coefficient on quantity in the inverse demand function does not equal negative one. 
in hydro generation across time which first removes part of the volatility in the water inflow and left the hydro generation with a smaller standard deviation relative to the volatility in inflow. Then the gas generation further smoothes the total quantity by substituting with hydro generation in each given period; the extent depending upon the state of storage and inflows.

The price process is generally stable except for three significant price falls which occur at the start of year 16, mid of year 24 and 25. These large price drops correspond to the high spikes we observe in the hydro generation process, which occurs when there is a high inflow while the storage is full. The abnormally high hydro generation leads to a high overall generation and a low market price.

Though the shadow price of water is not directly observable to the market, we are able to calculate its value using our model. Diagram 5-16 presents the value of the shadow price of water over the 30 year period.

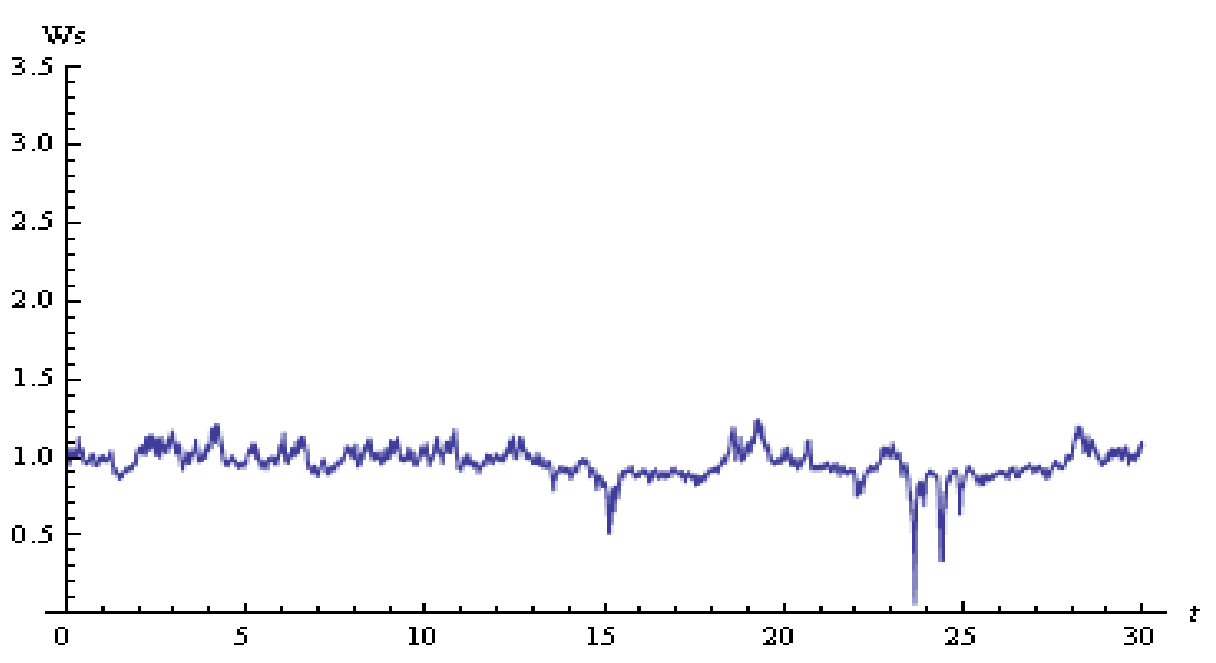

Diagram 5-16: Shadow price of water $W s$

The value of the shadow price of water over the thirty years has an average of 0.964722 , with a standard deviation of 0.0981244 . 
The shadow price of water is negatively correlated with the storage lake level with a correlation coefficient of -0.830091 , and is also negatively correlated with the inflow level with a correlation coefficient of -0.790925 . The value of each additional unit of stored water is low when the water resource is abundant which include both the amount in the storage and the amount from the inflow. However, the size of the correlation coefficients suggests that the shadow price of water reacts to a change in the storage lake level slightly more aggressively than it reacts to a change in the inflow level (both negatively) while holding other variables constant.

On the other hand, the shadow price of water is closely and positively correlated with the market price with a correlation coefficient of 0.985153 . In general the market price equals the shadow price of water at that time plus the cost of transmitting one unit of hydro generated electricity, except at times when storage is either empty or full. When the lake is empty, the social planner loses its ability to use more hydro resource in electricity generation other than the amount he has extracted from the inflow in that period. If the inflow in that period is small, in equilibrium the social planner would like to use more hydro resource in generation than what the inflow provides. However, since the storage is empty, in equilibrium the social planner will choose to put all inflow into generation and the resulting equilibrium price exceeds the summation of the shadow price of water and the transmission cost of hydro generated electricity. On the other hand, when the storage lake has reached its full capacity, it loses its ability to store more water. Since no additional water can be stored for future use, the opportunity cost of water is positive only for the amount of water stored in the lake. There is no opportunity cost of using water coming from the water inflow as other than being used in generation they will have to be spilled. As a result, we observe big drops in the shadow price of water in periods where the storage is full, for example at the start of year 16, the mid of year 24 and 25 . The drop was particularly sharp in the mid of year 24 , as 
that is when the amount of water inflow exceeded the hydro generation capacity, and excess water had to be spilled which bought the shadow price of water down to a very low number.

\subsection{Results of the monopoly model}

The approach to solving the monopolist's problem is similar to the social planner's except instead of maximizing social welfare, the monopolist aims at maximizing its profit. His objective function is therefore the following,

$$
\max _{m, z} f=\varphi(m+z) \cdot(m+z)-z \frac{\partial W}{\partial s}-C(m)-k_{1} z-k_{2} m .
$$

Since the static optimization results are largely qualitatively similar to the social planner's, we will skip those and jump straight to the simulation results. The diagrams of the static optimization can be found in Appendix 6.

\subsubsection{Simulation results}

To study the outcomes in a monopoly electricity market and ensure that the environment we impose in this market is consistent with the one we imposed in the competitive market (in order to conduct valid comparison between the outcomes of those two models), we use the same series of simulated inflow data as the one we used for the standard competitive model in section 5.2.3. The series of simulated inflow data is presented in Diagram 5-8.

Given the simulated 30 years of daily water inflow ( $y_{t}$ for $t$ from 0 to 10590) and initial values for storage $\left(s_{0}\right)$, the profit-maximizing choice for the monopolist generator of the generation levels and storage level in each day can be found by using the optimal policies calculated in section 5.3.2. 
Diagram 5-17 presents the level of the optimal storage over the 30 year window. The average storage level over 30 years is 0.0887507 which is about 49 percent of the maximum lake capacity, with a standard deviation of 0.059552 .

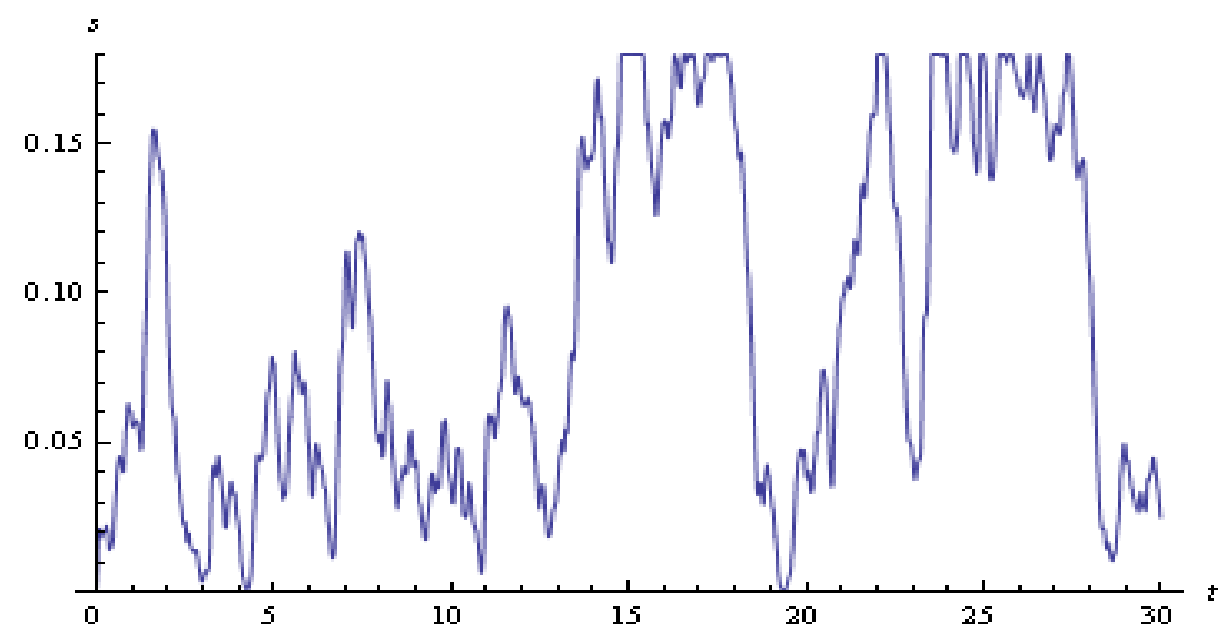

Diagram 5-17: Storage lake level $s$ in a monopoly market

The monopolist generator uses the storage lake on a very frequent basis, and the pattern in the storage level positively responds to the fluctuations we observe in the simulated water inflow series with a correlation of 0.40272 . The fluctuation in water inflow has been dampened as the inflow diversifies into its two different uses at a given date - being used in generation or being stored. The storage serves to reallocate the water resources across time. As a result, we observe that the standard deviation of the lake levels (0.0595522) is considerably smaller than the standard deviation of the simulated inflow (0.242992).

The lake reaches its maximum capacity for decent periods of time, especially during the periods of high inflows for example in periods between year 15 and year 18, and in periods between year 24 and 28. Also during those periods, it tends to keep the lake full for quite a while before it starts withdrawing water from it. The lake was kept at full capacity for close to a year (in year 14 and15) before being drawn down. 
In other periods we observe frequent actions being taken: raising and lowering the lake. In general, compared to the storage level in the competitive model, the monopoly generator tends to have a higher level of storage. At a given level of high inflow, the monopoly generator in general puts more water into storage relative to what the social planner in a competitive market setting would have done. This is reflected in the fact that the mean of 30 years of storage levels in the monopoly market is higher than the mean of storage levels in the competitive market (mean of storage level equals 0.0887507 in the monopoly market and equals 0.0582519 in the competitive market). This is because to achieve a higher profit, the monopoly generator has an incentive to keep the hydro generation level and the overall electricity generation level low to maintain the market price at a high level.

In fact, the monopoly generator never completely empties the lake in the 30 years. Though the storage level in periods around the end of year 4 and the second quarter of year 19 seem to be quite close to zero, they never actually reach zero. This can be seen more clearly in the following Diagram 5-18 which zooms in to take a closer look at the observations for lake level at values between 0 and 0.01 .

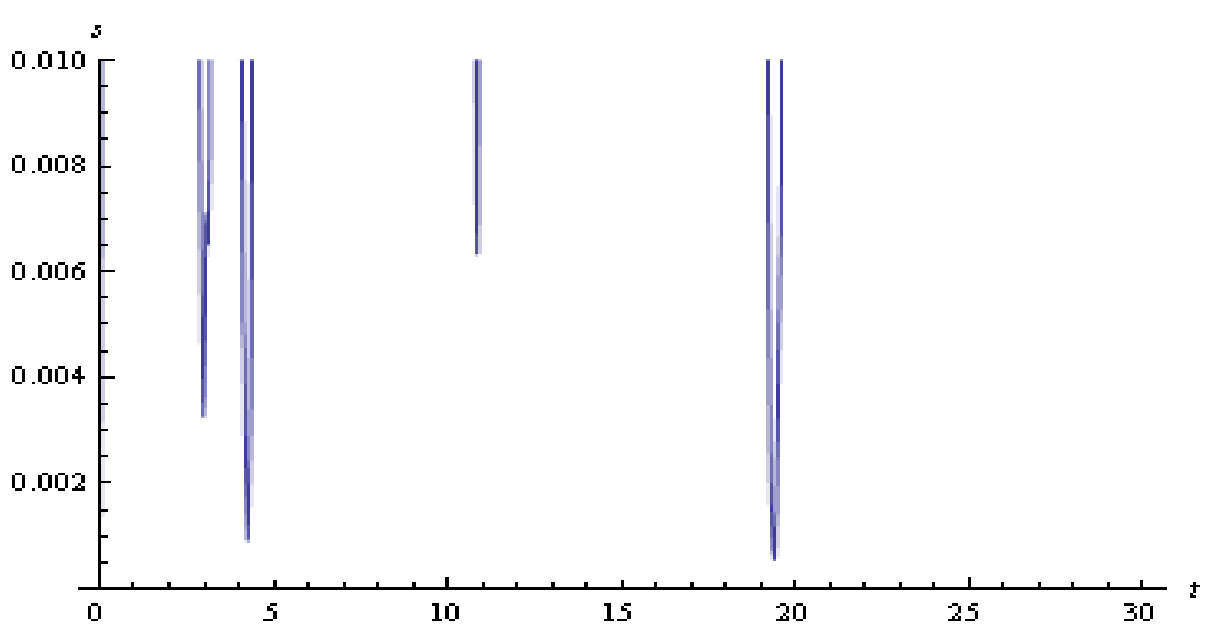

Diagram 5-18: Storage levels in a monopoly market that have values between 0 and 0.01 
The blue curve in Diagram 5-19 presents the optimal level of daily hydro electricity generation in 30 years given the simulated inflow. The average daily hydro electricity generation is 0.962985 , which on average accounts for $86.6 \%$ percent of total electricity generation.

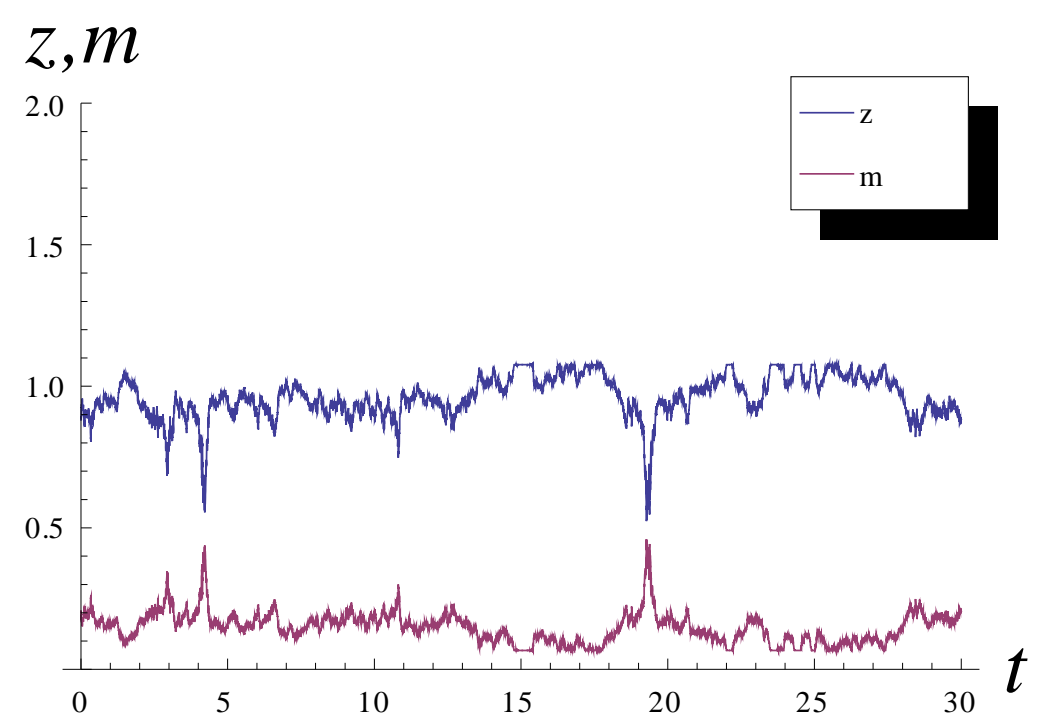

Diagram 5-19: Optimal hydro generation $z *$ in a monopoly market

Hydro electricity generation follows the movements in the simulated water inflow, more on the down side rather than on the up side. The monopoly generator tends to cut back the hydro generation when the inflow is low whereas when the inflow is high the generator increases the amount of hydro generation until its unconstrained optimal level of hydro generation is achieved (at 1.0821 units), and the remaining inflow is placed into the storage if there is spare capacity in the lake, otherwise it is spilled. The use of storage and spilling assigns the hydro generation level a much smaller volatility relative to of the simulated water inflow, with a standard deviation of 0.0753554 comparing to a standard deviation of 0.242992 for the simulated inflow. 
The two big drops in the hydro generation levels occur in periods from the fourth quarter of year 4 till the first quarter of year 5, and in periods in year 20 and both took place when there was a sustained period of low inflows along with a low lake level. For example during these low inflow periods in the end of year 4 and beginning of year 5 (which lasted for approximately 135 days), the monopoly generator not only uses all the inflow in hydro generation in each period, but also withdraws water gradually over time to contribute towards hydro generation. This gradual lowering of the lake causes the lake to reach a level that is very close, but not equal to, zero (approximately 0.001). At this point, the hydro generation is at one of its lowest levels in 30 years, at 0.54 units. After that the inflow level increases. However, we expect to see that if the inflow remains at low levels for even a small number of periods, the lake level will soon be completely depleted and there will be an even sharper drop in hydro generation.

Hydro generation reaches its unconstrained optimal level quite often in periods in years from 15 to 18 and from 24 to 28 . In these periods, in general both the inflows and the storage levels are high. The hydro resources from both of the sources above provide the monopoly generator with an amount of water that is more than he wants and essentially removes the hydro resources constraint. Spilling occurs when the inflow exceeds the amount of water required for generating the unconstrained optimal level of hydro generation $(y>1.0821)$ while the lake is full. This is a voluntary spilling as instead of spilling the water the monopoly generator could use it in hydro generation as the generation capacity of the hydro plant has not been reached. However, the monopolist generator chooses to generate at the unconstrained optimal level which is below the maximum capacity to make sure the price does not go too low, as this maximizes his profit. 
The purple curve in Diagram 5-19 presents the optimal level of gas generation over the 30 years given the simulated water inflow. The average daily gas generation is 0.147672 , which on average accounts for only 13.7 percent of total electricity generation. Compared to hydro generation, gas generation has a smaller volatility with a standard deviation of 0.0535763 .

We observe that in the 30 years of daily observations, gas has been used as a substitute for hydro generation with a correlation coefficient between the two sources of generation equal to negative one. The reason for gas being a perfect substitute for hydro generation all the time is that certain model features have guaranteed gas generation being at an interior solution - the low transmission cost of gas guarantees a positive level of gas generation while the generation capacity of the gas plant is large enough to avoid a maximum gas generation capacity. Given that the marginal cost function of the gas plant is linear, being in an interior solution all the time means that we are always moving up and down the marginal cost of gas generated electricity. As a result, gas always serves as a perfect substitute for hydro generation as long as the gas plant does not reach its boundary. However, though the correlation coefficient between hydro generation and gas generation is negative one, the substitution between the two sources of generation is not one-for-one, that is why we observe fluctuations in the market quantity and price, which is observable in Diagram 5-19, instead of being constant over time.

Diagram 5-20 presents the market price in the 30 year period. 


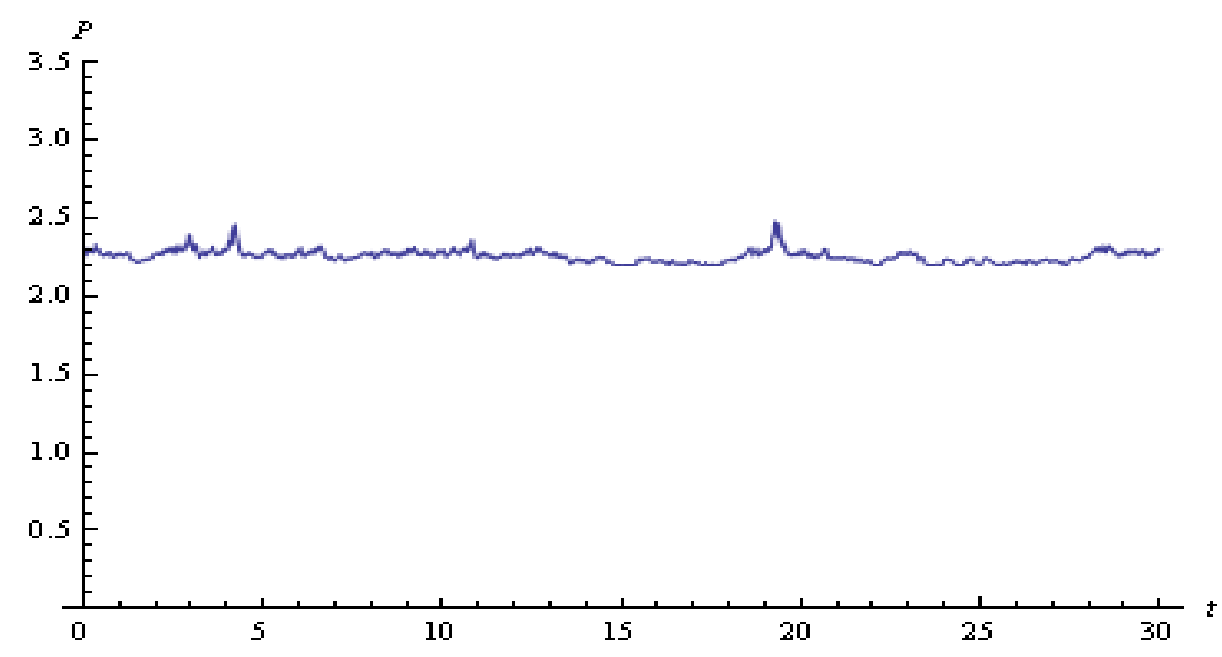

Diagram 5-20: Market electricity price $P^{*}$ in the monopoly market

The average market price over the 30 years in the New Zealand calibrated monopoly market is 2.25426 . The volatility of the market price is smaller than the volatility of hydro generation and gas generation, with standard deviation of 0.0381036. The reason for having a smaller volatility deviation is that the difference between the standard deviations of the two sources of generation is relative smaller (with standard deviation for hydro equal to 0.0753554, and standard deviation for gas equal to 0.0535763$)$. Most of the volatility in hydro generation and in gas generation cancels out as one substitutes for the other which brings an overall lower volatility for the market quantity and market price. 19

The standard deviation of the price is much smaller than the standard deviation of the simulated water inflow and it is also smaller than the standard deviation

\footnotetext{
${ }^{19}$ Given that the demand is linear expressed in form of $P(m+z)=a+b(m+z)$, $\operatorname{Var}(P)=b^{2} \operatorname{Var}(m+z)=b^{2}[\operatorname{Var}(m)+\operatorname{Var}(z)+2 \operatorname{Cov}(m, z)]$$$
=b^{2}[\operatorname{Var}(m)+\operatorname{Var}(z)+2 \rho(m, z) \sqrt{\operatorname{Var}(m)} \sqrt{\operatorname{Var}(z)}
$$

, and as the correlation coefficient between $m$ and $z(\rho(z, m))$ equals -1 ,

$\operatorname{Var}(P)=b^{2}[\operatorname{Var}(m)+\operatorname{Var}(z)-2 \sqrt{\operatorname{Var}(m) \operatorname{Var}(z)} ;$

If $\operatorname{Var}(m)=\operatorname{Var}(z)$,

$\operatorname{Var}(P)=b^{2}[\operatorname{Var}(m)+\operatorname{Var}(z)-2 \sqrt{\operatorname{Var}(m) \operatorname{Var}(m)}=0$.
}

That is, the closer the value of $\operatorname{Var}(m)$ and $\operatorname{Var}(z)$ are to each other, the smaller the $\operatorname{Var}(P)$; when they equal, $\operatorname{Var}(P)=0$. 
of the hydro generation. The gas generation further smoothes the total quantity by substituting with hydro generation in each given period which brings a lower standard deviation to prices.

In this price series, there are a number of high spikes which correspond to periods with low storage and low inflow. However, there is no significant price falls, as the monopoly uses the storage to control the level of hydro generation when inflow is high in order to ensure the price does not fall below 2.196887 (which is the price at when hydro generation equals its un-constrained optimal level, $z=1.07645)$.

Though the shadow price of water is not directly observable to the market, we are able to calculate its value using our model. Diagram 5-21 presents the value of the shadow price of water in the monopoly model over the 30 years period.

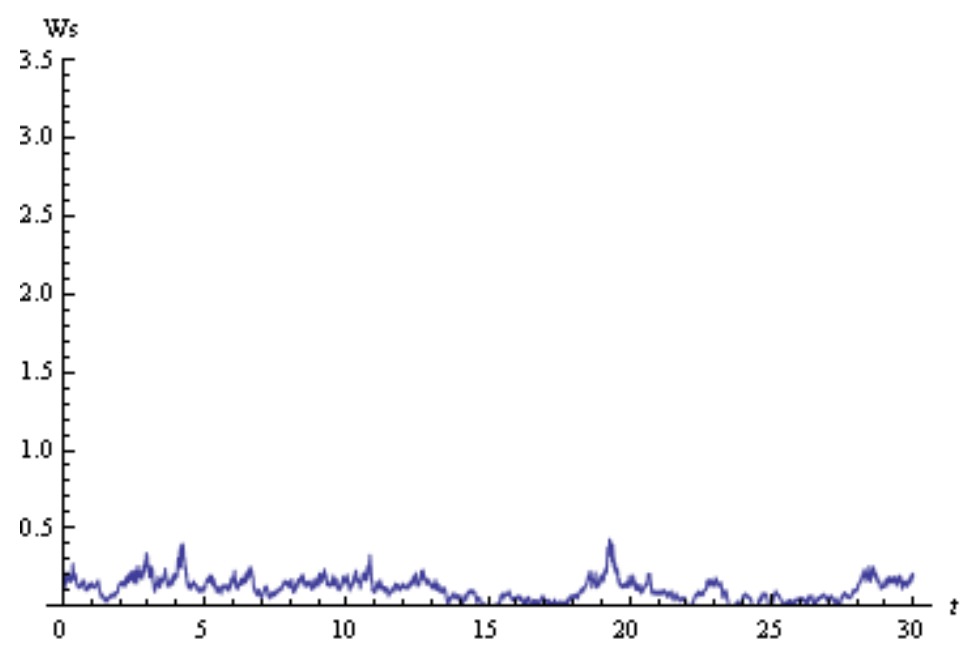

Diagram 5-21: Shadow price of water $W_{S}$

The shadow price of water over the thirty years has an average level of 0.11452 , with a standard deviation of 0.0690524 .

The shadow price of water is negatively correlated with the storage lake level with a correlation coefficient of -0.891693 , and is also negatively correlated with 
the inflow level with a correlation coefficient of -0.658662 . The value of each additional unit of stored water is low when the water resource is abundant which include both the amount in the storage and the amount from the inflow. However, the size of the correlation coefficients suggest that the shadow price of water reacts to a change in the storage lake level more than it reacts to a change in the inflow level (both negatively) while holding other variables constant.

On the other hand, the shadow price of water is closely and positively correlated with the market price with a correlation coefficient of 0.987728 . Most of the time, the summation of the shadow price of water at that time and the cost of transmitting one unit of hydro generated electricity equals the marginal revenue earned by the monopoly generator at the given quantity (which is smaller than the price at that quantity), except at times when the storage lake is full. ${ }^{20}$ When the lake reaches its full storage capacity, it loses its ability to store more water. Since no additional water can be stored for future use, the opportunity cost of water is positive only for the amount of water stored in the lake. There is no opportunity cost of using water from the inflow as other than being used in generation it will have to be spilled. As a result, we observe low values of shadow price of water in periods where the storage is full, for example in some periods from year 15 to year 18, and from year 24 to 28. During these periods, if the inflow is greater than the amount needed to generate the unconstrained optimal level of hydro generation, the corresponding shadow price of water equals zero and that is when spilling takes place.

\footnotetext{
${ }^{20}$ We do not need to consider the case when the lake is empty in this monopoly model, as in this model the generator never empties the lake. For details see Diagram 5-33.
} 


\subsection{Comparison between the competitive model and the monopoly model}

In this section, we compare the results from the standard competitive model and from the standard monopoly model. In part one, we look at the difference between the price behaviours and generation policies of a competitive market and those of the monopoly market; In part two, we look at the differences in their storage policies and the value of the storage lake; In the last part, we will discuss the differences in their resulting welfare consequences.

\subsubsection{Market outcomes}

Given the simulated series of 30-year of daily inflow data (as presented in Diagram 5-12), we calculated the optimal level of generation and the market equilibrium price in each period, first in a competitive market and then in a monopoly market. Table 5-2 below summaries the statistical information, which includes mean and standard deviation, on price, hydro-generation and gas generation of the competitive model and of the monopoly model.

\begin{tabular}{|c|c|c|c|c|}
\hline \multicolumn{2}{|c|}{ Table5-2 : Competition and monopoly market outcomes compared } \\
\hline Quantity & \multicolumn{2}{|c|}{ Mean } & \multicolumn{2}{c|}{ Standard Deviation } \\
\hline \hline Inflow $\left(y_{t}\right)$ & 1 & 1 & 0.242992 & 0.242992 \\
\hline Storage $\left(s_{t}\right)$ & 0.0582519 & 0.088751 & 0.0496936 & 0.059552 \\
\hline Price $\left(P_{t}\right)$ & 1.16418 & 2.25462 & 0.108051 & 0.0381036 \\
\hline $\begin{array}{c}\text { Hydro generation } \\
\left(z_{t}\right)\end{array}$ & 0.986311 & 0.962985 & 0.137723 & 0.0753554 \\
\hline $\begin{array}{c}\text { Gas generation } \\
\left(m_{t}\right)\end{array}$ & 0.747411 & 0.147672 & 0.0759636 & 0.0535763 \\
\hline $\begin{array}{c}\text { Shadow price of } \\
\text { water }\left(W_{s}\right)\end{array}$ & 0.964722 & 0.11452 & 0.0981244 & 0.0690524 \\
\hline $\begin{array}{c}\text { Marginal cost of } \\
\text { gas }\left(M C_{t}\right)\end{array}$ & 1.06312 & 0.210049 & 0.108051 & 0.0762069 \\
\hline \begin{tabular}{c} 
Total surplus $(\mathrm{TS})$ \\
\hline
\end{tabular} & 3.97381 & 3.36082 & 0.126744 & 0.053851 \\
\hline
\end{tabular}


Given the same series of inflows, we observe higher prices and lower quantity in the monopoly market relative to the competitive market. The differences between the prices and quantities in these two markets are considerable, with the average value of the market equilibrium prices in the monopoly market being 86 percent higher than the average value of the market equilibrium prices in the competitive market and the average value of the market equilibrium quantities in the monopoly market being 36 percent lower than the average market quantity in the competitive market, over 30 years.

The reason for having these big differences is that the decision makers in these two markets have different objectives: the monopoly generator aims at maximizing his own profit whereas the social planner aims at maximizing the total surplus to the society which includes not only the surplus to the producers but also the surplus to consumers. As a result, it is typical to see higher quantity and lower prices in a competitive market relative to a monopoly market, as in such a way it transfers some of the producer surplus in the monopoly market into consumer surplus, and captures the otherwise dead-weight-loss (as if in a monopoly market) and in such a way maximizes the total surplus in the society.

The smaller quantity generated in the monopoly market reflects lower levels in both, hydro generation and gas generation. However, we observe that the monopoly generator tends to cut back more on the gas generation than on the hydro generation compared to the social planner's generation decision. As a result, the share of hydro generation in the total generation is significantly different under different market structures - it accounts for only 56.9 percent of total electricity generation if the market is competitive and accounts for 87.5 percent if the market is run by a monopoly generator. This is because if the market is competitive, the social planner chooses to generate a high quantity of electricity in equilibrium, in order to maximize the total surplus to the society. At the equilibrium level, hydro generation itself is not big enough or too costly 
(especially in those low inflow periods) to satisfy all the demand as a result we see on average relatively even weights between hydro and gas generations. However, the monopoly generator chooses the quantity at the level where marginal revenue equals marginal cost. As a result, the total quantity of electricity generated that we observe in the monopoly market is considerably smaller even if they have the same cost structure as the competitive market. However, the supply can be different as the monopoly tends to have lower opportunity cost of .

In equilibrium the monopoly generator generates only a small quantity of electricity in total to some extent relaxes the constraint on the availability of water resources, and therefore decreases the opportunity cost of water. More specifically, given the inflow level, a smaller quantity of hydro generation chosen by the monopoly generator (relative to the amount that would be chosen by the social planner) leads to either a bigger increase in lake level (if the inflow is high), or a smaller decrease in lake level (if the inflow is low), which leads to a lower cost of water in the future period. This decrease in the value of water encourages the monopoly generator to use more hydro in generation as a percentage of its total generation. As a result, comparing to the social planner's choice, the monopoly generator will run the cheap hydro generation, and cut back heavily on the expensive gas generation.

On the other hand, also due to the lower shadow price of water, the monopoly generator turns on hydro generation at lower prices compared to the social planner. Therefore we observe that, although there is a smaller overall hydro generation in the monopoly market relative to the competitive market, the difference between the two hydro generation levels is not as big as for the gas generation. 
Knowing that the monopoly generator in general chooses a lower quantity of generation relative to the social planner, most of the upside fluctuation in the hydro generation and the downside fluctuation in the gas generation will be avoided in the monopoly market. This is because when the water inflow is high, though the water resource is abundant and the marginal cost of water is cheap, the monopoly generator has no interest in generating any more than his unconstrained profit-maximizing level of hydro generation. As a result, in periods when inflows are high, the big increase in hydro generation and decrease in gas generation that we see in the competitive market will not be observed in the monopoly market. This leads to smaller standard deviation of hydro generation, gas generation and price in the monopoly market relative to the competitive market.

Given the same storage capacity and same series of inflows, the monopoly generator tends to put more water in storage than the social planner would choose, and the lake level in the monopoly market is more volatile than it is in the competitive market.

To maximize its profit, the monopoly generator chooses to generate a smaller amount of electricity than the social planner, including hydro generation. The difference between the hydro generation levels in these two markets is particularly significant in periods where the inflows are high. As a result, in periods with high inflows the monopoly generator chooses to use less water in generation and store more in the lake, relative to the social planner. In the dry periods, the social planner would withdraw water from the lake more aggressively to increase the amount of hydro generation (and total generation) in order to restrain the price from becoming too high. On the other hand, the monopoly generator does not have such a problem with having a high price, consequently it would not lower the lake as much as the social planner would in dry periods. As a result the average lake level in the monopoly market over the 
30 years is higher than the average lake level in the competitive market. The difference between the lake levels in the monopoly market and the competitive market is larger when the inflow is high (the storage level chosen by the monopoly in these periods tends to be considerably higher than the level chosen by the social planner, and the monopoly generator tend to fill up the lake more often than the social planner), and lower when the inflow is low. As a result, the monopoly market has a bigger standard deviation of lake level relative to the competitive market.

The difference between the mean of inflow and the mean of hydro generation over 30 years can be considered as a good approximation for the average level of spilling each year ${ }^{21}$. We observe that the monopoly generator on average does much more spilling than the social planner.

The storage lake is more valuable to the social planner than to the monopoly generator. Using the dynamic optimization policies we solved for the monopoly generator in section 5.3.2, we are able to calculate the expected present value of all future profits at the point where the lake is empty and inflow is at its long run mean level (that is $W_{M}(s=0, y=1)$ which equals 57.0064. This value represents the value of an empty lake with a maximum storage capacity of 0.17967 to the monopoly generator ${ }^{22}$. We calculate this value again as if it is in a competitive market. That is, we calculate the expected present value of all future net social surpluses under the competitive policies, which gives $W_{C}(s=0, y=1)=83.9638$. This value gives us the value of an empty lake to the social planner, given that the lake has the same size of 0.17967 . It is 47 percent higher than the value to the monopoly generator.

\footnotetext{
${ }^{21}$ Though in each period the difference between the inflow and the hydro generation may not necessarily be spilling as it may be increasing the lake level, the effect of increasing storage on this difference can be ignored when the average has been take over 30 years of 10950 observations.

${ }^{22}$ It is the value of the storage lake plus all of the other industry structure (i.e., the generators themselves).
} 
The lake is more valuable to the social planner than to the monopolist because it plays a more important role in a competitive market. Instead of serving as just a place to store un-used water rather than spilling it, the storage facility helps the social planner allocate water resource across different periods. This ability to reallocate water resource over time can increase the total quantity of electricity generated in the dry periods, prevent occurrence of very high prices and therefore significantly increases the net social surplus in these periods. As a result, if both the social planner and the monopoly generator can choose the size of storage capacity they want to build, we expect to see that the social planner has an incentive to build a larger storage facility than the monopoly generator.

We measure the benefit to the generator in a given market structure by calculating the profit in that market. We define the profit in a given market in a given date to be the total revenue at the equilibrium market quantity in that period less the generation cost and the transmission cost of gas generation at its equilibrium level and the cost of transmitting the hydro generated electricity. The opportunity cost of water which is captured by the shadow price of water is not included as a cost in this profit calculation. Though its value is taken into account in the generator's decision making, the shadow price of water is an opportunity cost (value in its alternative use in some other future period) rather than an actual monetary cost to the generator has to pay in that period whereas the profit measures the actual monetary return to the generator after paying off all the monetary costs of generation and transmission.

We measure the benefit to society in a given market structure by calculating the net social benefit in that market. Net social benefit in a given market in a given period is defined as the total surplus at the market equilibrium quantity (which is the area under the demand curve up to the market equilibrium quantity) 
taking away the monetary cost of generation and transmission which include the generation cost and transmission cost of gas-generated electricity at the equilibrium level, and the cost of transmitting the hydro-generated electricity. Similarly to the profit calculation, the opportunity cost of water is also excluded from the net social benefit calculation as it is not an actual monetary cost.

The profit and the net social benefit in the competitive market and in the monopoly market at their own equilibrium quantities is calculated in each period over 30 years. The mean of the profit and the net social benefit in these two markets are summarized in the following table (Table 5-3):

\begin{tabular}{|c|c|c|}
\hline & Competitive market & Monopoly market \\
\hline Profit & 1.39434 & 2.27974 \\
\hline Net social surplus & 3.9384 & 3.35251 \\
\hline
\end{tabular}

Table 5-3: Mean of profit and net social surplus in the two markets

The average level of profit per day in the monopoly market is 63.5 percent higher than the average level of daily profit earned in the competitive market. That is, the under-generation and over-pricing exercised by the monopoly generator relative to the social planner's social surplus maximizing price and quantity choice have earned the monopoly generator a big profit.

Besides the fact that the monopoly generator maintains a much higher profit than the social planner, each market's profit response to inflow shocks is also different. The profit level in the competitive market tends to fluctuate a little over time and profit decreases significantly when high inflow comes while the lake is full. This is because in the competitive market electricity is supplied at its marginal cost, the profit in this market arise from the opportunity cost of water as it is a cost that has been taken into consideration for deciding the optimal generation but is not deducted from the revenue in profit calculation as it does 
not associate with any cash out-flow. In the periods where the storage is close to being full or is full and the inflow is high, the stored water loses a considerable amount of value. Additionally, in these periods since the storage is full the inflow has to be used in generation or be spilled (if the hydro plant reaches its maximum generation capacity). The social planner will choose to use hydro generation as much as he can which leads to a big increase in the total quantity generated and a big decrease in the market price. Particularly low price and high quantity bring the social planner a much lower profit. Therefore we observe a significant decrease in profit in the competitive market during these periods.

On the other hand, the monopoly generator maintains the profit at a high level steadily without much fluctuation. This is because in these periods, the monopoly generator only runs the hydro generation plant up to the unconstrained profit-maximizing quantity. The remaining inflow in excess of the needed amount will be spilled. As a result the price in the monopoly market is well maintained above a certain level and therefore no significant decrease in profit occurs during these periods. However, we do observe decreases in the profit level when low inflow occurs while the lake is close to being empty. This is because in these periods, the amount of water resource is limited and there is not much the monopoly generator can do about it because the lake level is also low (close to or equal to zero). As a result, the monopoly generator has to decrease the hydro generation and substitute some with the expensive gas generation, which leads to high price and low quantity in these periods and brings the monopoly generator a lower profit.

The social planner does bring a higher net social benefit to the society than the monopoly generator in each period. The average level of net social benefit per day in the competitive market is 17.5 percent higher than the average level of daily net social benefit produced in the monopoly market. The lower price and 
higher quantity of generation chosen by the social planner could make the society much better off relative to the monopoly generator.

In the competitive market, the net social benefit increases significantly in periods when hydro generation is particularly high. This occurs in periods with high inflows while the storage lake is full. In these periods, the non-availability of the storage lake and the abundance of the water resource from the inflow give the social planner incentive to generate a small amount of electricity using gas at low cost and a large amount of hydro-generated electricity subject to the generation capacity of the hydro plant. The large quantity of electricity is supplied to the market at a low price. The low price and high quantity bring a big total surplus. After taking away the low cost of gas generation (because only a small quantity is generated) and the transmission costs, the society is left with a big net social benefit in these periods.

On the other hand, social benefit decreases in periods when the inflow is low while the storage is empty. In these periods, the social planner has very little water that can be used in hydro generation. This situation can not be improved by the existence of the lake as there is no water in the lake. As a result, the social planner has to substitute hydro generation with the more expensive gas generation. Overall in equilibrium low quantity will be supplied to the market at high price which leads to a low net social benefit to the society.

Though the net social benefit gained in the monopoly market is much lower in the competitive market, similar decreases in the net social benefit during these low inflow empty storage periods are also found in the monopoly market. However, no high net social benefit is observed in the monopoly market in periods when the inflow is high and the storage is full because unlike the social planner the monopoly generator does not use all the inflow in hydro generation. To maximize its profit, the monopolist only runs the hydro plant up to the un- 
constrained profit maximizing level. Therefore the price in the monopoly market is maintained in these periods and society does not get the much benefit of a low price or high quantity as it does from the competitive market.

The difference between the net social benefit gained in the competitive market and the net social benefit gained in the monopoly market in each period measures the loss of social welfare by having absolute market power in the market. The loss caused by market power is greater in periods where the monopoly generator is able to withhold back the generation when there are enough resources for a large quantity to be generated at low cost (i.e. when the inflow is high and the lake is full). The loss to the society is smaller in periods when the inflow is small and the lake is empty, because in these periods both the social planner and the monopoly generator are constrained by the availability of water and both markets will have a relatively low net social benefit. However, the social planner always chooses a higher quantity of total generation than the monopoly generator and in these dry periods most of this difference in generation levels is generated by the gas plant. Therefore, although the social planner in equilibrium supplies a higher quantity at lower price relative to the monopoly generator which brings a higher total surplus to the market, the additional cost of generating more gas-generated electricity at increasingly high cost, and additional transmission costs make the loss to the society caused by market power smaller in dry periods. 
Diagram 5-22: Comparison between the market outcomes generated in a competitive market and in a monopoly market (blue curve for the competitive case and purple curve for the monopoly case):

Storage level

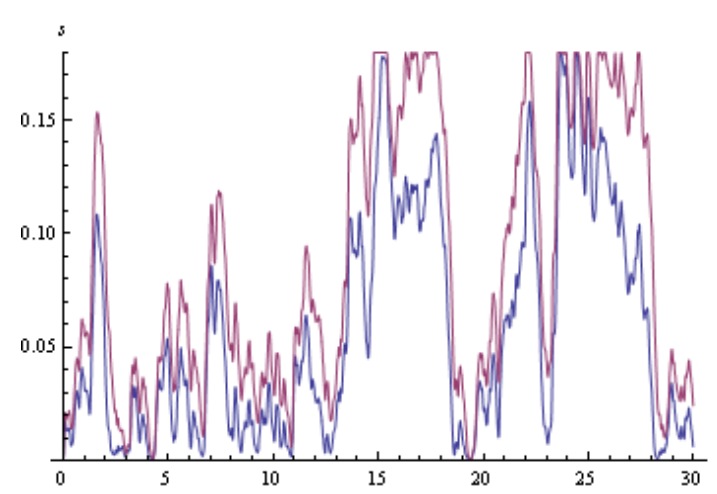

Hydro generation

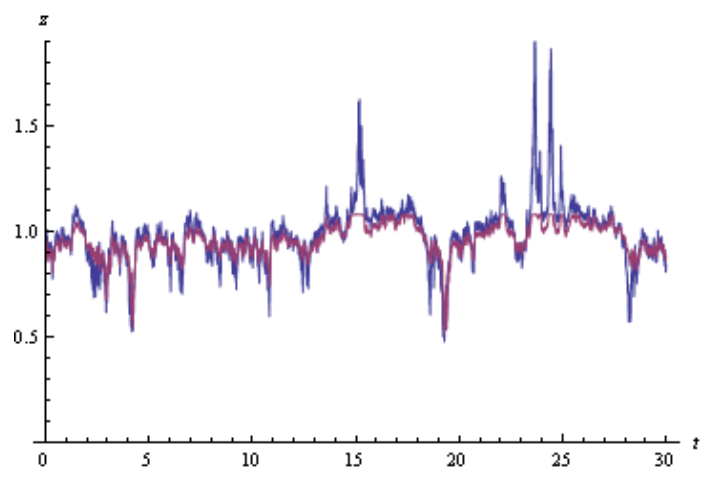

Equilibrium price

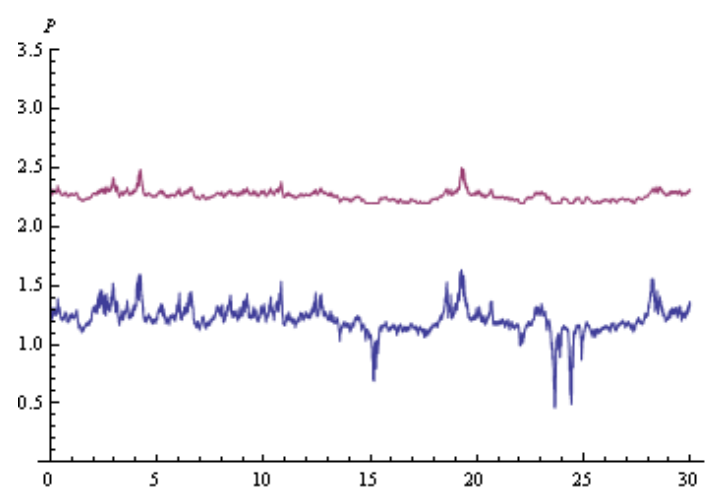

Shadow price of water

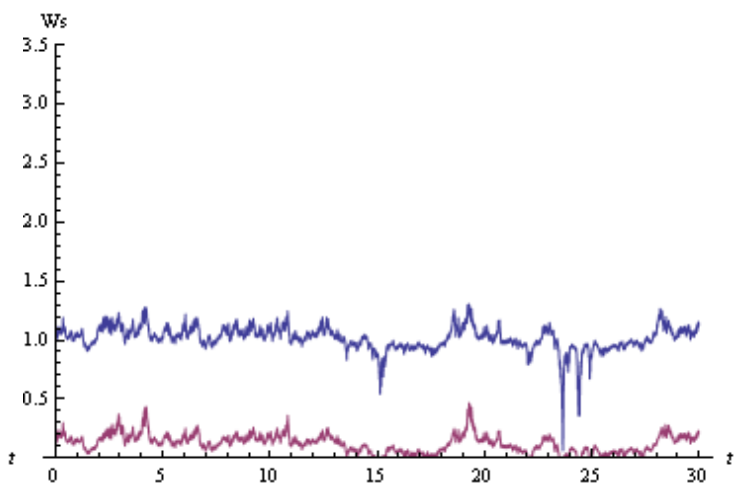

Gas generation

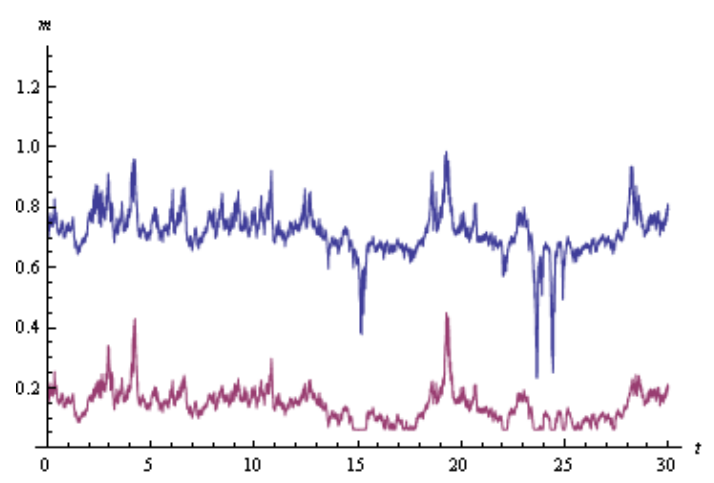

Net Social benefit

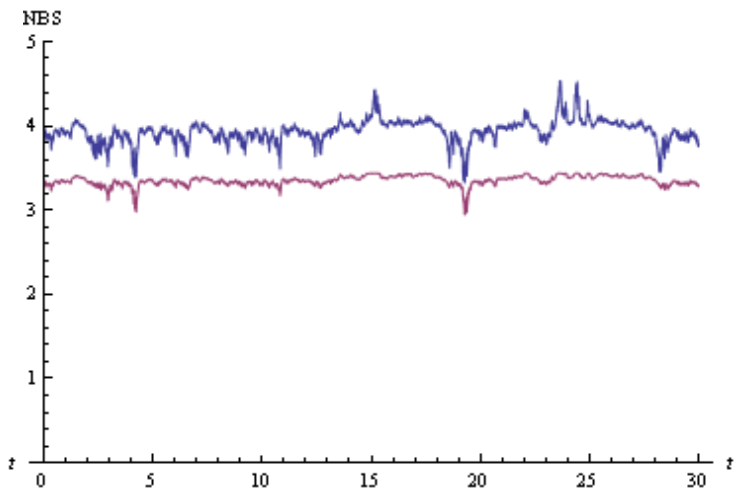




\section{Section 6 Comparative statics analysis}

\subsection{Overview}

In the previous section, we presented the equilibrium market outcomes of the standard New Zealand calibrated model given the simulated 30 years of daily water inflow, assuming the perfect competitive market. In this section, we will undertake a number of comparative statics of the standard model by altering some market condition. We consider the effect of different prices for gas. We also explore variation in climatic related variable which is the river inflows.

\subsection{Comparative statics of the competitive model}

\subsubsection{Capacity of the gas generation plant}

In this section we analyze the impact that the generation capacity of the gas generation plant has on the market outcomes in the competitive setting, in particular when the capacity of low marginal cost gas generation changes. We do this by changing the maximum capacity of the gas generation, rotating the marginal cost function of the gas generation so that the marginal cost of generating the maximum amount of gas generation is still the same, equal to the market clearing price minus the transmission cost. In this way by increasing or decreasing the generation capacity we are actually adding or taking away the low marginal cost portion of gas production. Having a small generation capacity would imply a higher marginal cost at each given quantity.

In the standard model we set the maximum generation capacity of the gas generation equal to 1.33454 . We will compare the market outcomes generated under the standard setting with the ones generated given a $60 \%$ increase and 
decrease in the maximum generation capacity of gas generation to study the impact of the gas generation capacity on the market outcomes in a competitive market. Table 6-1 summarizes the market outcomes given these three different gas generation capacity.

Table 6-1: market outcomes at different generation capacity of the gas plant

\begin{tabular}{|c|c|c|c|c|}
\hline Quantity & \multicolumn{2}{|c|}{$60 \%$ higher $\bar{m}$} & \multicolumn{2}{c|}{$60 \%$ lower $\bar{m}$} \\
\hline Inflow $\left(y_{t}\right)$ & 1 & 0.24299 & 1 & $\begin{array}{c}\text { Standard } \\
\text { Deviation }\end{array}$ \\
\hline \hline Storage $\left(s_{t}\right)$ & 1.027523 & 1.180555 & 0.988736 & 1.002194 \\
\hline Price $\left(P_{t}\right)$ & 0.778922 & 0.759281 & 1.427616 & 1.479907 \\
\hline $\begin{array}{c}\text { Hydro generation } \\
\left(z_{t}\right)\end{array}$ & 0.999901 & 1.000603 & 1.00004 & 1.00016 \\
\hline $\begin{array}{c}\text { Gas generation } \\
\left(m_{t}\right)\end{array}$ & 1.21421 & 1.214855 & 0.585885 & 0.575156 \\
\hline $\begin{array}{c}\text { Shadow price of } \\
\text { water }\left(W_{s}\right)\end{array}$ & 0.73714 & 0.769888 & 1.509068 & 1.461834 \\
\hline $\begin{array}{c}\text { Net social benefit } \\
(\mathrm{NSB})\end{array}$ & 1.046712 & 0.732808 & 0.909735 & 1.513156 \\
\hline
\end{tabular}


Diagram 6-1: Comparison between the market outcomes generated in a competitive market given three different generation capacities of the gas generation (purple for the case with dam size in the standard calibration, yellow for the case with $60 \%$ larger generation capacity of gas and blue for the case $60 \%$ lower generation capacity of gas):

Storage

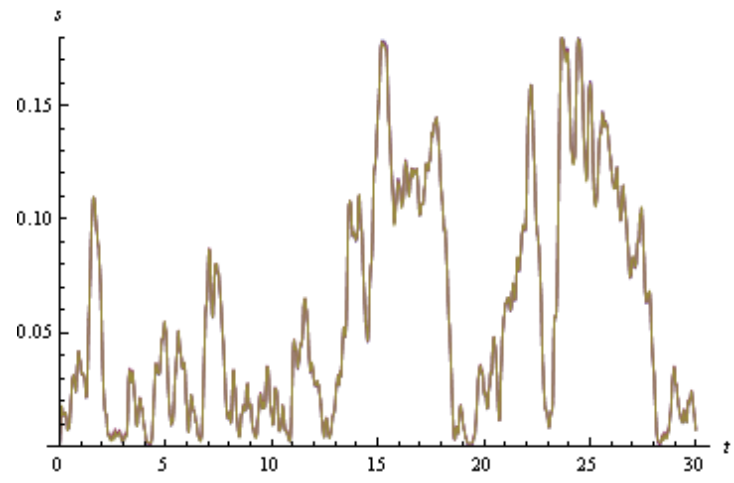

Hydro

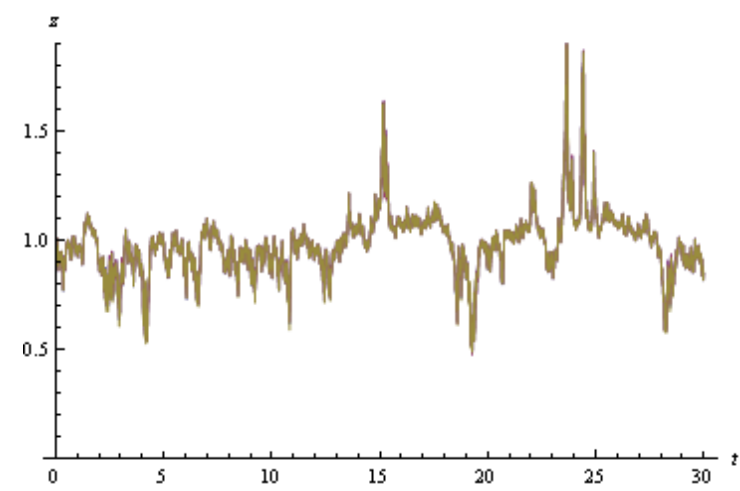

Price

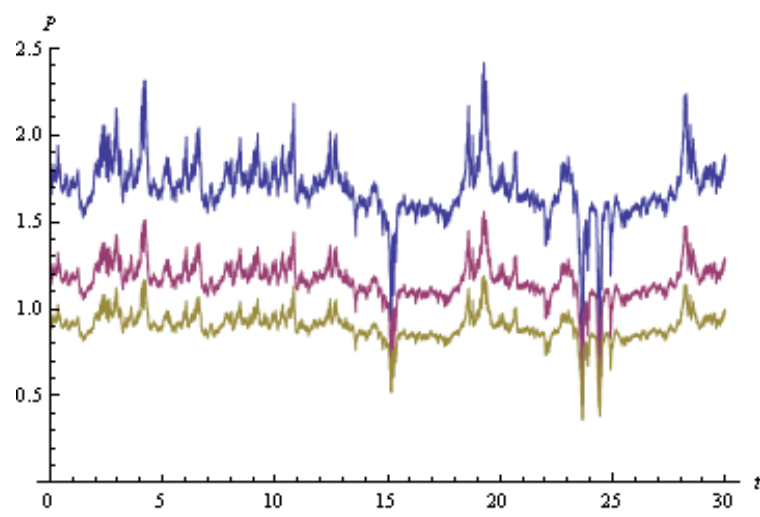

Shadow price of water

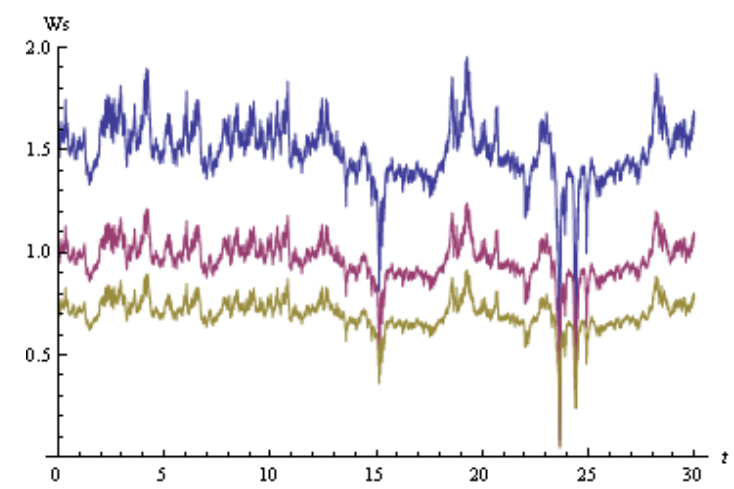

Gas
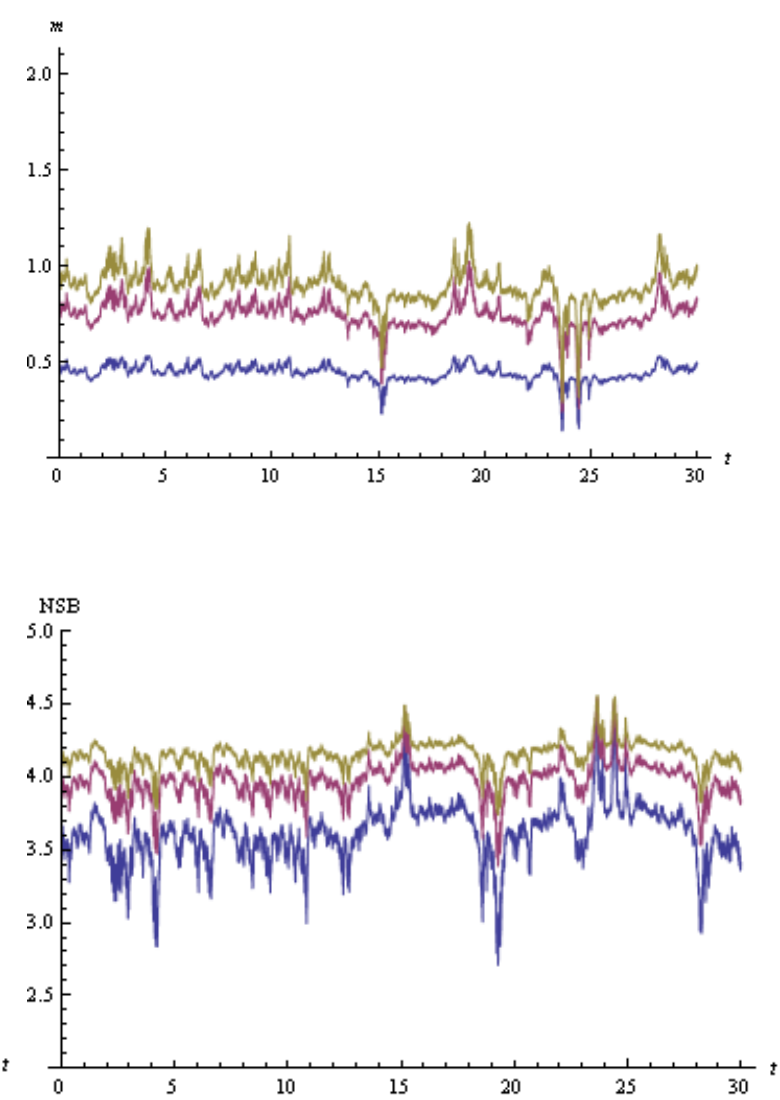

We find that given an increase in the gas generation capacity there is a significant increase in the amount of gas generation used at equilibrium. It is obvious that an increase in the low marginal cost gas generation capacity leads 
to an increase in the amount of gas generation at equilibrium in a competitive market. This is because an increase in the low marginal cost capacity lead to a lower marginal cost for any given quantity which encourages the social planner to use more gas generation.

Also, when the gas generation capacity is low the generation capacity constraint might actually become binding in certain periods when there is not enough water available. For example, in our small capacity case where we cut $60 \%$ off the gas generation capacity from the standard calibration, we observe that in some periods for example towards the end of the $4^{\text {th }}$ year and in the mid of year 19 , the gas generation reaches its maximum capacity. Increasing gas generation capacity from low to medium does not only reduce the marginal cost for each given quantity but also allows more generation in periods with low hydro resources by relaxing the capacity constraint. That is why we observe a bigger increase in the gas generation level when we move from the small capacity to the standard capacity. By moving from the standard capacity to the big capacity while we still observe an increase in the gas generation but the difference is not as big as if we are moving from a small capacity to a standard one. This is because in both the standard case and the big capacity case the capacity is big enough for the social planner to choose the generation quantity without being constrained: the increase in average gas generation is a result of the cheaper marginal cost, not from relaxing the capacity constraint. 


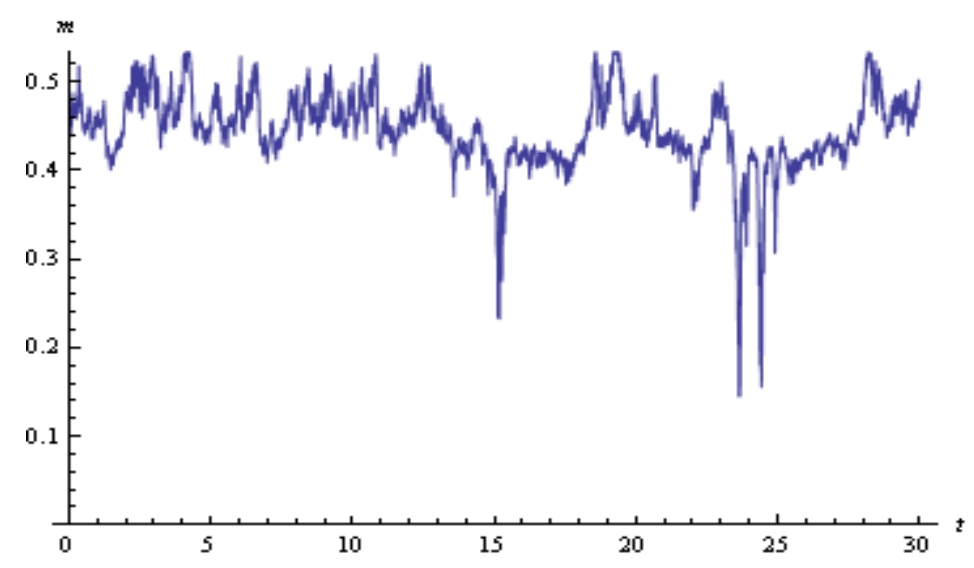

Diagram 6-2: Gas generation given the low capacity of the gas plant
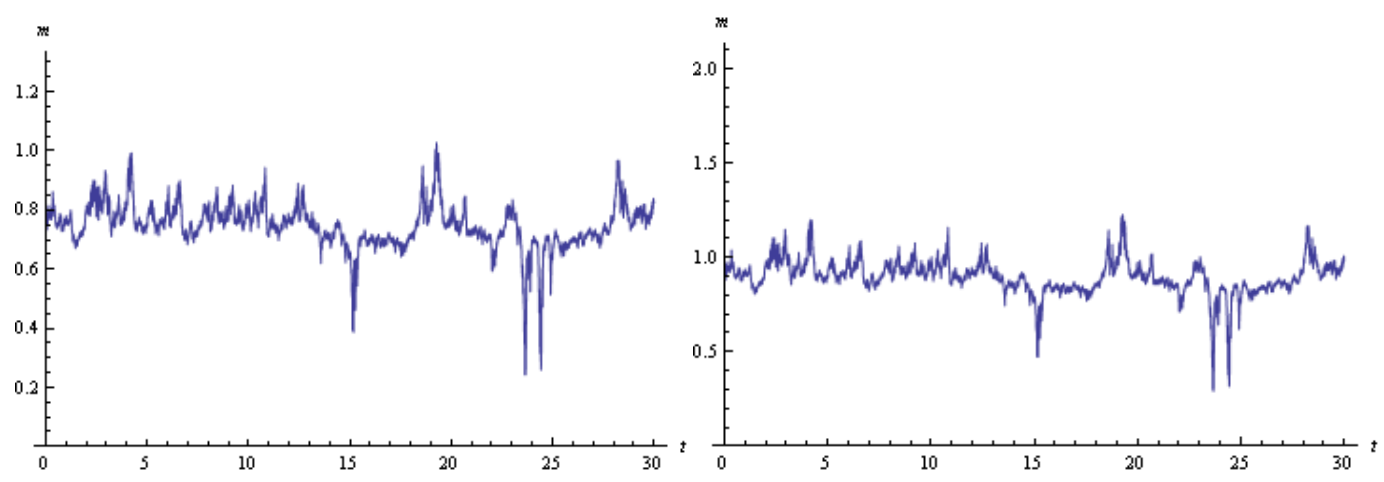

Diagram 6-3: The gas generation level given for the standard gas generation capacity (left) and the big gas generation capacity (right).

While the gas generation level increases significantly with increases in the gas generation capacity, we do not observe a significant change in the hydro generation level and in the lake level.

Although the storage level and the hydro generation level remain unchanged, the shadow price of water changes quite significantly as the gas generation capacity changes. We observe that the shadow price of water is higher when the gas generation capacity is small and the shadow price is lower when the gas generation capacity is big. One way to understand this result is that we can think of having a small gas generation capacity as an outage of low marginal cost gas 
plant or a positive shift in demand - when there is an outage in the supply of the gas generated electricity or when there is a positive shift in demand the hydro resource becomes more important which leads to an increase in the shadow price of water. The other way to think about it is that the shadow price of water which measures the value of the hydro resource also reflects the marginal cost of its substitute. As we reduce the maximum level of gas generation capacity we also make the marginal cost for any given quantity becomes more expensive. Therefore up-scaling in the marginal cost of gas generation lead to an up-scale in the shadow price of water.

As a result of the changes in the gas generation level, the marginal cost of gas generation and the shadow price of water given the variation in the gas generation capacity, we observe some significant differences in the competitive equilibrium prices under the three cases. Similar to the shadow price of water, the price to consumer is higher when the gas generation capacity is small and decreases as we increase the capacity.

Given the results we have seen so far, an outage in the low marginal cost gas generation plant generally leads to a lower quantity of electricity generated (which is mainly due to the decrease in the amount of gas generation) and an increase in the competitive per unit price of electricity in the market. These two effects collectively lead to a decrease in the net social benefit in society. We found that the net social benefit increases as the gas generation capacity increases, but at a decreasing rate. Outage in the gas generation capacity or positive demand shock in general lead to decrease in welfare and this effect is particularly significant when the generation capacity is already low. 


\subsubsection{Water inflow process}

In our model we assumed that nature generates water inflow $y_{t}$ at each time $\mathrm{t}$ according to the following square-root process:

$$
d y_{t}=\eta\left(\mu-y_{t}\right) d_{t}+\sigma \sqrt{y_{t}} d \xi_{t}
$$

With $\eta$ measuring the rate of mean reversion, $\mu$ measures the long term mean and $\sigma$ measures the volatility of the inflow process.

After fitting the square root model to monthly water inflow data from a centralised dataset issued by the New Zealand Electricity Commission, we obtained estimates for the parameters of the inflow process:

$$
d y_{t}=6.9448\left(1-y_{t}\right) d_{t}+0.9056 \sqrt{y_{t}} d \xi_{t}
$$

As a result, for the standard competitive model we set the rate of mean reversion $\eta$ equal to 6.9448 , the long term mean $\mu$ equals 1 and the volatility measure $\sigma$ equals 0.9056 .

In this section we conduct comparative analysis to study how the market outcomes change as a result of changes in two inputs for the water inflow process - the mean and the rate of mean reversion. For each of the two parameters we will generate the outcomes in the competitive market using the value of the parameter plus and minus $30 \%$ of its value used in the standard case, while keeping the other two parameters equal to the original levels. By comparing these newly generated results with the ones we obtained in the standard model, we hope to understand the impact these inputs have on the market outcomes in the competitive market. 


\subsection{2 - 1 Mean of water inflow process $(\mu)$}

First, we compare the market outcomes generated in the markets with different long term means of the water inflow process, more specifically when $\mu$ equals 0.7, 1 and 1.3.

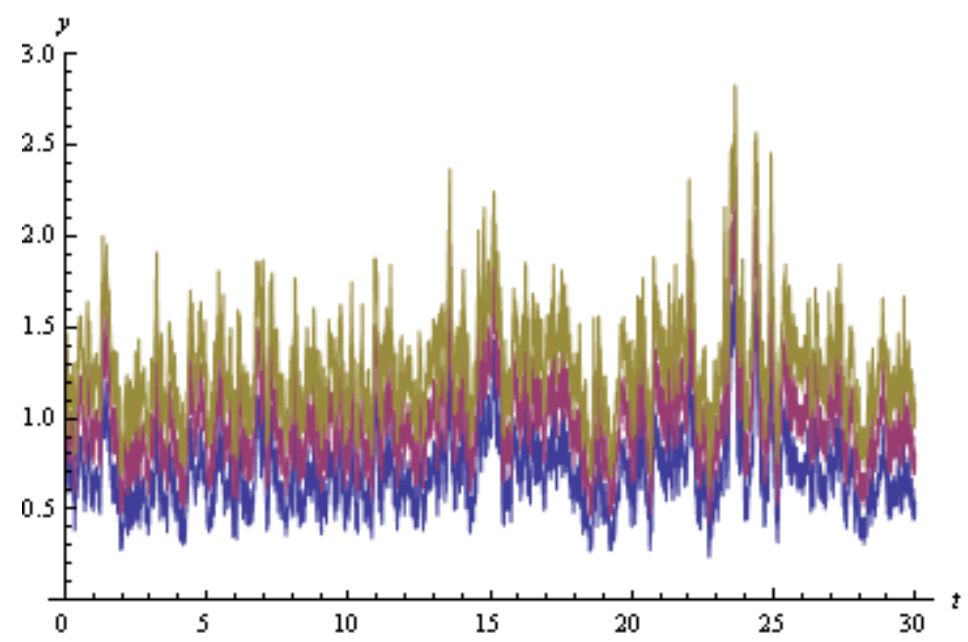

Diagram 6-4: Simulated water inflow given different values of the mean

Diagram 6-4 demonstrates what the simulated inflow processes look like with different values of $\mu$. The blue line represents the simulated daily inflow with $\mu$ equal to 0.7 ; the purple line represents the simulated inflow given that $\mu$ equals 1 (which is the value used in the standard model); and the yellow line represents the simulated inflow given that $\mu$ equals 1.3. This diagram shows that changes in the long term mean $\mu$ while keeping the rate of mean-reversion and volatility measures constant, do not change the movement in the inflow process, instead it shifts the entire inflow process up or down. In order to compare results from different inflow series, we retain the simulated draws. That is the higher the $\mu$, the more inflow there is in each given period.

The following table summarizes the market outcomes in a competitive market given three different values for the long term mean $\mu$. 


\begin{tabular}{|c|c|c|c|c|}
\hline \multicolumn{2}{|c|}{ Table 6-2: market outcomes at different average inflows ${ }^{23}$} \\
\hline Quantity & \multicolumn{2}{|c|}{$30 \%$ higher $\mu$} & \multicolumn{2}{|c|}{$30 \%$ lower $\mu$} \\
\hline Mean & $\begin{array}{c}\text { Standard } \\
\text { Deviation }\end{array}$ & Mean & $\begin{array}{c}\text { Standard } \\
\text { Deviation }\end{array}$ \\
\hline Inflow $\left(y_{t}\right)$ & 1.3 & 0.277053 & 0.7 & 0.203301 \\
\hline Storage $\left(s_{t}\right)$ & 1.0777004 & 1.0360469 & 0.850906151 & 0.926704847 \\
\hline \begin{tabular}{c} 
Price $\left(P_{t}\right)$ \\
\hline $\begin{array}{c}\text { Hydro generation } \\
\left(z_{t}\right)\end{array}$
\end{tabular} & 0.80048876 & 1.09634339 & 1.200664846 & 0.81685778 \\
\hline $\begin{array}{c}\text { Gas generation } \\
\left(m_{t}\right)\end{array}$ & 0.78152583 & 1.09634483 & 1.219745227 & 0.816858337 \\
\hline $\begin{array}{c}\text { Shadow price of } \\
\text { water }\left(W_{s}\right)\end{array}$ & 0.0533676 & 1.17400973 & 1.24134206 & 0.800082344 \\
\hline $\begin{array}{c}\text { Net social benefit } \\
(\mathrm{NSB})\end{array}$ & 1.0630729 & 0.8572319 & 0.91934944 & 1.044112542 \\
\hline
\end{tabular}

We observe that as the long term mean of the inflow $\mu$ increases, the lake level increases. This is because higher $\mu$ implies more inflow in all periods. With more water in each given period the social planner will choose to increase the amount of water to be stored accordingly. However, the magnitude of changes in the lake level is small in periods when the inflows are low or when the lake is already full and bigger in periods when the inflows are high. This is because given an increase in the hydro inflow mean, the changes in the inflow level given an increase in the mean are positively correlated with the fluctuations in the inflow itself. That is, the increase in inflow is bigger when the inflow is high and smaller when the inflow is small, which causes the storage level to increase significantly during these wet periods relative to the drought periods. We also observe that for those periods where the lake is full in the standard model where $\mu$ equals 1 , decreasing $\mu$ leads to a decrease in the lake level while increasing

\footnotetext{
${ }^{23}$ Data except inflows are relative to the standard calibration.
} 
$\mu$ does not increase the storage level any further due to the fact that the lake has already reached its maximum capacity.

An increase in the mean of the inflow process $\mu$ increases the amount of water available to the social planner in each given period, in both the direct way though the higher inflow in each period and the indirect way though the higher amount of water in the lake stored in the past. As the water becomes less scarce, an increase in $\mu$ leads to a decrease in the shadow price of water in that market. This decrease lowers the opportunity cost of water which encourages the social planner to use more hydro in electricity generation. That is why we observe a significant increase in the amount of hydro generation chosen by the social planner given the increases in $\mu$. On the other hand, the gas generation becomes relatively more expensive to hydro generation as the mean of inflow increases. Therefore we observe decreases in the amount of gas generation as the inflow mean increases.

Overall increases in the mean of the inflow significantly increase the total quantity of electricity at equilibrium and significantly decrease the overall price in the market. Moreover it brings a higher welfare to the society as it allows the social planner to use more hydro in generation in each period at lower cost and with more water in the storage. However, it is worthwhile to note that the increase in the net social benefit is less significant in periods when the lake is close to being empty or full - that is when the lake's ability to smooth the hydro generation is limited. 
Diagram 6-5: Comparison between the market outcomes generated in a competitive market given three inflows with different mean (Blue for the case with low mean, purple for the case with the standard mean and yellow for the case with high mean):

Storage

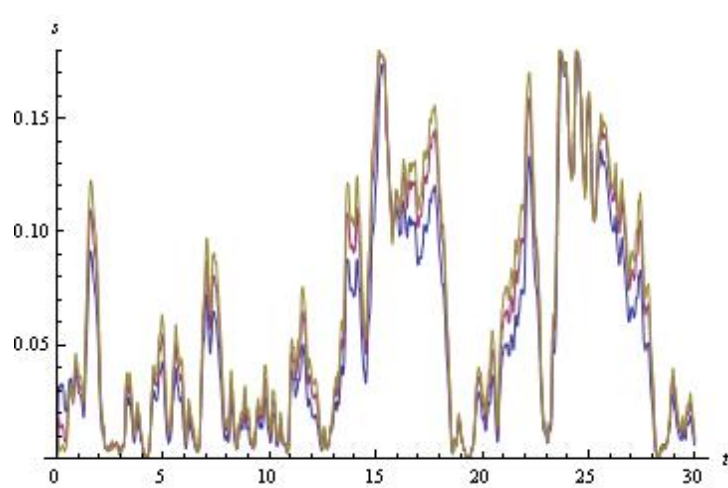

Hydro generation

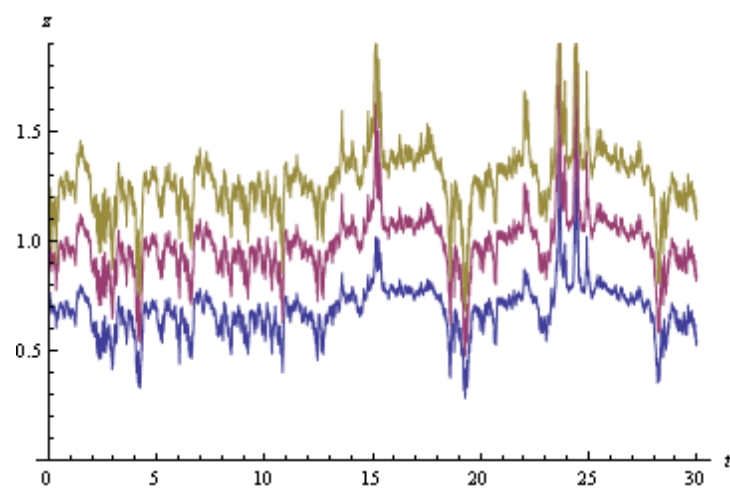

Price

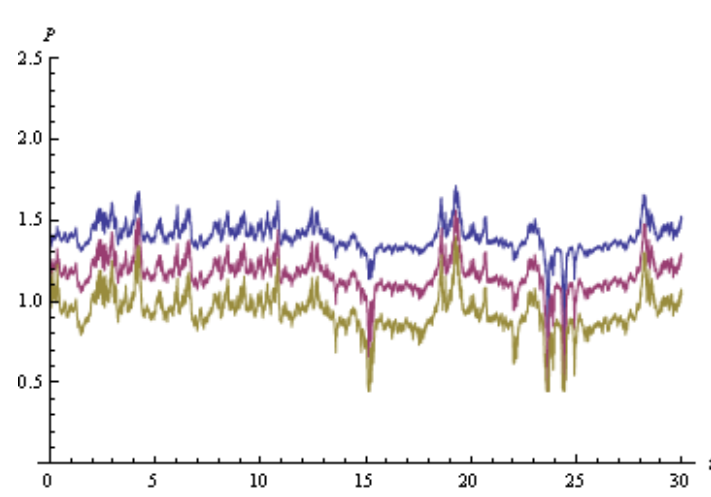

Shadow price of water

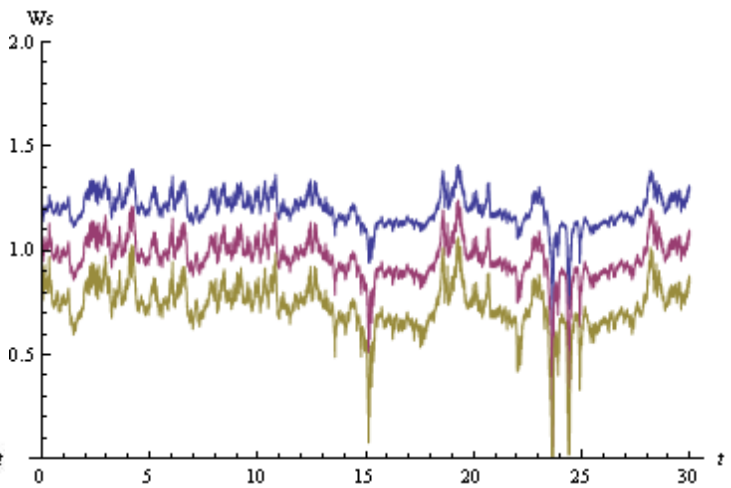

Gas generation

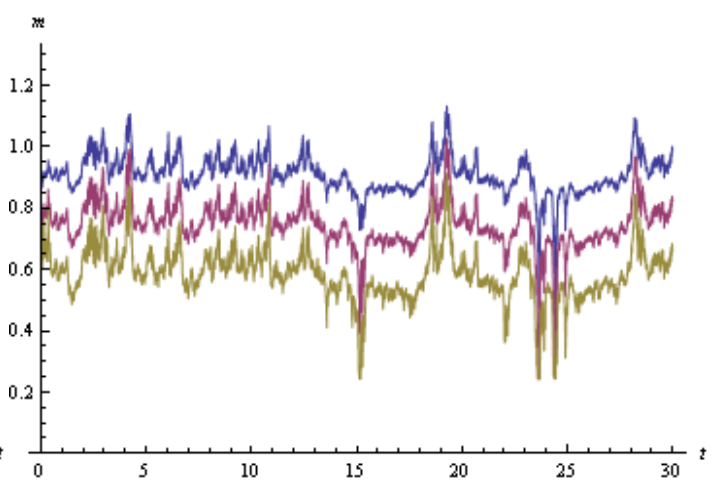

Net social benefit

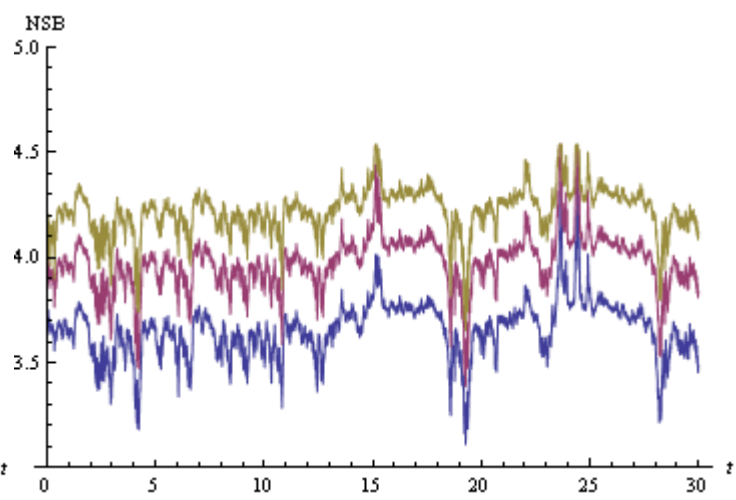




\subsection{2 - 2 Mean reversion rate of water inflow process $(\eta)$}

Now we compare the market outcomes generated in the competitive market given three different simulated water inflow using different values for the rate of mean-reversion, more specifically for values of $\eta$ equal to the value estimated using the New Zealand inflow data, and when it equals plus and minus $30 \%$ of the standard value.

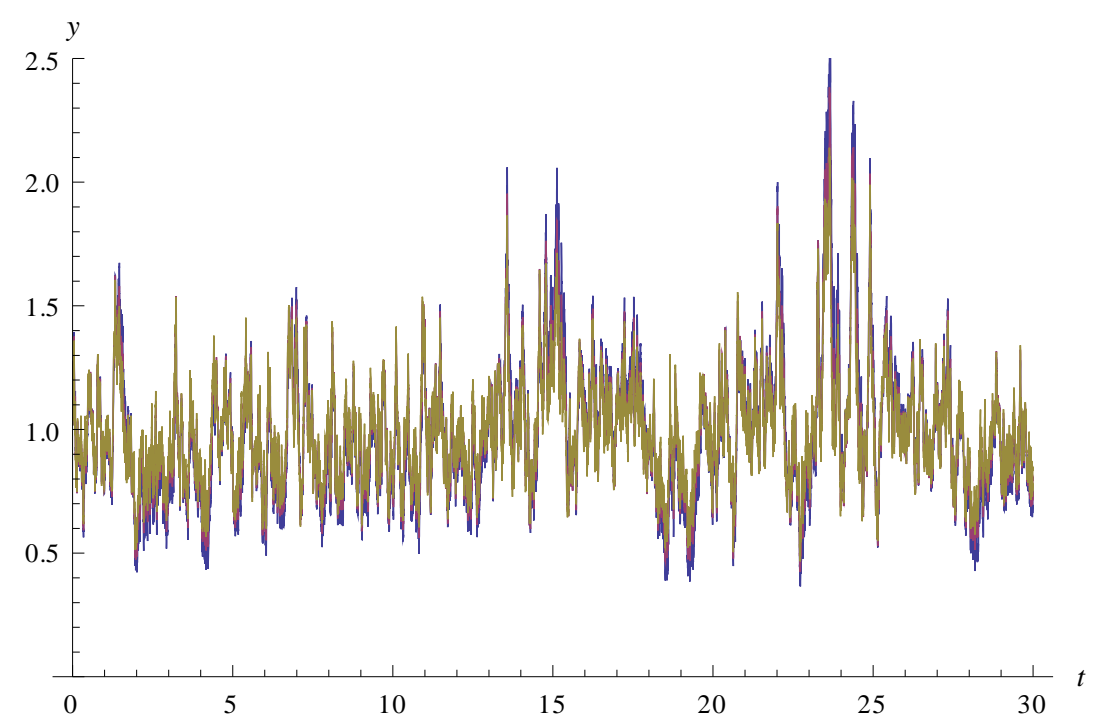

Diagram 6-6: Simulated water inflow given different values of the mean reversion rate.

The mean reversion rate measures the rate by which the shocks dissipate and the variable reverts towards the mean. By comparing the three simulated inflow using different values of the mean reversion rate in diagram 6-6, we can see for any given random shock that diverts the inflow away from its mean it takes less time for the simulated inflow with high $\eta$ to adjust back toward its mean, relative to the one simulated using lower values for $\eta$. As a result of the inflows simulated using the higher mean reversion rate are more stable over time and the fluctuations in the process are smaller, so that the inflow is closer to the mean. 
We then run the competitive model on each of the three simulated series of inflow and generated the market outcomes at equilibrium under each scenario. Table 6-6 below summarizes the results.

\begin{tabular}{|c|c|c|c|c|}
\hline \multicolumn{2}{|c|}{ Table 6-3: market outcomes at different inflow mean reversion ${ }^{24}$} \\
\hline Quantity & $30 \%$ higher $\eta$ & \multicolumn{2}{|c|}{$30 \%$ lower $\eta$} \\
\hline Mean & $\begin{array}{c}\text { Standard } \\
\text { Deviation }\end{array}$ & Mean & $\begin{array}{c}\text { Standard } \\
\text { Deviation }\end{array}$ \\
\hline Inflow $\left(y_{t}\right)$ & 1 & 0.213 & 1 & 0.290 \\
\hline $\begin{array}{c}\text { Storage }\left(s_{t}\right) \\
\text { Price }\left(P_{t}\right)\end{array}$ & 0.95999959 & 0.9287856 & 1.024651556 & 1.098332582 \\
\hline $\begin{array}{c}\text { Hydro generation } \\
\left(z_{t}\right)\end{array}$ & 1.0033823 & 0.75653304 & 0.993445272 & 1.388126892 \\
\hline $\begin{array}{c}\text { Gas generation } \\
\left(m_{t}\right)\end{array}$ & 0.99753817 & 1 & 1.004771137 & 1.388125365 \\
\hline $\begin{array}{c}\text { Shadow price of } \\
\text { water }\left(W_{s}\right)\end{array}$ & 0.99811552 & 0.74554341 & 1.003206105 & 1.423641826 \\
\hline $\begin{array}{c}\text { Net social benefit } \\
(\mathrm{NSB})\end{array}$ & 1.00161306 & 0.77259357 & 0.9966858 & 1.372609354 \\
\hline
\end{tabular}

Having a higher mean reversion rate smoothes the fluctuations in the inflow process over time and it reduces the unconditional variance of inflow. Therefore it is not surprising to see that the impact of a higher mean reversion rate is very similar to that we have seen just before when we used a lower standard deviation measure of the inflow process. On average the market with inflow that has a higher mean reversion has a lower lake level in each period and a lower shadow price of water; the lower shadow price of water encourages the social planner to use more hydro in each period on average and decreases the amount of gas generation which overall leads to a lower price in the market and results a higher welfare for society.

\footnotetext{
${ }^{24}$ Data except inflows are relative to the standard calibration.
} 
Diagram 6-7: Comparison between the market outcomes generated in a competitive market given three inflows with different mean reversion rates (Yellow for the case with low mean reversion rate, purple for the case with the mean reversion rate used in the standard case and blue for the case with high mean reversion rate):

Storage

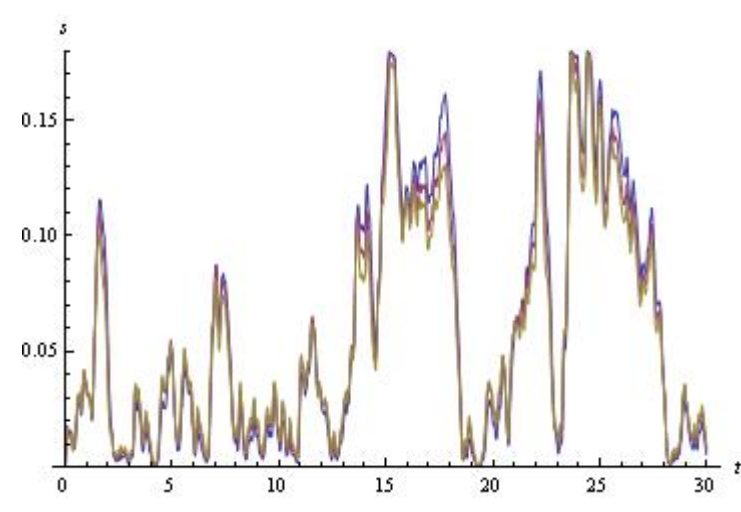

Shadow price of water

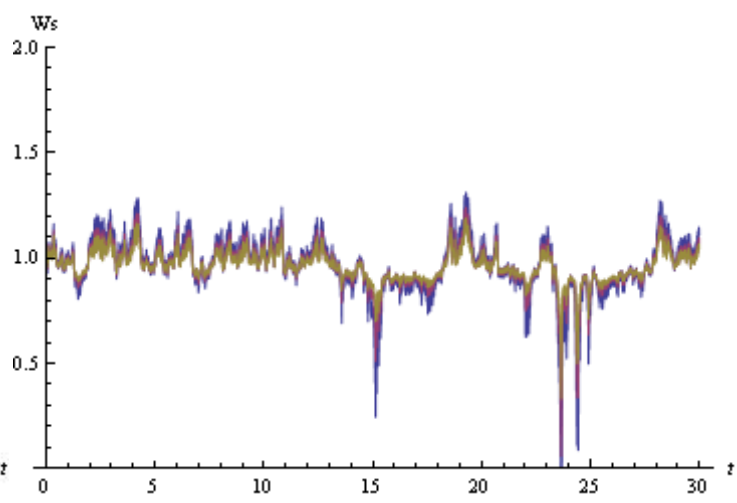

Hydro generation

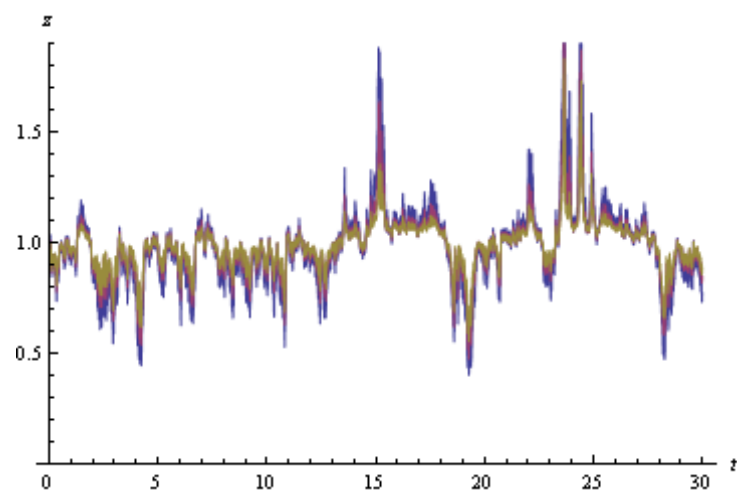

Gas generation

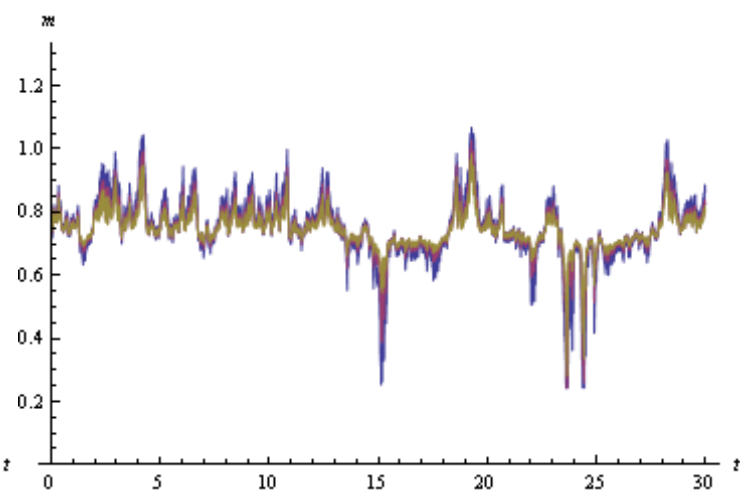

Price

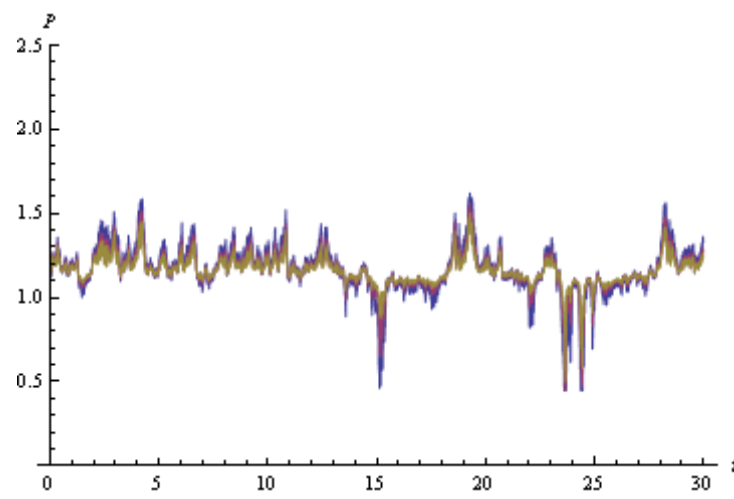

Net social benefit

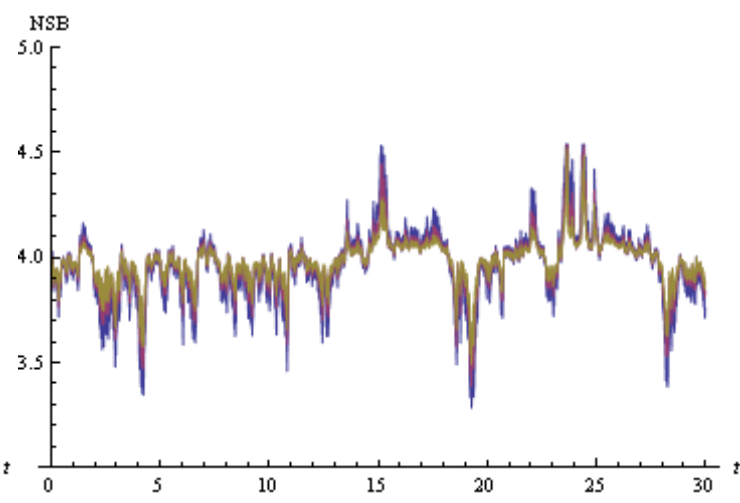




\section{Section 7: Methods for assessing the exercise of market power in electricity markets}

In this section, we analyze and evaluate the methods for assessing the exercise of market power in electricity markets. Section 7.1 presents a literature review on the methods that have been used to assess the exercise of market power in the past. In section 7.2 we will discuss the approaches that can be used to assess the existence of market power abuse in the New Zealand market. Finally in section 7.3 we will use the market outcomes generated in our simulation to evaluate the effectiveness of the market power test in the New Zealand market.

\subsection{Literature review on the methods for assessing the exercise of market power in electricity markets}

There are many factors that affect the level of market power abuse in a particular market, these includes: number of participants, relative size of each supplier, elasticity of demand where there are many, and no dominant, demanders, barriers to entry, the amount and distribution of excess capacity, transaction costs of trade and factors such as strategic and environmental uncertainty. ${ }^{25}$

Studies of electricity markets have generally assumed that there are many pricetaking demanders, and that strategic and environmental uncertainties can be neglected. ${ }^{26}$

\footnotetext{
${ }^{25}$ Strategic uncertainty arises when there is uncertainty about the behaviour of other market participants. Environmental uncertainty is the uncertainty that is due to nature: for example in electricity markets this would include uncertainty about the gas price and lake inflows.

${ }^{26}$ See Evans and Guthrie (2009) for analysis of the effect of fuel availability and price on electricity production decisions.
} 


\subsubsection{Concentration measure}

Abuse of market power is a problem inherently related to the structure of a market. In a monopoly market, the monopolist has absolute market power because he is the only supplier in the market and therefore determines the market price and quantity. However, in a market where there is a large number of small firms, each firm's behaviour will have only small, if any, effects on the market equilibrium price and quantity. Therefore the potential of market power abuse in a particular market is positively correlated to the concentration in that market.

The Herfindahl-Hirschman Index is the most widely used index for structural assessment of a market. It is defined as the sum of the squares of the market shares of the 50 largest firms (or summed over all the firms if there are fewer than 50 firms) in the industry. The market share is measured in percentage value.

$$
H H I=\sum_{i=1}^{N} s_{i}^{2}
$$

The HHI approaches 0 when there are a large amount of small suppliers in the market (perfectly competitive) and it equals 10000 when the market is pure monopoly.

The Herfindahl-Hirschman Index is easy to calculate and it provides information about the concentration in this market. Additionally, $\mathrm{HHI}$ takes account of the relative size of all suppliers in the market, and proportionally gives greater weights to bigger firms.

This HHI is adopted by some US regulatory agencies such as the Federal Energy Regulation Commission (FERC) and the Department of Justice (DOJ) in United 
States as a screening test in determining whether an electricity supplier is likely to have market power. This is because all else equal the potential for market power in a market is correlated with its market concentration. When the market is heavily concentrated, a lack of competition will make it easier for the firms in such a market to exercise market power. Markets with HHI values between 1000 and 1800 are considered to be moderately concentrated, and those with HHI exceeding 1800 are considered to be concentrated. Under the horizontal Merger Guideline issued by DOJ in United States, in the case when a single transaction leads to a 100 points increase in HHI in a concentrated market, an antitrust concern is raised.

However, this approach is generally not used as a direct measure of market power in electricity markets. This is because the index does not take into account other factors that play important roles in determining the level of market power abuse, such as the elasticity of demand, the competitive cost of generation, barriers of entry, capacity constraints etc. Borenstein (1999) showed that the HHI does a poor job of measuring the amount of market power in electricity markets, due to the fact that the electricity generation industry is characterized by priceinelastic demand, significant short-run capacity constraints and extremely costly storage. Under such circumstance, even when the overall market HHI measure is low, firms with small market shares can still excise market power.

\subsubsection{Lerner Index}

Another approach that is commonly used in measuring the abuse of market power uses the Lernex index. It has the following form:

$$
L=\frac{P-M C}{P}
$$


where $P$ represents actual market price and $M C$ marginal cost. The Lerner index quantifies the extent to which that supplier is able to raise the price above its marginal costs of supplying the last unit of a good before reaching equilibrium. Under perfect competition where the firms have no market power, in equilibrium they will set their price equal to the marginal cost, therefore yield a Lerner index of 0 . A positive Lerner index suggests supplier's ability to charge a price above their marginal cost and as their market power increases, the index increases.

The Lerner index can also be represented as the negative inverse of price elasticity of demand evaluated at the market-clearing price. In contrast to the HHI measure, the Lerner index therefore does take into account consumer's responsiveness toward a change in price (that is, the price elasticity of demand).

There are a number of regulatory agencies that adopt this approach. The DOJ of USA sets the threshold at $5 \%$ such that a firm will be considered abusing market power if $\mathrm{P}$ exceeds $\mathrm{MC}$ by more than $5 \%$. However, the difficulty in the implementation of the Lerner index is that it is very difficult to determine marginal cost since the supplier production cost information is generally confidential and marginal cost must be estimated. A second major difficulty with the Lerner condition is that price equals marginal cost is efficient - thereby suggesting pricing without market power - only when marginal cost is long run marginal cost. ${ }^{27}$ In the short-run, price greater than marginal cost is required to meet any fixed costs if the firms are to be viable. In industries, such as the electricity industry, with large irreversible investments price greater than shortrun marginal cost will occur even when the industry is very competitive.

\footnotetext{
${ }^{27}$ Long run marginal cost being the cost of expanding firm capacity by one unit and producing at the new capacity.
} 
Although an accurate measure of marginal cost for electricity production is hard to calculate, in the past 20 years there has been a number of empirical studies that have developed approaches to modelling the marginal cost function. Three popular approaches will be summarized in the following section. They are:

(1) Simulation analysis at market level: Borenstein et al (2002)

(2) Cournot Equilibrium simulation approach: Bushnell and Borenstein (1998)

(3) Estimating marginal cost function using observed bid data: Wolak (2003a)

\subsubsection{Simulation analysis}

One popular approach in assessing the actual abuse of market power in electricity markets is called simulation analysis. This approach attempts to simulate a market level marginal cost curve of generation as if all the generators are working in a hypothetical competitive market. By using information collected on the technical characteristics of the existing generation plants present in the market, the market marginal cost curve for that trading period is constructed by stacking generators from least expensive to most expensive.

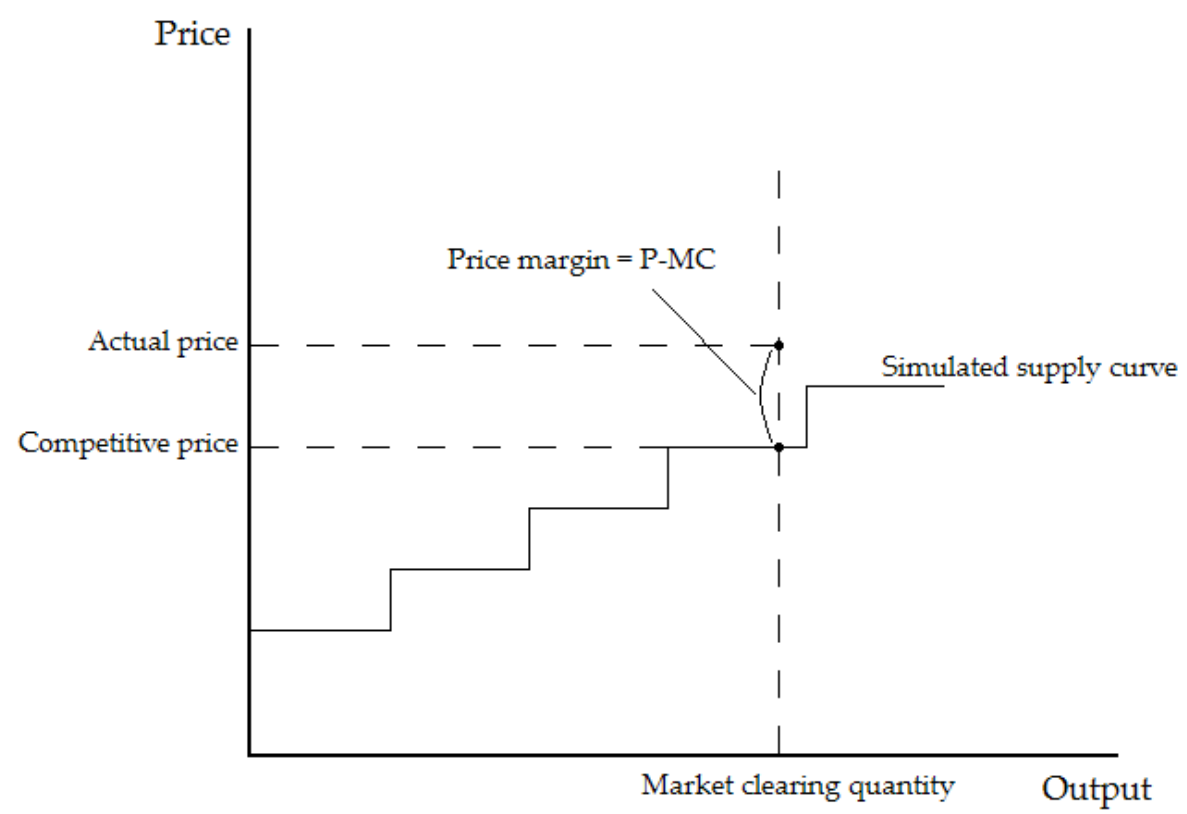

Diagram 7-1: Demand and supply in the electricity market 
Given the market clearing quantity, the corresponding cost on the simulated market marginal cost curve gives the competitive benchmark price. The markup in this market for this time period is calculated by comparing the competitive benchmark price with the observed market-clearing price. The extent to which the latter price is in excess of the competitive cost (the mark-up) gives an indication of the amount of market power being exercised in this market.

This approach was initially developed by Wolfram (1999) in her study of the British electricity spot market. The same approach has been applied in the California market in a number of studies: Borenstein et al (2002) applied this approach in measuring the market inefficiencies in the California electricity spot market using confidential data on costs from CAISO for the period from June 1998 to October 2000; Joskow and Kahn (2002) conduct the same analysis but relying on data which is generally available to the public. Other studies include Wolak, Nordhaus and Shapiro(2000) and Hildebrandt(2000).

In Borenstein et al 2002 (BBW(2002)), simulation analysis was applied to analyze market inefficiency in the California electricity spot market using data from CAISO for the period June 1998 to October 2000. However, the California market is more complicated than others as quantity supplied in California wholesale market consists of not only the amount generated by the in-state generators but also the amount that is generated by generators outside California. BBW(2002) deal with this problem by taking the imports away from the total demand function to obtain the residual demand of in-state generation while simulating the marginal cost curve for in-state generation. Competitive price benchmark price is calculated by finding the intersection of the in-state residual demand curve, net of imports, and the simulated marginal cost curve. 
BBW estimated the residual market demand to be met by in-state fossil-fuel units $q_{f f}^{t}$ in the following way:

$$
q_{f f}^{t}=q_{t o t}^{t}+q_{r e g}^{t}-q_{m t}^{t}-q_{r s v}^{t}-\left(q_{i m p}^{t}+\Delta q_{i m p}^{t}(p)\right)
$$

where $q_{\text {tot }}^{t}$ is the observed ISO generation including output generated by all generation units and imports. The quantity $q_{r e g}^{t}$ represents the addition to demand due to the need for capacity dedicated to regulation reserve. $q_{m t}^{t}$ and $q_{r s v}^{t}$ are the amount of energy generated by must-take generation and by reservoir generation. The must-take units are the units that always operate under a regulatory side agreement, for example nuclear generation. The reservoir generation include hydro generation and geothermal generation. Instead of subtracting away the observed amount of import $q_{i m p}^{t}$, an adjustment term $\Delta q_{i m p}^{t}$ is also taken away from the total output. This adjustment term is to capture the responsiveness of imports to price. Notice that if generators outside the California market were perfectly competitive, then their marginal cost of producing the very last unit of $q_{i m p}^{t}$ would equal the market clearing price in the overall California market and this price would be a cap on the price in that market. For the in-state generators to effectively exercise market power (that is, to push the market price above the competitive level), they must withdraw their own generation and substitute it with more expensive imports. Therefore when the price is higher than the competitive level the observed imports will be higher than the level obtained when price is lower than the competitive level. In BBW, data on the outside suppliers' adjustment bid is used to adjust imports to the California price.

For this reason, though the aggregate demand faced by the entire market is inelastic, after adding back the capacity dedicated to regulation reserve, and taking away the quantity generated by other in-state non-fossil-fuel generations 
and the adjusted net imports that reflect its competitive responsiveness to price, the residual demand faced by in-state fossil-fuel generators becomes elastic to the extent that imports are elastically supplied.

To estimate the market level marginal cost of production for all in-state generators, they divide the total generation into three categories: reservoir (hydro, geothermal), must-take and fossil-fuel generation. The must-take units are the units that always operate under a regulatory side agreement, for example the nuclear generation. BBW(2002) also assumed that the actual observed output of reservoir resources is the output that would be produced by a price taking firm participating in a perfectly competitive market. It relies on CAISO settlement data for the hourly output of must-take generation, geothermal and hydro production at the market-clearing point. The residual demand of in-state generation beyond the sum of the output produced by these two generation categories above will be served by using the fossil-fuel generation. In sum, BBW(2002) calculate the marginal cost of satisfying the remaining demand with the state's fossil-fuel resources, where fossil fuel generators face responsiveness in demand cause by price sensitive out of state supplies.

For fossil-fuel generators, the marginal cost is estimated using the fuel costs and heat rate of each generating unit and the variable operating and maintenance cost of each unit. The cost of NOx emission credit is also taken into account. BBW also used Monte Carlo simulation to model the unplanned outage of each generating unit. For each hour, they make 100 fossil-fuel-generation marginal cost curve estimates, each reflecting a combination of independent Monte Carlo draws for the forced outages of each generation unit from the outage distribution.

Given the 100 simulated marginal cost curves for the in-state fossil-fuel generation in hourly trading periods, and the residual demand faced by those 
generators after taking away the quantity generated by the other two classes of in-state generators and imports, they find the intersection of the residual market demand curve with each one of the 100 simulated marginal cost curves. An estimate of the competitive benchmark price in that hour that assumes pricetaking behaviour is calculated by taking the average of the demand intersections with the 100 estimated marginal costs.

By comparing this estimated competitive benchmark price with the observed market clearing price, Borenstein(2002) found that from June 1998 to October 2000, the observed market clearing price in the California electricity market significantly departed from competitive pricing during high demand periods.

It is very difficult to measure the variable per unit cost of electricity generation. However, in many electricity markets, before the structural reform in the 1990s, the entire market demand was served by one state-owned generator. Due its history of regulation, data existed on the hourly output for all generating units, transmission power flows and some technical characteristics of each generation plants, for example the fuel cost, efficiency rate of generation, capacity constraints etc. Given the availability of those data, the estimation of generating unit-level variable costs can be done quite accurately. This is the case for many countries (for example, United Kingdom, United States), but not for New Zealand.

However, there are a few shortcomings of this approach. One big weakness is that a lot of major simplifications must be made in order to simulate the market competitive supply curve. One example of these simplifications is that $\mathrm{BBW}(2002)$ assumed that there is no transmission congestion constraint, no transmission losses and zero transmission cost. Ignoring these constraints and costs, may cause underestimation of the marginal cost. By doing this, they generally do not incorporate the start up costs or the minimum load effects. 
Working in a static setting causes problems in estimating the marginal costs in hydroelectric generation. For example in BBW, they assumed that hydro generators are competing competitively and they use the observed production of hydro resources for each hour (which is exogenous) to represents the amount that will be generated by the hydro generators in that period. They did not take into account the dynamic features of hydro generators' behaviour. This is because in a dynamic setting, the marginal cost of water is not just the direct cost of turning water into electricity; it should also reflect the opportunity cost of storing hydro energy and using it at some later time. Therefore modelling the hydro generation in a static setting may cause an under-estimation of its marginal cost. The same issues apply to gas-fired generation (see Evans and Guthrie (2009)). It means that the model of BBW does not include decision making in the market that recognises environmental uncertainties of that market.

However, the approach used in BBW could be modified to estimate the market competitive benchmark price of a market with hydro as long as the import only accounts for a very small proportion of total energy generation, and there was no volatility in the gas price or availability. BBW, because it had limited information on the marginal cost of imports could not accurately simulate a marginal cost curve that accounts for both fossil-fuel generation and imports. Instead they subtract the quantity supplied by the out-of-state generators from aggregate demand using the bidding information on imports, to produce an estimate of the residual demand faced by the in-state generators. It then calculates the competitive price by finding the intersection between this residual demand curve and the simulated marginal cost curve of in-state fossil generations (this is the part where they have more accurate information on the costs and efficiency rates). For a country with significant hydro generation and fossil-fuel generation, the BBW approach is problematic because the behaviour of hydro generators matters and it is impossible to calculate the marginal cost of hydro in a static setting. This is a consequence of the fact that the hydro marginal 
cost reflects the option value of water and this option value cannot be calculated in a static model since it requires assessment of the value of future generation. In a closed economy, with sufficient information on the bid/offer for the hydroelectric energy, then the way BBW treat the import can be adopted here to adjust for the hydro generation: that is the marginal cost of gas-fired generators can be compared to demand for their generation where the demand depends upon the amount and price responsiveness of hydro generation. However, feasibility of adopting this approach depends on the availability of those bid/offer information and whether there is adequate gas-fired capacity and no gas price or availability issue. It is likely to work well if hydro generation only accounts for a small percentage in the total market generation and there are no gas, NOx, storage constraints or frictions.

\subsubsection{Cournot Simulation method}

The Cournot ${ }^{28}$ simulation method is similar to the simulation analysis above in that both construct the marginal cost curve for a trading period by stacking generators/generation units from least to most expensive. The difference between the two is that, in the simulation analysis of BBW the market as a whole is considered ignoring the strategic behaviour of generators within the market, whereas in the Cournot simulation method it is assumed that the generators within an oligopoly market compete with each other under Cournot competition. That is each generator will choose the quantity of generation by acting as a monopolist on their residual demand curves, holding fixed the quantities other competitors are generating.

The Cournot Equilibrium analysis was first developed by Klemperer and Meyer (1989). Since then this approach has been frequently applied in analyzing market

${ }^{28}$ Recherches (1838) 
power in the UK electricity market and markets elsewhere. For example, Bolle (1992), Green and Newbery (1992) and Green (1996) used this approach to model the UK electricity market. The reasons for its popularity are first that the majority of electricity markets worldwide have an oligopoly structure; and second, the model explicitly models the strategic behaviour of interacting suppliers, but in a particular way.

In 1998, Borenstein and Bushnell applied it to analyze the potential for market power in California's electricity industry. The algorithm of the Cournot simulation method used in Borenstein and Bushnell (1998) is as follows:

- First, simulate the marginal cost functions for each generator that they assume acts as a Cournot competitor as for the BBW approach, but for each generator they simulate its marginal cost curve by stacking the generation units owned by this firm from least expensive to most expensive. They use the observed data on must-take generation and hydro generation to calculate the amount of non-fossil-fuel generation that they are willing to supply. It is assumed that the remaining demand is satisfied by using the state's fossil-fuel resources. They construct the marginal cost functions of fossil-fuel generation for each Cournot generator using the plant heat-rate and operating cost data obtained from the California Energy Commission and other sources.

- Using the same approach as BBW, Bushnell and Borenstein assume that quantities produced by the out-state generators are priced at a competitive price. Therefore the residual demand curve that is faced by all Cournot fossil-fuel generators in the market equals the market aggregate demand minus the amounts of must-take generation, hydro and competitive imports. Further, each generator (Cournot player) in the market faces its residual demand function that is the aggregate demand 
faced by all in-state fossil-fuel generators minus the quantities being produced by all the other in-state generators.

Using the marginal cost functions of Cournot competitors and their residual demand functions, BB used a grid-search method to calculate the Cournot equilibrium iteratively. The first Cournot player sets output competitively using its simulated marginal cost function while assuming that the other players have no outputs. The second firm sets its output competitively under the assumption that the first firm will maintain the output at the level that was calculated in the previous iteration, and the output from all the other players remain as zero. Then the third firm, taking the quantities calculated in the previous two calculations as given, sets the output monopolistically given the residual demand it faces, and so on. The process repeats, returning to each supplier with each re-setting their output monopolistically using the most recent output decision of the others, until the output levels do not change with iterations. The price at that supply-equilibrium corresponds to the benchmark price in a competitive oligopoly market where all players play one-shot Cournot.

The treatment Bushnell and Borenstein (1998) used in calculating the hydro generated (demand) quantity is different from the approach adopted in BBW. Instead of using the observed quantity of hydro generation, they use a technique known as "peak-shaving" to approximate a Cournot competitor's optimal allocation of hydroelectric energy. That is, they allocate the available hydroelectric production in a month across hours of the month by allocating hydro to the highest demand periods subject to minimum and maximum flow constraints. The reason for doing this is to capture the property that hydro generation has the ability to allocate its resources over time. The producers would always want to move hydro output to the time period in which the marginal revenue was the highest. Since marginal revenue is not observable, they instead allocate the hydro output to the time period when the demand is 
the highest. If demand perfectly correlates with the marginal revenue then this allocation reflects suppliers' optimal behaviour for hydro allocation. However, if demand is imperfectly correlated with the marginal revenue, the approach could overstate or understate of the exercise of market power.

\subsubsection{Estimating marginal cost function using observed bids: Wolak(2001)}

Rosse (1970) estimated the underlying marginal cost of monopolists by using a sample of monopoly local newspapers and the assumption of profit maximization. After that, similar research has been done by empirical industrial organization economists that devise estimation procedures to recover cost functions from data on market-clearing prices and quantities.

The basic idea of all these techniques is as followed:

Let $P(q, W, \theta, \epsilon)$ denote the inverse demand function facing a monopolist, and let $C(q, Z, \theta, \eta)$ be its total cost function. $q$ represents the quantity, $W$ and $Z$ are the demand and supply shifters respectively. $\theta$ is the vector of parameters to be estimated, and $\epsilon, \eta$ represents the stochastic shocks that are not observed.

For the monopolist, its profit function is

$$
\pi(q)=P(q, W, \theta, \epsilon)-C(q, Z, \theta, \eta)
$$

When the profit is maximized, the following condition has to hold:

$$
\pi^{\prime}(q)=P^{\prime}(q, W, \theta, \epsilon) q+P(q, W, \theta, \epsilon)-C^{\prime}(q, Z, \theta, \eta)=0
$$

That is at equilibrium, quantity $q=q^{E}$, such that

$$
\pi^{\prime}\left(q^{E}\right)=P^{\prime}\left(q^{E}, W, \theta, \epsilon\right) q^{E}+P\left(q^{E}, W, \theta, \epsilon\right)-C^{\prime}\left(q^{E}, Z, \theta, \eta\right)=0
$$


Suppose that the inverse demand faced by the monopolist takes the form of $P(q, W, \theta, \epsilon)=a+b q+c W+\epsilon$, where $a, b, c$ are the elements of vector $\theta$, then the profit maximization condition becomes equivalent to the following:

$$
C^{\prime}\left(q^{E}, Z, \theta, \eta\right)=P^{\prime}\left(q^{E}, W, \theta, \epsilon\right) q^{E}+P\left(q^{E}, W, \theta, \epsilon\right)=b q^{E}+p^{E}
$$

The econometrician can use the instrumental variable technique to estimate the value of $a, b$ and $c$. Given the estimated values for these parameters, together with the market-clearing prices and quantities, the econometrician can solve for the value of marginal $\operatorname{cost} C^{\prime}\left(q^{E}, Z, \theta, \eta\right)$.

Once an estimate of marginal cost is obtained, it is substituted into the formula of the Lerner Index to get an estimate of the amount of market power possessed by this firm.

Wolak (2001) adopted this concept in his paper, extended it and presented several techniques to estimate the underlying marginal cost function in a bidbased electricity market using data on bids and market clearing prices and quantities from that market.

This approach is not very useful to our analysis, because the model will not give us any information on bids submitted by the generators in each period.

However, Wolak(2003) uses yet another approach. It measures the market power at firm-level for each of the five largest electricity suppliers in California in the state's real-time market for the period from 1 June to 20 September of 1998, 1999 and 2000. In this paper, Wolak measures the level of market power abuse for firm $\mathrm{j}$ in hour $\mathrm{h}$ by evaluating the value of the Lerner index at the marketclearing prices and quantities. Instead of taking the ratio between mark-up and market price, Wolak calculates this index by equating it to the price elasticity of demand evaluated at market price for firm $j$. Wolak demonstrates in the first half 
of the paper that when a firm is bidding according to its expected profit maximizing bid curve $S(p)$ where $S(p)$ is the function passing through all of the ex-post profit maximizing price and quantity pairs associated with all possible residual demand curve realizations, the ratio of the mark-up (P-MC) to market clearing price equals the negative inverse of the price elasticity of demand evaluated at this market clearing price. That is the following equation holds for each hour of the day $h$, and each supplier $\mathrm{j}$ :

$$
\frac{P_{h}-M C_{j h}}{P_{h}}=-1 / \epsilon_{h j}
$$

where $P_{h}$ stands for the market clearing price at hour h; $M C_{j h}$ is the marginal cost of producing the last unit before reaching market clearing quantity for firm $\mathrm{j}$ in hour $\mathrm{h}$, and $\epsilon_{h j}$ is the price elasticity of firm j's residual demand evaluated at equilibrium.

Wolak(2003) argues that in the CAISO real-time market, due to the flexibility in how the bid curves of a market participant can vary across hours of the day, generally the bids submitted by generators would not deviate much from their optimal bidding function that satisfies equation [47].

Given that this condition holds, the Lerner index is calculated in the following steps: first, computes the aggregate demand for electricity in the CAISO's realtime market. Second construct the aggregate demand function by ranking consumer's demand bid up from the highest bid to the lowest. Thirdly, derive firm $j$ 's residual demand by subtracting the total amount supplied at this price by all market participants other than firm $\mathrm{j}$ given their optimal bidding function. Fourthly, compute the slope of firm j's residual demand at market clearing price by using a first difference approximation. That is, find the closest price above $P_{h}$ such that the residual demand is less than the value at $P_{h}$, call it $P_{h}(l o w)$; then 
find the closest price below $P_{h}$ such that the residual demand is higher than value $P_{h}$, call it $P_{h}(h i g h)$. Finally approximate the price elasticity of residual demand of firm $\mathrm{j}$ in hour $\mathrm{h}$ by the slope of the line that passes through those two points. That is $\epsilon_{j h}$ can be computed as

$$
\epsilon_{j h}=\frac{D R_{j h}\left(P_{h}(\text { high })\right)-D R_{j h}\left(P_{h}(\text { low })\right)}{P_{h}(\text { hight })-P h(\text { low })} \times \frac{P_{h}(\text { high })+P_{h}(\text { low })}{D R_{j h}\left(P_{h}(\text { hight })\right)+D R_{j h}\left(P_{h}(\text { low })\right)}
$$

Using this approach, Wolak(2003) calculates an approximate value for the hourly price elasticity of residual demand for each firm evaluated at its market clearing price in that hour, then takes the negative inverse to find out the value of the Lerner index for that firm in that hour. Wolak(2003) then takes the average Lerner index across time. The average hourly value of $L_{j h}$ for each supplier for the period from 1 June to 30 September gives an annual measure of the amount of unilateral market power possessed by firm $\mathrm{j}$.

Using this approach, Wolak (2003) found that the average hourly Lerner indexes are significantly higher in 2000 relative to 1998 and 1999, for all firms in the California real-time electricity market. Wolak(2003) concludes that all firms exercised more market power in 2000 compared to the prior 2 years. The means for the hourly Lerner index for the months in 1998 are statistically significantly greater than the means for 1999. However, within a particular year, the Lerner index for one firm is not statistically different from the Lerner index of the other and this statement holds true for all three years. 


\subsection{Approaches to measure market power in the New Zealand Electricity Market}

In the following section, I will discuss the approaches that can be used to assess the performance of market power tests in the New Zealand electricity market.

The model presented in this paper improves the literature that models the amount of hydro electricity generation in a static setting as it takes into account the option value of water (which arises from a generator's ability to allocate hydro resources efficiently across time) and solves for the equilibrium in a dynamic setting. Given a simulated series of water inflow $y_{t}$ that is generated using Monte Carlo simulation, for time $t$ where $t$ goes from 0 to $n$, we have the following information:

\begin{tabular}{|l|c|c|}
\hline & Competitive Market & Monopoly \\
\hline Market clearing prices & $P_{c t}$ & $P_{m t}$ \\
\hline Hydro generation & $Z_{c t}$ & $Z_{m t}$ \\
\hline Gas generation & $M_{c t}$ & $M_{m t}$ \\
\hline Storage lake level & $S_{c t}$ & $S_{m t}$ \\
\hline Shadow price of water & $W_{s_{c t}}$ & $W_{s_{c t}}$ \\
\hline
\end{tabular}

At the end of each trading day, the public can observe the market clearing price $P_{t}$, the amount of hydro generation $Z_{t}$ and the amount of gas generation $M_{t}$. This information is generally publicly available through the Electricity Commission. The commission also has some data available on the storage lake level $S_{t}$. The marginal cost of certain generation plants, for example the gas plant, is likely to be estimated using historical data (for example, in New Zealand, the Ministry of Economic Development has some data on the marginal 
cost of different types of electricity generation plants), similarly for the transmission costs. However, the data on the shadow price of water $W_{s t}$ are generally not publicly available.

To conduct the assessment on the performance of market power tests in the New Zealand electricity market, I run a chosen market power test over the results given by the simulations. The party that runs the market power test can only use the data that are publicly available, in our case the market clearing prices and quantities. The chosen market power test is effective if it can distinguish the data generated in a competitive market setting from the data generated in the case assuming the market is monopolistic.

The market power test that is described in Wolak (2003b) which estimates a firm's price elasticity of residual demand at the market clearing price cannot be applied to our model. This is because we do not have any information on consumer's demand bids and on offer bids submitted by the generators. Without this information, it is impossible to construct firms' residual demand in which the price elasticity of demand can be calculated from. Similarly, the method described by Wolak (2003a) cannot be used on the data that we have as this method also requires information on generators' bids which we lack.

The market power test that we adopt in this paper is a simplified version of the one that is described in BBW. The essence of their approach is to simulate the marginal cost curve for the fossil-fuel generator, then compare the observed market clearing price for the overall market with the marginal cost of fossil-fuel generation at its quantity produced. However, the market power test that we use here is much simpler than the one that is described in their paper. This is because in our model there are only two types of generation plants, the hydro plant and the gas plant. The overall market demand is entirely served by those two plants without any imports. Therefore there is no need for us to adjust for 
quantities produced by the must-take generations (nuclear) and for the imports in the way that is done in $\operatorname{BBW}(2002)$.

Assume that the party that runs the market power test can get access to data that enable them to obtain a close estimate on the cost of gas generation $C(m)$ and the transaction costs $\left(k_{1}, k_{2}\right)$. Using those data they are able to simulate the marginal cost curve for the gas plant. For each given quantity of gas generation $\mathrm{m}$, the marginal cost of gas generated electricity at this quantity equals the marginal cost of producing this unit of electricity using gas plant $\left(C^{\prime}(m)\right)$ plus the cost of transmitting it to the end-user $(\mathrm{k} 2)$. At the market clearing price, the quantity of demand faced by the gas generator equals the equilibrium quantity of gas generation, which also equals the total market clearing quantity minus the amount of hydro generation. We can use the simulated marginal cost curve for gas generation to find out the marginal cost of gas generation at the observed quantity of gas generation. The difference between the marginal cost and the observed market-clearing price gives us some indication of the amount of market power being exercised in this market.

We can run this test on the data generated in the competitive market setting and also on the data generated assuming the market is monopolistic, and then compare the gap between price and marginal cost in one case with the other. If the gap calculated in the monopolistic market is significantly bigger than the gap calculated in the competitive market, it suggests that this market power test can effectively distinguish between the market outcomes from a competitive market and from a monopolistic market.

This approach has been widely used in detecting the existence of market power abuse in many electricity markets. However, it only works under the assumption that the gas generation always serves as the marginal generator. Problems occur 
when the gas generation is no longer the marginal generator, for example if there is an upper bound on the gas generation capacity and the market clearing quantity is higher than this upper limit. This is because if this scenario occurs, the marginal cost of gas generation at the equilibrium quantity of gas generated electricity, which equals its maximum capacity, fails to represent the overall marginal cost of generation at the market clearing quantity. The overall marginal cost equals the cost of producing the last unit of electricity, which is generated using hydro. In that case using the marginal cost of gas generation as an estimate of the overall marginal cost would cause downwards bias.

In this section, we are interested in investigating the effectiveness of this market power test in the New Zealand electricity market. Different from other electricity markets, in New Zealand hydro generation accounts for a large proportion in the total electricity production. With abundant amount of water resources, large water storage capacity, strict rules on the carbon emission and capacity constraint on the gas generation plant, it is likely that gas generation will not always run as the marginal generator therefore as a result these properties could potentially affect the effectiveness of this market power test. If this test fails to distinguish between the prices and quantities generated under perfect competition and under monopoly, then we can conclude that this widely adopted approach is not suitable for assessing the exercise of market power in the New Zealand electricity market.

Theoretically, there is another approach that can be used to test the existence of market power in a market where the hydro generation serves as the marginal generator. This simplified version of market power test described in BBW can be modified in the way that instead of subtracting off the hydro generation, subtract the gas generation. One can simulate the marginal cost curve of hydro generation, then compare the marginal cost of generation at the market clearing quantity for hydro generation with the market clearing price. However, this 
approach is not implementable as it is very difficult to estimate the marginal cost of water using publicly available data. This is because the choice of the optimal level of hydro generation depends on a number of factors, for example the lake level, inflow and future electricity prices etc and therefore the marginal cost curve of hydro generation cannot be simulated without knowledge on those factors.

\subsection{Results}

Using the market power test we described in section 7.2 on the data generated in the standard model we find that the market power test can successfully distinguish between a market with and without market power.

First we run the market power test described in section 7.2 on the data generated in the standard competitive setting. Diagram 7-2 below shows the difference between the market clearing price of electricity and the per unit marginal cost of generating and transmitting gas generated electricity at the equilibrium quantity of gas generation. By comparing the price and the marginal cost we find that in the competitive setting, the sum of marginal generation cost and transmission cost of gas generated electricity is fully reflected by the market clearing price in the competitive market - that is, there is no difference between the price and the marginal cost of gas generated electricity over the 30 years range in the competitive market setting. These results suggest that the market power test we adopted here does not find existence of any abuse of market power in the data simulated in our standard competitive model, as expected. 


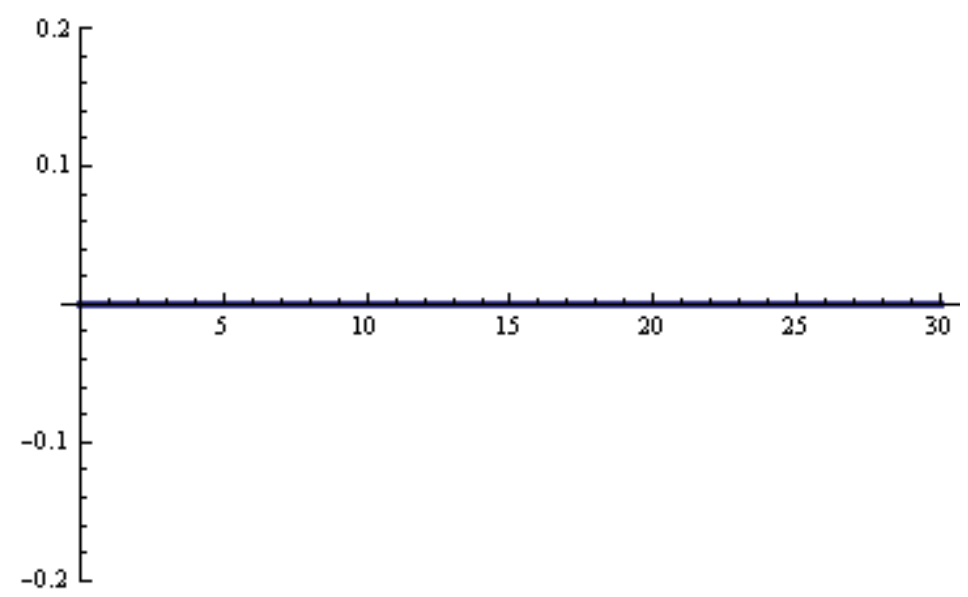

Diagram 7-2: Difference between the price and the marginal cost of gas generation $\left(P\left(m^{*}+z^{*}\right)-M C\left(m^{*}\right)-k_{2}\right)$ in the standard competitive market.

Applying the same market power test method on the data generated in the standard pure monopoly setting, we find strong evidence of the existence of market power abuse in this market. Diagram 7-3 presents the difference between the market clearing price and the marginal cost of gas generation (which includes both the marginal generation cost and the transmission cost) at the equilibrium quantity of gas generation on a daily basis over 30 years time. We observe that the price is consistently higher than the marginal cost of gas generation. The mark-up (price minus the marginal cost) is especially higher in periods when the inflow is low.

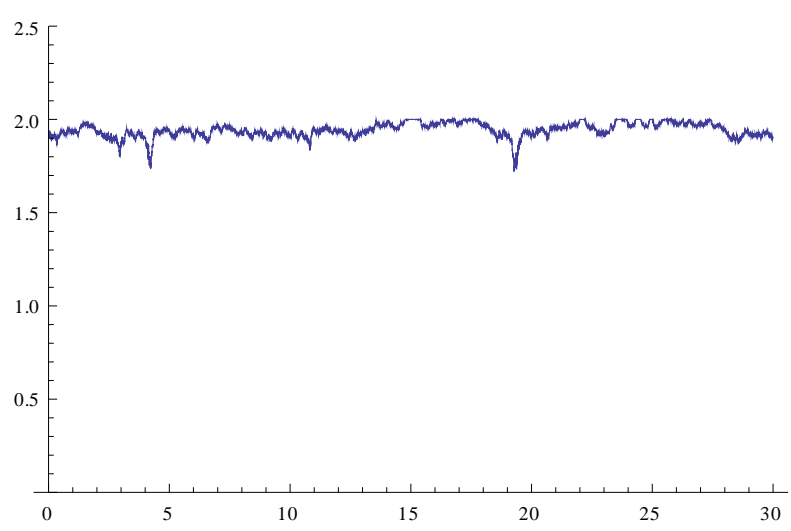

Diagram 7-3: Difference between the price and the marginal cost of gas generation $\left(P\left(m^{*}+z^{*}\right)-M C\left(m^{*}\right)-k_{2}\right)$ in the standard monopoly market. 
The market power test helps us to clearly distinguish the market with and without the abuse of market power - we observe no difference between the price and the marginal cost of gas generated electricity at the equilibrium quantity in the competitive market, and a significant difference in the market with the monopoly power. This test method also works on a number of other settings with changes in some of the input parameters, for example the ones we have seen in the previous section.

In most of the cases we observe that the market clearing price equals the marginal cost of gas generated electricity in the data generated in the competitive setting and the price exceeds the marginal cost of gas generated electricity in the data generated in the monopoly market.

However, we do find that this method fails to distinguish the data generated in a competitive market and in a monopoly market in certain cases which includes the cases when only 40 percent of the gas generation capacity in the standard setting is available and when the marginal cost of gas generation is 60 percent lower than the standard setting.

In both cases we observe that the price exceeds the marginal cost of gas generated electricity not only in the data generated in the monopoly market but also in the data generated in the competitive market as presented in diagrams 74 and 7-5. Though by comparing the difference between price and marginal cost we can tell that the data generated in the competitive setting has a significantly lesser extent of market power abuse than the data generated in the monopoly setting, as the price in the competitive market exceeds the marginal cost only in some periods but not all, and the difference is also much smaller than they are in the monopoly market setting. However, the test cannot give us any clear indication on in which market the existence of market power abuse is in absent; 
instead it reports the existence of market power abuse even when it is applied to data that is generated in a perfect competitive setting.

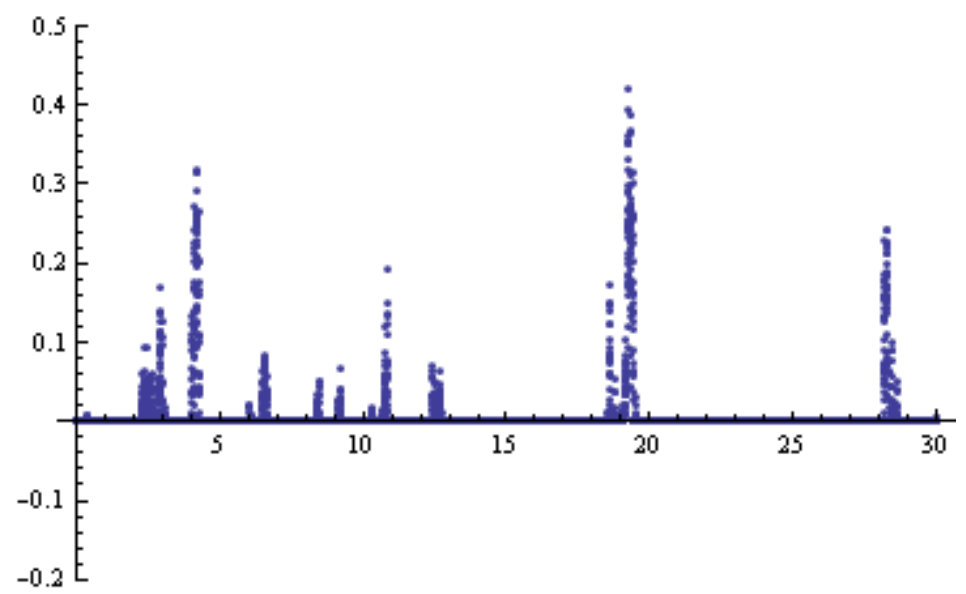

Diagram 7-4: Difference between price and the marginal cost of gas generation (including the transmission cost) in a competitive market when the capacity of gas generation is small.

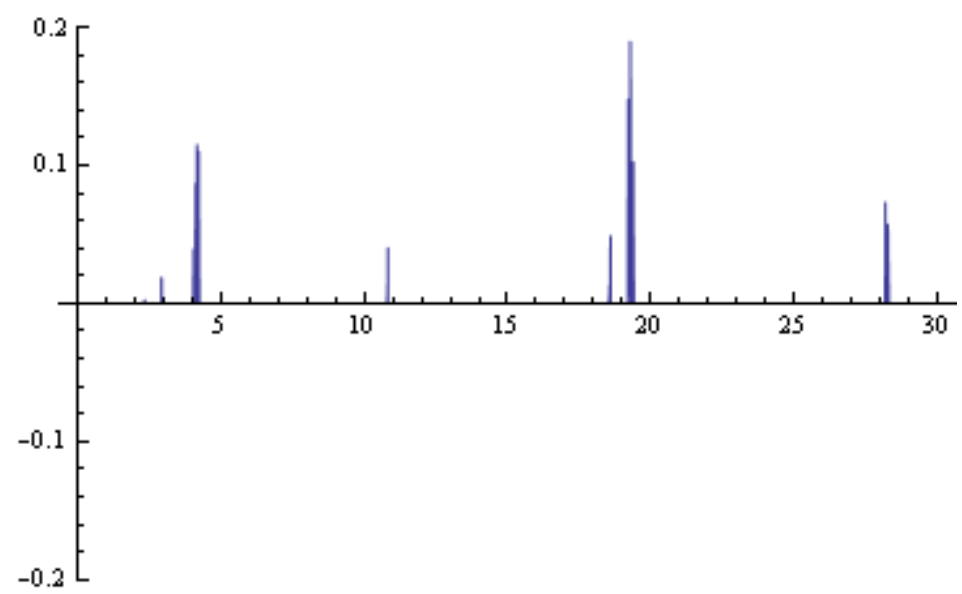

Diagram 7-5: Difference between price and the marginal cost of gas generation (including the transmission cost) in a competitive market when the marginal cost of gas is low.

The reason behind the failure of the market power test is that in both of these cases, the gas generation, in some periods, no longer serves as the marginal generator in the market. Instead in those periods it reaches its maximum generation capacity allowing the price to exceed the marginal cost. In the case 
with small maximum generation capacity, the full capacity is so small that it is easily reached in periods when the inflows are low; and in the case with low marginal cost coefficient, the cost of gas generation is low so in some periods the social planner, especially when the shadow price of water is high, is happy to use as much of the gas capacity as it can due to its low operational marginal cost. In these periods where the gas generation reaches its maximum capacity, the marginal cost of gas generation at the equilibrium quantity of gas generated electricity (which equals to its maximum capacity) fails to represent the overall marginal cost of generation at the market clearing quantity. The overall marginal cost equals the cost of producing the last unit of electricity, which is generated using hydro. Using the marginal cost of gas generation as an estimate of the overall marginal cost would cause a downwards bias. As a result the positive difference between the market clearing price and the marginal cost of gas generation may not be evidence of the existence of market power; it is likely that a positive difference will be observed in some periods even when the data come from a perfectly competitive market.

In the actual New Zealand market, some other non-hydro generation in New Zealand also faces reservoir constraints due to the inefficiency in the speed of delivery of the reserves of fuel ${ }^{29}$. Accordingly, much of the analysis of hydro generation in this thesis can be applied to non-hydro generation in New Zealand.

${ }^{29}$ A good example of this is thermal generation. 


\section{Section 8 Conclusion}

This paper introduces a tractable approach to model a hydro dominated electricity market. This model properly accounts for the key characteristics of such markets, that fuel availability is stochastic and that there exists the possibility of managing this volatility by means of storage. The model permits study of cases where there is a mix of generators with different fuels. It enables us to compute the equilibrium outcomes and the endogenous hidden marginal cost of water, or in other words the forward-looking shadow price of water, under different market structures. The model is calibrated to the New Zealand electricity market, taking into account the property of hydro dominance, volatile inflow and low capacity of lake storage.

We find that with the ability to store the fuel the price and quantity in the market are volatile, but less volatile than the fuel availability. A competitive market produces higher welfare than the monopolist in each period, supplying a large quantity at lower prices. However, the monopolist uses significantly less gas than the social planner. Overall the monopolist has less volatile market outcomes. The model suggests that an increase in the mean of the hydro fuel availability lead to a significant increase in welfare. On the other hand a decrease in the ability to forecast inflows, resulting from less rapid mean reversion, reduces the average welfare.

Our paper also shows that the presence of real options embedded in the stored fuel make it difficult to detect abuses of market power in practice under certain circumstances - the price exceeds the operational marginal cost in a market that is perfectly competitive when the gas generation reaches its maximum capacity. This can happen when the marginal cost of gas is low and/or when the capacity of the gas plant is small enough. This suggests that the positive difference 
between the market clearing price and the marginal cost of gas generation estimated in a static setting may not be evidence of the existence of market power.

The approach is tractable enough such that it can be modified to fit other market structures, for example the oligopoly structure. The model can also be calibrated for other markets other than the New Zealand Electricity to model their markets.

An important extension would be to incorporate stochastic demand. This source of volatility and uncertainty is characteristic of electricity markets, and would likely affect the use of storage and the value attached to storage facilities. 


\section{Reference}

Bellman,R.E. 1957. Dynamic Programming. Princeton, NJ.

Borenstein, S., 2002, The trouble with electricity markets: understanding California's restructuring disaster, The Journal of Economic Perspectives, 16, 191-211.

Borenstein, Severen and James Bushnell, 1999, An Empirical Analysis of the Potential for Market Power in California's Electricity Industry, The Journal of Industrial Economics, 47(3),285-323.

Borenstein, Severin, James B. Bushnell, and Frank A. Wolak. 2002. Measuring market inefficiencies in California's restructured wholesale electricity market. American Economic Review 92:1376-1405.

Borenstein, S. and Bushnell, J. and Knittel, C.,1999, power in electricity markets: Beyond concentration measures, ENERGY JOURNAL-CAMBRIDGE, 20, 65-88.

Evans, Lewis, and Graeme Guthrie, 2009. "How Options Provided by Storage Affect Electricity Prices", Southern Economic Journal, 75(3), 681-702.

Evans, Lewis, and Richard Meade, 2005. Alternating Currents or CounterRevolution?. Victoria University Press. pp. 138.

Green, Richard, and David Newbery. 1992. Competition in the British electricity spot market. Journal of Political Economy 100:929-53.

Hildebrandt, Eric. ,2001, Further Analysis of the Exercise and Cost Impacts of Market Power in California's Wholesale Electricity Market, Report from the California Independent System Operator's Department of Market Analysis.

Hogan, Read and Ring. 1996. Using mathematical programming for electricity spot pricing. International Transactions in Operational Research, 3, 209-221.

Hortacsu, A and S.L Pullar (2008), 2005, Testing Models of Strategic Bidding in Auction: A Case Study of the Texas Electricity Spot Market. NBER Working Paper 11123.

Hortacsu, A and S.L Pullar (2008), Understanding stregic bidding in multiunit auctions: a case study of the Texas electricity spot market, The RAND Journal of Economics, 39, 86-114. 
Joskow, Paul L., and Edward Kahn. 2002. A quantitative analysis of pricing behavior in California's wholesale electricity market during summer 2000. Energy Journal 23:1-35.

Lyle, M. R. and R.J. Elliott 2009, A simple hybrid model for power derivatives, Energy Economics, 31, 757-767.

Rosse, J. N. 1970. Estimating Cost Function Parameters Without Using Cost Data., Econometrica 38:256-74.

Scott, T.J. and E.G. Read, 1996, Modelling Hydro Reservoir Operation in a Deregulated Electricity Market, Int. Trans. Opl Res, 3(3/4), 243-253.

Wolak, Frank A. 2001. Identification and estimation of cost functions using observed bid data: An application to electricity markets. NBER Working Paper 8191.

Wolak, Frank A. 2003. Measuring unilateral market power in wholesale electricity markets: The California market, 1998-2000. American Economic Review, Papers and Proceedings of the One Hundred and Fiftieth Annual Meeting 93: 425-430.

Wolak, F.A. and Nordhaus, R. and Shapiro, C., 2000, An Analysis of the June 2000 Price Spikes in the California ISO's Energy and Ancillary Services Markets, California ISO Market Surveillance Committee, Sep.

Wolfram, Catherine D. 1998. Strategic bidding in a multiunit auction: An empirical analysis of bids to supply electricity in England andWales. RAND Journal of Economics 29:703-725.

Wolfram, Catherine D. 1999. Measuring duopoly power in the British electricity spot market. American Economic Review, 89:805-826.

27 


\section{Appendix 1: Derivation of Hamilton-Jacobi-Bellman equation}

In section 1.1 we see that in a competitive market, given the value for $\mathrm{s}$ and $\mathrm{y}$, producer's objective is to choose the amount of hydro generation $\mathrm{z}$ and the amount of gas generation $\mathrm{m}$ at time $\mathrm{t}$ to maximize the expected present value of life time total surplus $\mathrm{W}$,

$$
W(s, y)=E_{0}\left[\int_{0}^{\infty} e^{-r t} \operatorname{TS}\left(z_{t}\left(s_{t}, y_{t}\right), m_{t}\left(s_{t}, y_{t}\right) ; y_{t}\right) d t \mid\left(s_{0}, y_{0}\right)=(s, y)\right]
$$

We can re-write this presentation of expected present value of life time total surplus as the summation of two parts: total surplus from the first period, and value of $\mathrm{W}$ one period later $W\left(s_{t}+d s, y_{t}+d y\right)$ discounted back one period,

$$
\begin{aligned}
& W\left(s_{t}, y_{t}\right) \\
& =T S\left(s_{t}, y_{t}\right) \cdot d t+e^{-r \cdot d t} E_{0}\left[W\left(s_{t}+d s, y_{t}+d y\right)\right] \\
& =T S\left(s_{t}, y_{t}\right) \cdot d t+(1-r \cdot d t) \cdot\left[W+E_{0}(d W)\right] \\
& =T S\left(s_{t}, y_{t}\right) \cdot d t+W+E_{0}(d W)-r W d t-r \cdot E_{0}(d W) \cdot d t
\end{aligned}
$$

The $\mathrm{W}$ on the left hand side of this equation cancel out with the $\mathrm{W}$ on the right hand side, therefore we are left with the following equation:

$$
0=T S\left(s_{t}, y_{t}\right) \cdot d t+E_{0}(d W)-r W d t-r \cdot E_{0}(d W) \cdot d t
$$

Given that $d y_{t}=v\left(y_{t}\right) d t+\phi\left(y_{t}\right) d \zeta_{t}$ and $d s_{t}=\left(y_{t}-z_{t}\right) d t$, we can present the expected present value of lifetime surplus at one period later in the following way, 


$$
\begin{aligned}
& W(s+d s, y+d y) \\
& =W(s, y)+\frac{\partial W}{\partial s} \cdot d s+\frac{\partial W}{\partial y} \cdot d y+\frac{1}{2} \frac{\partial W}{\partial s} d y^{2}+\cdots \\
& =W+\frac{\partial W}{\partial s}(y-z) d t+\frac{\partial W}{\partial y}\left(v \cdot d t+\phi \cdot d \varsigma_{t}\right)+\frac{1}{2} \frac{\partial^{2} W}{\partial y^{2}} \phi^{2} d t \\
& =W+\left[\frac{\partial W}{\partial s}(y-z) \cdot d t+\frac{\partial W}{\partial y} v+\frac{1}{2} \frac{\partial^{2} W}{\partial y^{2}} \phi^{2}\right] \cdot d t+\frac{\partial W}{\partial y} \cdot \phi \cdot d s_{t}
\end{aligned}
$$

That is,

$$
d W=W(s+d s, y+d y)-W=\left[\frac{\partial W}{\partial s}(y-z) \cdot d t+\frac{\partial W}{\partial y} v+\frac{1}{2} \frac{\partial^{2} W}{\partial y^{2}} \phi^{2}\right] \cdot d t+\frac{\partial W}{\partial y} \cdot \phi \cdot d \varsigma_{t}
$$

Knowing that $E\left(d \zeta_{t}\right)=0$,

$$
E(d W)=\left[\frac{\partial W}{\partial s}(y-z) \cdot d t+\frac{\partial W}{\partial y} v+\frac{1}{2} \frac{\partial^{2} W}{\partial y^{2}} \phi^{2}\right] \cdot d t
$$

Substitute the value of $E(d W)$ into the equation we obtained by re-writing $\mathrm{W}$, we get:

$$
\begin{aligned}
& 0=T S\left(s_{t}, y_{t}\right) \cdot d t+\left[\frac{\partial W}{\partial s}(y-z) \cdot d t+\frac{\partial W}{\partial y} v+\frac{1}{2} \frac{\partial^{2} W}{\partial y^{2}} \phi^{2}\right] \cdot d t-r W d t \\
& -r\left[\frac{\partial W}{\partial s}(y-z) \cdot d t+\frac{\partial W}{\partial y} v+\frac{1}{2} \frac{\partial^{2} W}{\partial y^{2}} \phi^{2}\right] \cdot d t^{2}
\end{aligned}
$$

Given that $d t^{2}=0$, by rearranging the equation above and dividing both side by $d t$, we obtain the Hamilton-Jacobi-Bellman Equation for the producer's optimization problem, that is,

$0=\underset{m, z}{\operatorname{Max}}(y-z) \frac{\partial W}{\partial s}+v \frac{\partial W}{\partial y}+\frac{1}{2} \phi^{2} \frac{\partial^{2} W}{\partial y^{2}}-r W+T S(m, z ; y)$ 


\section{Appendix 2: Initial guess for the generation policy}

The initial guess for the hydro generation policy has to be chosen specifically to ensure that the corresponding storage policy is feasible.

The generation policy that the social planner adopts together with the water inflow from the river in that period automatically determines the optimal storage policy. Specifically, for a particular level of inflow, the hydro generation policy will tell us how much water would be used to generate electricity. If the inflow is greater than the hydro generation $\left(y_{t}>z_{t}\right)$, then the social planner will put the unused water into the storage, the lake level will rise by $y_{t}-z_{t}$; whereas if the hydro generation $z_{t}$ given by the generation policy is higher than the inflow $y_{t}$, then social planner will take $z_{t}-y_{t}$ amount of water out of storage to be used to generate electricity.

In order to maximize the objective, it's more likely for the social planner to use less hydro to generate electricity if the shadow price of water $\left(W_{s}\right)$ is high. A High shadow price of water occurs when there's little water in the storage lake. Using less water in generation while having a big inflow will leave more to be stored in the lake. That is, when the storage level is low and the inflow is high, a reasonable hydro generation policy should choose to use low hydro in generation and to store more water. On the other hand, when there is plenty of water in the storage lake already and/or there's a small inflow from the river, the generation policy should tell the social planner to use a lot of water in electricity generation which makes it more likely to lower the lake.

This suggests that the initial guess of hydro generation policy should be a function of the inflow level and of the storage level and should have the following property: The level of change in storage $\left(y_{t}-z_{t}\right)$ following the initial generation policy should have positive sign when the storage is low and when 
the inflow is high; and it should have negative sign when the storage is high and when the inflow is low.

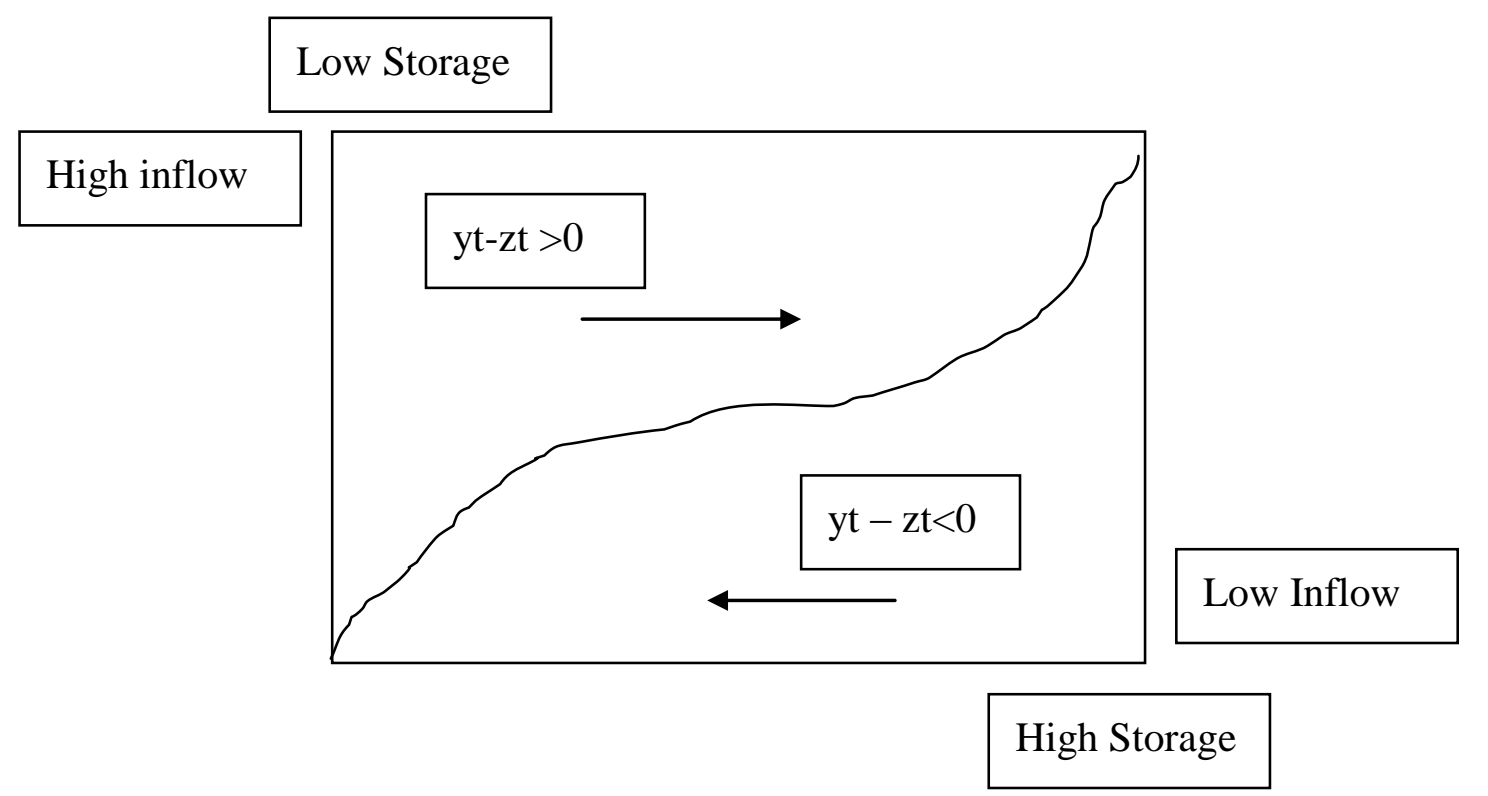

A simple policy that embodies the property we need is described as using our defined orderings of variables.

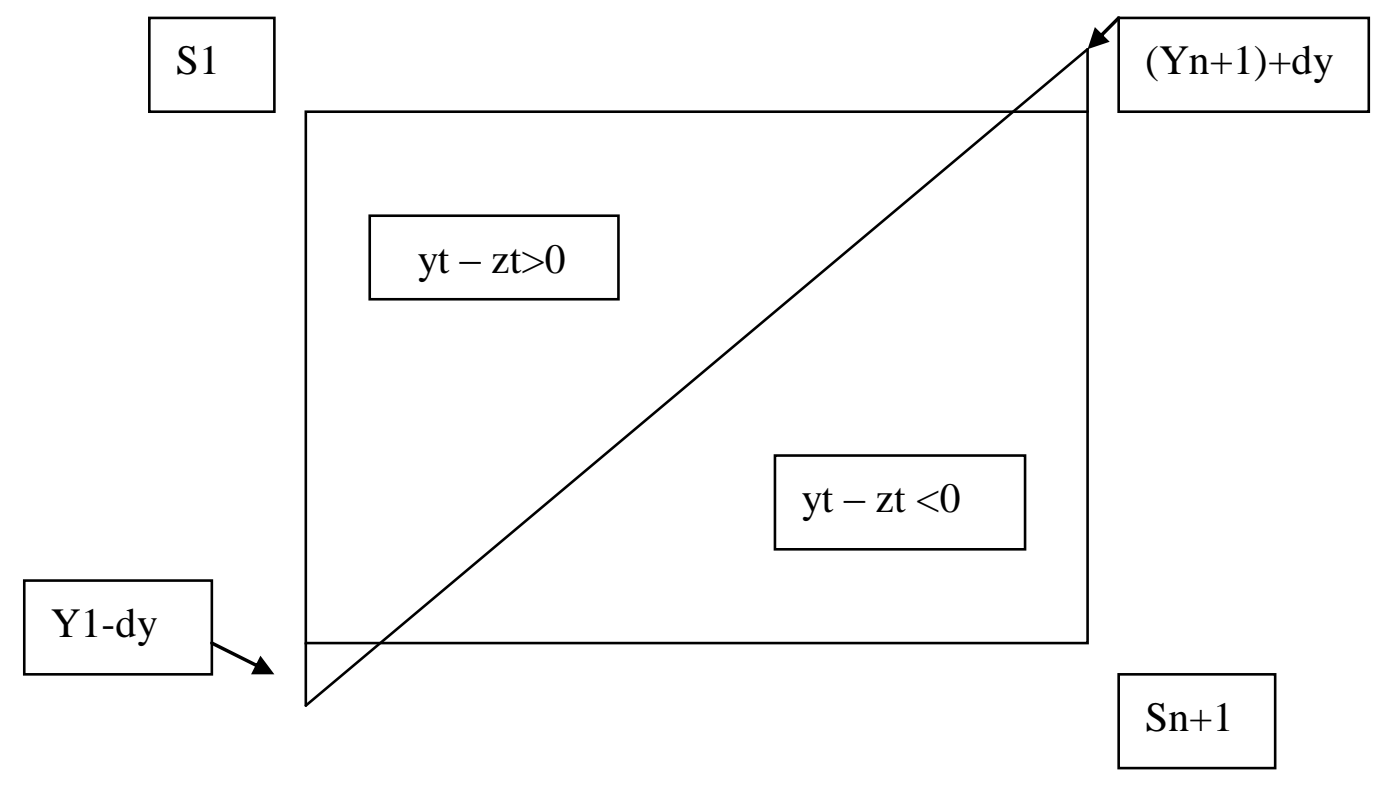


Along the straight-line $\left[\left(s_{1}, y_{1}-d y\right),\left(s_{n+1}, y_{n+1}+d y\right)\right]$ water coming from the inflow will be stored. For the combination of $S$ and $y$ in the grid that's on the left hand of the line, less water than the inflow will be used and some will be stored. However, for the combination of $s$ and $y$ in the grid that's on the right hand side of the line, the social planner will choose to use more hydro than the inflow which involves lowering the lake.

Presume that the function that represents the initial hydro generation policy is in the form of $z(s, y)=a+b s+c y$, we need to find out the expression for $a, b$ and $c$ that gives the properties stated above.

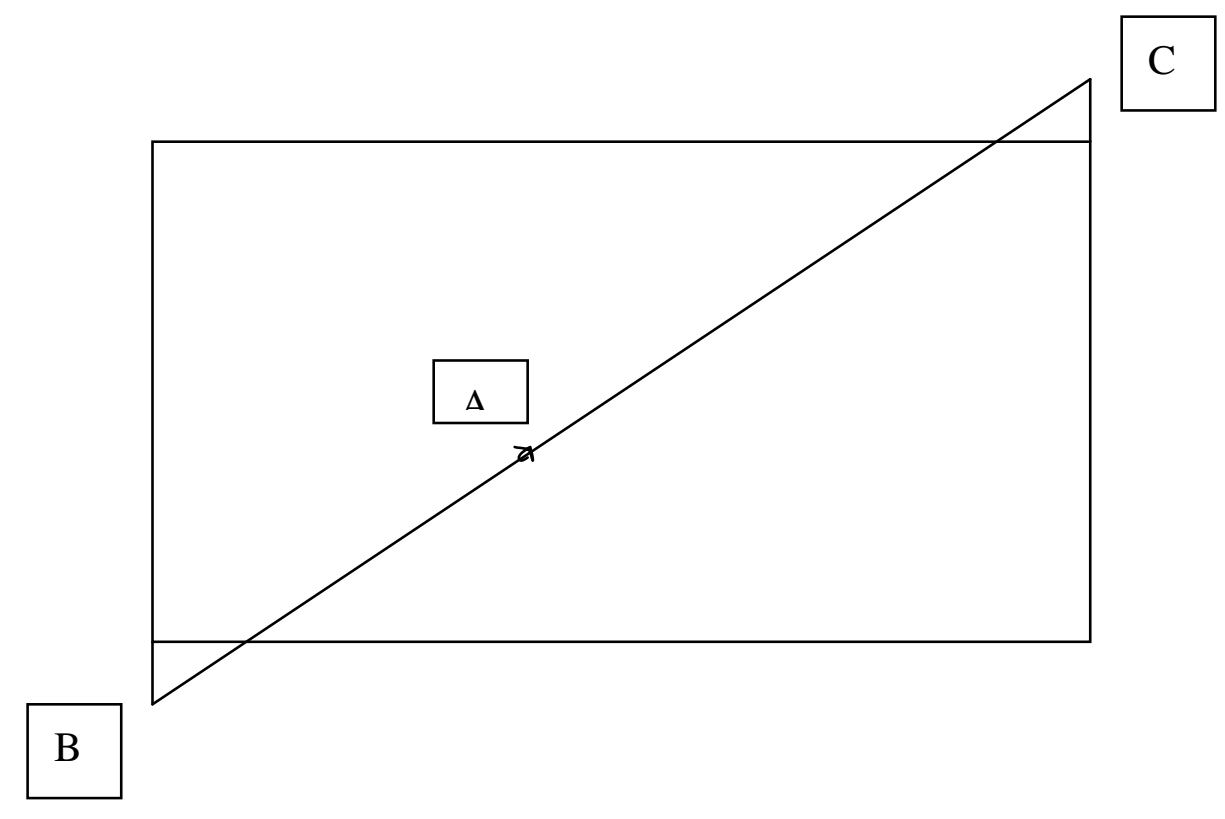

Since line from $\mathrm{B}$ to $\mathrm{C}$ that represents $y_{t}=z_{t}$ is a straight line, taking an arbitrary point A on this line, the slope of the line BA should be the same as the slope of line BC. Given this, the following equation has to hold:

$$
\frac{y-\left(y_{\min }-d y\right)}{s-s_{\min }}=\frac{y_{\max }+d y-\left(y_{\min }-d y\right)}{s_{\max }-s_{\min }}
$$

The expression for $\mathrm{s}$ that satisfies the above condition:

$$
s=\frac{d y \cdot s_{\max }+d y \cdot s_{\min }+s_{\max } \cdot y-s_{\min } \cdot y+s_{\min } \cdot y_{\max }-s_{\max } \cdot y_{\min }}{2 d y+y_{\max }-y_{\min }}
$$


Knowing $z(s, y)=a+b s+c y$ and when $z=y$ the level of storage $\mathrm{s}$ has to equal to the function above, the following equation has to hold:

$$
\begin{aligned}
z=a+y(c+ & \left.\frac{b \cdot s_{\max }}{2 d y+y_{\max }-y_{\min }}-\frac{b \cdot s_{\min }}{2 d y+y_{\max }-y_{\min }}\right)+\frac{b \cdot d y \cdot s_{\max }}{2 d y+y_{\max }-y_{\min }} \\
& +\frac{b \cdot d y \cdot s_{\min }}{2 d y+y_{\max }-y_{\min }}+\frac{b \cdot s_{\min } \cdot y_{\max }}{2 d y+y_{\max }-y_{\min }}-\frac{b \cdot s_{\max } \cdot y_{\min }}{2 d y+y_{\max }-y_{\min }}
\end{aligned}
$$

Given that along the line BC there's no change in the level of storage $(z=y)$, the coefficient of $y$ has to equal to 1 , and the intercept term has to equal to 0 .

$$
\begin{gathered}
c+\frac{b \cdot s_{\max }}{2 d y+y_{\max }-y_{\min }}-\frac{b \cdot s_{\min }}{2 d y+y_{\max }-y_{\min }}=1 \\
a+\frac{b \cdot d y \cdot s_{\max }}{2 d y+y_{\max }-y_{\min }}+\frac{b \cdot d y \cdot s_{\min }}{2 d y+y_{\max }-y_{\min }}+\frac{b \cdot s_{\min } \cdot y_{\max }}{2 d y+y_{\max }-y_{\min }} \\
-\frac{b \cdot s_{\max } \cdot y_{\min }}{2 d y+y_{\max }-y_{\min }}=0
\end{gathered}
$$

Solving the two equations above simultaneously, we obtain expressions for a and $c$ as a function of $b$ :

$$
\begin{aligned}
& c=\frac{2 d y-b \cdot s_{\max }+b \cdot s_{\min }+y_{\max }-y_{\min }}{2 d y+y_{\max }-y_{\min }} \\
& a=\frac{-b \cdot d y \cdot s_{\max }-b \cdot d y \cdot s_{\min }-b \cdot s_{\min } \cdot y_{\max }+b \cdot s_{\max } \cdot y_{\min }}{2 d y+y_{\max }-y_{\min }}
\end{aligned}
$$

Given a particular value of $b$, as long as a and $c$ follow the expression described above, this will make sure the plane that represents the initial hydro generation policy in 3D space of $(s, y, z)$ will go though the $z=y$ line. 
However, we still have another constraint on the choice of hydro generation, that is the hydro generation has to be non-negative $(z \geq 0)$. We need to choose a particular level of $b$ that satisfies this condition. One way of doing this is to make an arbitrary assumption that on the left hand boundary of the grid (that is when the storage is at its lowest level) the amount of hydro generation equals to half of the water inflow. In other words, we assume that when the storage is empty, we use only half of the water inflow and store the other half. Because the inflow is always non-negative, this guarantees that no matter how high the shadow price of water is hydro generation will never fall below zero.

Given the imposed condition that when $s=s_{\min }, z=\frac{1}{2} y$, the following equation has to hold:

$$
\begin{gathered}
z=\frac{y}{2}=\frac{-b \cdot d y \cdot s_{\max }-b \cdot d y \cdot s_{\min }-b \cdot s_{\min } \cdot y_{\max }+b \cdot s_{\max } \cdot y_{\min }}{a d y+y_{\max }-y_{\min }}+b \cdot s_{\min } \\
+\frac{2 d y-b \cdot s_{\max }+b \cdot s_{\min }+y_{\max }-y_{\min }}{a d y+y_{\max }-y_{\min }} \cdot y
\end{gathered}
$$

To simplify our calculation, if we set $d y=y_{\min }$, the equation above can be simplified into:

$$
\frac{y}{2}=\frac{y\left(d y+b\left(-s_{\max }+s_{\min }\right)+y_{\max }\right.}{d y+y_{\max }}
$$

We can solve for the expression:

$$
b=\frac{d y+y_{\max }}{2\left(s_{\max }-s_{\min }\right)}
$$

Therefore our initial guess for the hydro generation policy takes the following form:

$$
z=a+b s+c y
$$


Where

$a$

$=\frac{-\frac{d y\left(d y+y_{\max }\right) s_{\max }}{2\left(s_{\max }-s_{\min }\right)}-\frac{d y\left(d y+y_{\max }\right) s_{\min }}{2\left(s_{\max }-s_{\min }\right)}-\frac{s_{\min }\left(d y+y_{\max }\right) y_{\max }}{2\left(s_{\max }-s_{\min }\right)}+\frac{s_{\max }\left(d y+y_{\max }\right) y_{\operatorname{mi}}}{2\left(s_{\max }-s_{\min }\right)}}{2 d y+y_{\max }-y_{\min }}$

$b=\frac{d y+y_{\max }}{2\left(s_{\max }-s_{\min }\right)}$

$c=\frac{2 d y+y_{\max }-\frac{s_{\max }\left(d y+y_{\max }\right)}{2\left(s_{\max }-s_{\min }\right)}-\frac{s_{\min }\left(d y+y_{\max }\right)}{2\left(s_{\max }-s_{\min }\right)}-y_{\min }}{2 d y+y_{\max }-y_{\min }}$

For example, when $d y=0.05, y_{\min }=0.05, y_{\max }=2, d s=0.1, s_{\min }=0$ and $s_{\max }=5$, using the formulas stated above we get $\mathrm{a}=0, \mathrm{~b}=1.025$ and $\mathrm{c}=0.5$. Consequently, the initial guess for the hydro generation policy is:

$$
z[s, y]=1.025 s+0.5 y
$$




\section{Appendix 3: Evaluating Shadow prices of water Ws for points on the Right and Left boundaries}

In this section we show the derivation of the formula we use to evaluate the shadow price Ws for the case when $s$ is at its lowest level (left-hand-side boundary points) and when $s$ is at its highest level (right-hand-side boundary points).

\section{Left Boundary}

For the points on the left-hand-side boundary $\left(s=s_{\min }\right)$, since no smaller values of $\mathrm{s}$ is available, we need to derive a formula for Ws that only involves using values of the function at those points that's greater or equal to $s_{\min }$.

We can approximate a function near a certain point if we know its value at that point and the values of its derivatives there. The expansion is called a Taylor Series Expansion of the function about that point $x$, which gives

$$
f(x+d x)=f(x)+f^{\prime}(x) d x+\frac{1}{2} f^{\prime \prime}(x) d x^{2}+\cdots \approx f(x)+f^{\prime}(x) d x+\frac{1}{2} f^{\prime \prime}(x) d x^{2},
$$

and equivalently,

$$
4 f(x+d x) \approx 4 f(x)+4 f^{\prime}(x) d x+2 f^{\prime \prime}(x) d x^{2} .
$$

Using the same method we can get an approximation for $f(x+2 d x)$ :

$$
f(x+2 d x) \approx f(x)+2 f^{\prime}(x) d x+2 f^{\prime \prime}(x) d x^{2}
$$

Eliminating the second order differential term $f^{\prime \prime}(x) d x^{2}$, we get:

$$
4 f(x+d x)-f(x+2 d x)=3 f(x)+2 f^{\prime}(x) d x
$$


Rearrange to solve for $f^{\prime}(x)$ :

$$
f^{\prime}(x)=\frac{4 f(x+d x)-f(x+2 d x)-3 f(x)}{2 d x}
$$

Therefore when $s_{i}=s_{\min }$

$$
W_{s}\left[s_{i}, y_{j}\right]=\frac{4 W\left[s_{i+1}, y_{j}\right]-W\left[s_{i+2}, y_{j}\right]-3 W\left[s_{i}, y_{j}\right]}{2 d s}
$$

\section{Right Boundary}

For the points on the right side boundary ( $s=s \max$ ), since there are no higher values of $s$ available we need to derive a formula for Ws that only involves using values of function at points less or equal to $s_{\max }$.

Using a Taylor Series Expansion we get

$$
\begin{aligned}
& f(x-d x) \approx f(x)-f^{\prime}(x) d x+\frac{1}{2} f^{\prime \prime}(x) d x^{2} \\
& 4 f(x-d x) \approx 4 f(x)-4 f^{\prime}(x) d x+2 f^{\prime \prime}(x) d x^{2}
\end{aligned}
$$

And

$$
f(x-2 d x) \approx f(x)-2 f^{\prime}(x) d x+2 f^{\prime \prime}(x) d x^{2}
$$

To eliminate the second order differential term $f^{\prime \prime}(x) d x^{2}$, we have 
$4 f(x-d x)-f(x-2 d x)=3 f(x)-2 f^{\prime}(x) d x$

Rearrange to solve for $f^{\prime}(x)$ as a function of $f(x), f(x-d x)$ and $f(x-2 d x)$

$$
f^{\prime}(x)=\frac{3 f(x)-4 f(x-d x)+f(x-2 d x)}{2 d x}
$$

Therefore when $s_{i}=s_{\max }$,

$$
W_{s}\left[s_{i}, y_{j}\right]=\frac{4 W\left[s_{i}, y_{j}\right]-W\left[s_{i-1}, y_{j}\right]-3 W\left[s_{i-2}, y_{j}\right]}{2 d s}
$$


Appendix 4: Estimating the parameters for water inflow that follows a squareroot process $^{30}$

It is assumed that the water inflow $y_{t}$ evolves according to the square-root process, which is presented in the following form:

$$
d y_{t}=\eta\left(\mu-y_{t}\right) d t+\sigma \sqrt{y_{t}} d \zeta_{t}
$$

where $\eta, \mu$ and $\sigma$ are constant, and $d \zeta_{t}$ is independently and normally distributed with mean of zero and variance of one.

This square-root process is one type of mean-reverting diffusion process that yields only nonnegative values. According to this process, water inflow $y_{t}$ has an unconditional mean of $\mu$ and variance of $\frac{\mu}{2 \eta} \sigma^{2}$. The term $\eta$ measures the rate of mean-reversion which is the rate at which this process is pulled back towards the mean parameter $\mu$.

Suppose that $f\left(y_{t}\right)$ is a smooth function of $y_{t}$, using Taylor Expansion we can write the process for $f\left(y_{t}\right)$ as

$$
d f=\frac{d f}{d y_{t}} d y_{t}+\frac{1}{2} \frac{d^{2} f}{d y_{t}{ }^{2}} d y_{t}{ }^{2}+\cdots,
$$

where the dots denote a remainder which is small enough to be ignored.

If $f\left(y_{t}\right)=\sqrt{y_{t}}$, the terms in equation [4.1] can be computed as follows:

\footnotetext{
${ }^{30}$ The approach described here is used in Guthrie and Evans 2007 (electricity)
} 


$$
\begin{aligned}
& \frac{d f}{d y_{t}}=\frac{1}{2 \sqrt{y_{t}}} \\
& d y_{t}=\eta\left(\mu-y_{t}\right) d t+\sigma \sqrt{y_{t}} d \zeta_{t} \\
& \begin{aligned}
\frac{d^{2} f}{d y_{t}^{2}}= & \frac{1}{4 y_{t} \sqrt{y_{t}}} \\
& d y_{t}^{2}=\left(\eta\left(\mu-y_{t}\right) d t+\sigma \sqrt{y_{t}} d \zeta_{t}\right)^{2} \\
& =\eta^{2}\left(\mu-y_{t}\right)^{2} d t^{2}+\sigma^{2} y_{t} d \zeta_{t}^{2}+2 \eta\left(\mu-y_{t}\right) \sigma \sqrt{y_{t}} d t d \zeta_{t}
\end{aligned}
\end{aligned}
$$

Given that $d t^{2}=0, d t d \zeta_{t}=0$ and $d \zeta_{t}^{2}=d t$,

$$
d y_{t}^{2}=\sigma^{2} y_{t} d t
$$

Substitute these terms into equation [4.1],

$$
\begin{gathered}
d \sqrt{y_{t}}=\left(\left(\frac{\eta \mu}{2}-\frac{\sigma^{2}}{8}\right) \frac{1}{\sqrt{y_{t}}}-\frac{\eta}{2} \sqrt{y_{t}}\right) d t+\frac{\sigma}{2} d \zeta_{t} \\
=\left(\frac{\eta \mu}{2}-\frac{\sigma^{2}}{8}\right) d t \frac{1}{\sqrt{y_{t}}}-\frac{\eta}{2} d_{t} \sqrt{y_{t}}+\frac{\sigma}{2} d \zeta_{t}
\end{gathered}
$$

We re-write this continuous process with discrete time step length of $\Delta t$,

$$
\sqrt{y_{t+1}}-\sqrt{y_{t}}=\left(\left(\frac{\eta \mu}{2}-\frac{\sigma^{2}}{8}\right) \Delta_{t}\right){\sqrt{y_{t}}}^{-1}-\frac{\eta}{2} \Delta_{t} \sqrt{y_{t}}+\varepsilon_{t+1},
$$

Where $\varepsilon_{t+1} \sim \mathrm{N}\left(0, \phi^{2}\right)$ and $\phi^{2}=\frac{\sigma^{2}}{4} \Delta t$.

Equation [2] can be re-arranged into,

$$
\sqrt{y_{t+1}}=\left(\left(\frac{\eta \mu}{2}-\frac{\sigma^{2}}{8}\right) \Delta_{t}\right){\sqrt{y_{t}}}^{-1}+\left(1-\frac{\eta}{2} \Delta_{t}\right) \sqrt{y_{t}}+\varepsilon_{t+1}
$$




$$
=\alpha_{1}{\sqrt{y_{t}}}^{-1}+\alpha_{2} \sqrt{y_{t}}+\varepsilon_{t+1}
$$

$$
\begin{gathered}
\text { Where } \quad \alpha_{1}=\left(\frac{\eta \mu}{2}-\frac{\sigma^{2}}{8}\right) \Delta_{t} \\
\alpha_{2}=1-\frac{\eta}{2} \Delta_{t} .
\end{gathered}
$$

We regress y mean of ordinary least square (OLS) $\sqrt{y_{t+1}}$ on the variables of ${\sqrt{y_{t}}}^{-1}$ and $\sqrt{y_{t}}$ to obtain estimates for coefficients $\hat{\alpha}_{1}, \hat{\alpha}_{2}$ and the standard error of estimation $\hat{\phi}$. Therefore given that the OLS estimators are consistent, the parameter estimates for the square root process are as follows,

$\hat{\eta}=\frac{2\left(1-\hat{\alpha}_{2}\right)}{\Delta t}$

$\hat{\mu}=\frac{\hat{\phi}^{2}}{2\left(1-\hat{\alpha}_{2}\right)}+\frac{\hat{\alpha}_{1}}{1-\hat{\alpha}_{2}}$,

$\hat{\sigma}=\frac{2 \hat{\phi}}{\sqrt{\Delta t}}$

The data we use to estimate the parameters for the water inflow $\left(y_{t}\right)$ process is the monthly water inflow data available from the centralised dataset issued by the New Zealand Electricity Commission. These inflow data are measured in the unit of gigawatt hour (GWh), and they cover the period from July 1931 to June 2006. 
We first divide all the inflow data by 24727 to convert them into standard units ( 1 standard unit $=24727$ gigawatt hours ${ }^{31}$ ), then annualize these monthly inflow data by multiplying them by 12 . We take the square root value of these annualized data and regress them on their first lag and their first lag inverse to obtain estimated parameters of $\hat{\alpha}_{1}, \hat{\alpha}_{2}$ and $\hat{\phi}$. Using these estimates we can compute the parameters of the square root process for water inflow $y_{t}$,

$$
d y_{t}=6.9448\left(1-y_{t}\right) d t+0.9056 \sqrt{y_{t}} d \xi_{t}
$$

${ }^{31}$ Details see section : New Zealand Calibration. 


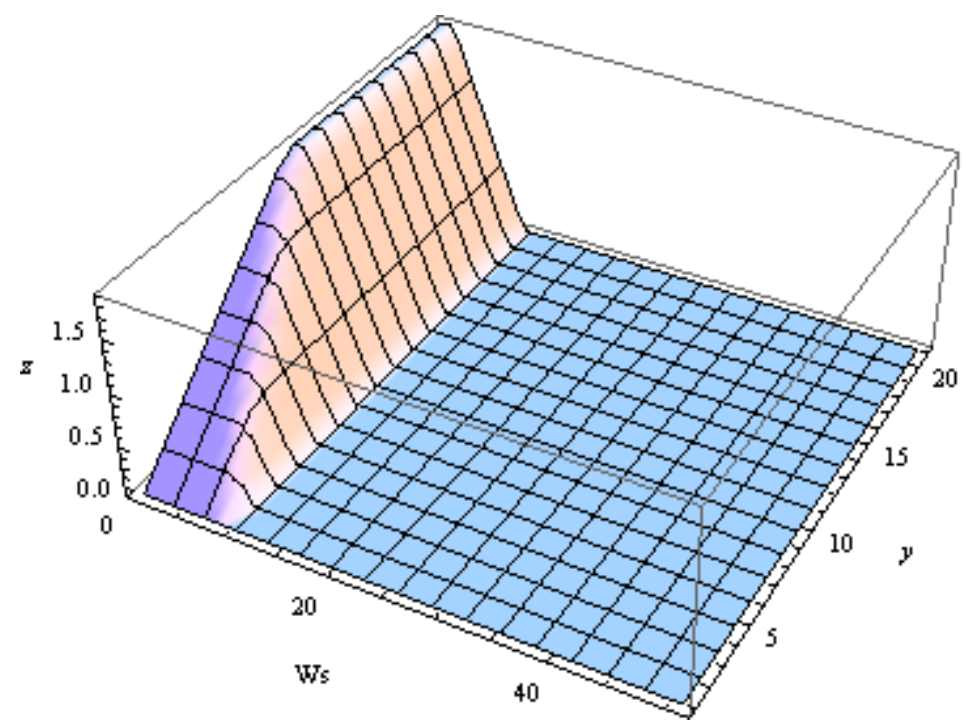

Diagram A5-1: Hydro generation $z^{*}$ for the social planner when the lake is empty

Diagram A5-1 shows the optimal hydro generation policy for a social planner when the lake is empty. Given inflow $y$, when the shadow price of water is zero, the social planner chooses to use as much of the inflow for electricity generation as possible, subject to the hydro electricity generation capacity constraint. Any excess water is stored. However, as the shadow price of water gets higher, the optimal level of hydro generation will gradually decrease until it reaches zero. This is because when the shadow price gets higher, the benefit of storing the water in the lake now for later use becomes greater than the benefit we get from using it to generate electricity at the present date. As a result when $W_{s}$ gets higher it is more likely that the social planner will store more water. When the shadow price is greater than 2 (the $10^{\text {th }}$ value for $W_{s}$ in our grid) ${ }^{32}$, the social planner will put all of the inflow into storage. The critical value of the shadow price $W_{s}$ above which the social planner starts to put water into storage depends on the amount of inflow from the river $y$. When the inflow is high, the

\footnotetext{
${ }^{32}$ About the set-up of the grid, see Appendix 2
} 
social planner will start to put water into the lake at a low level of $W_{s}$; whereas when the inflow is low, $W_{s}$ has to be sufficiently high for storage to occur. The peak electricity price is more likely to occur in this scenario because the scarcity of water (no water in storage) will be associated with a high shadow price of water.

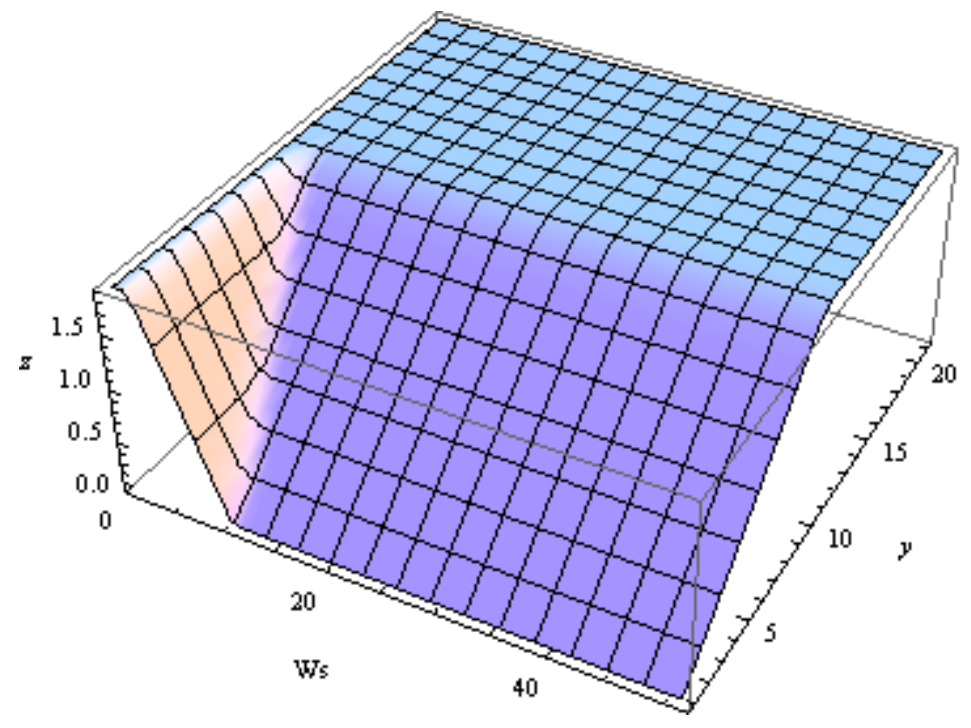

Diagram A5-2: Hydro generation $z^{*}$ for the social planner when the Lake is full

Diagram A5-2 shows the optimal hydro generation policy when the lake is full. Since the storage option is no longer available, any inflow from the river must either be used in generation or spilled. Stored water is costly only when hydro generation exceeds the amount of inflow. When the inflow is high, it is optimal for the social planner to run hydro electricity generation at maximum generation capacity and spill the remaining inflow. This is because in such cases the water resource is so abundant that the unconstrained optimal hydro generation exceeds the capacity constraint. On the other hand when the water inflow is low, the optimal amount of hydro generation depends on the shadow price of water. When the shadow price of water is high, it is too costly to use water in the storage lake to generate; therefore it is optimal for the social planner to set the 
amount of hydro generation equal to the water inflow in that period. However, when the shadow price is low, it becomes optimal for the social planner to lower the storage lake by allocating stored water to hydro generation.

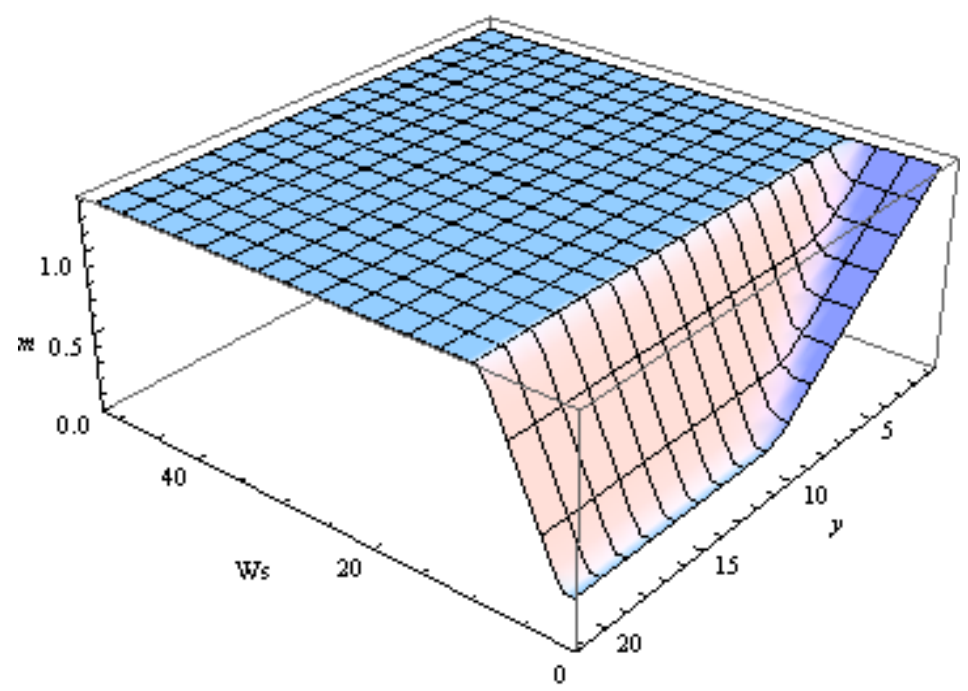

Diagram A4-3: Gas generation $\mathrm{m}^{*}$ for the social planner given that the lake is empty

Diagram A4-3 shows the optimal gas generation policy of the social planner when the lake is empty. When the shadow price of water equals zero, at inflow levels that are above the amount of water needed for reaching the maximum generation capacity of the hydro plant the social planner chooses to run hydro generation at its full generation capacity which equals 1.901 units and sets the gas generation at its corresponding optimal level which equals 0.242896 units. This positive quantity of gas generation at zero shadow price of water comes from the assumption that gas-generated electricity has a lower transmission cost relative to hydro-generated electricity ${ }^{33}$ and it has a linear marginal cost function starting from zero ${ }^{34}$. As a result no matter what value the shadow price of water is, the first 0.242896 units of electricity on the electricity supply curve will always be generated using gas plant. On the other hand, when the shadow price equals

\footnotetext{
${ }^{33}$ Transmission cost for hydro generated electricity is at 0.19363 per unit, whereas the transmission cost for gas generated electricity is at 0.10106 per unit. For details, see section xxx.

${ }^{34}$ The gas plant has a total cost function of $C(m)=0.7112 m^{2}$, and a marginal cost function of $M C(m)=$ $1.4224 \mathrm{~m}$.
} 
zero, for the inflow level that is below 1.901 the social planner uses all of the inflow in hydro generation. Being a substitute for hydro generation, gas generation will be at the level which maximizes the net social benefit given that all the inflow is used in hydro generation. This optimal gas generation quantity decreases as inflow increases. At a given level of inflow $y$, as the shadow price of water $W_{s}$ increases gas generation is increasingly preferred to hydro generation as in that case it is cheaper to use gas than water and gas generation is substituted for hydro. That is why Diagram 5-4 shows that as $W_{s}$ increases the amount of gas generation increases until it reaches its interior optimal generation level given that gas is the sole source of electricity generation $(m=1.29143)$. These observations show that if the shadow price of water is high enough, gas is capable of being the sole generation on the market; whereas hydro will never be the sole generation as at least 0.242896 units of gas generation will always take place due to its low transmission cost.

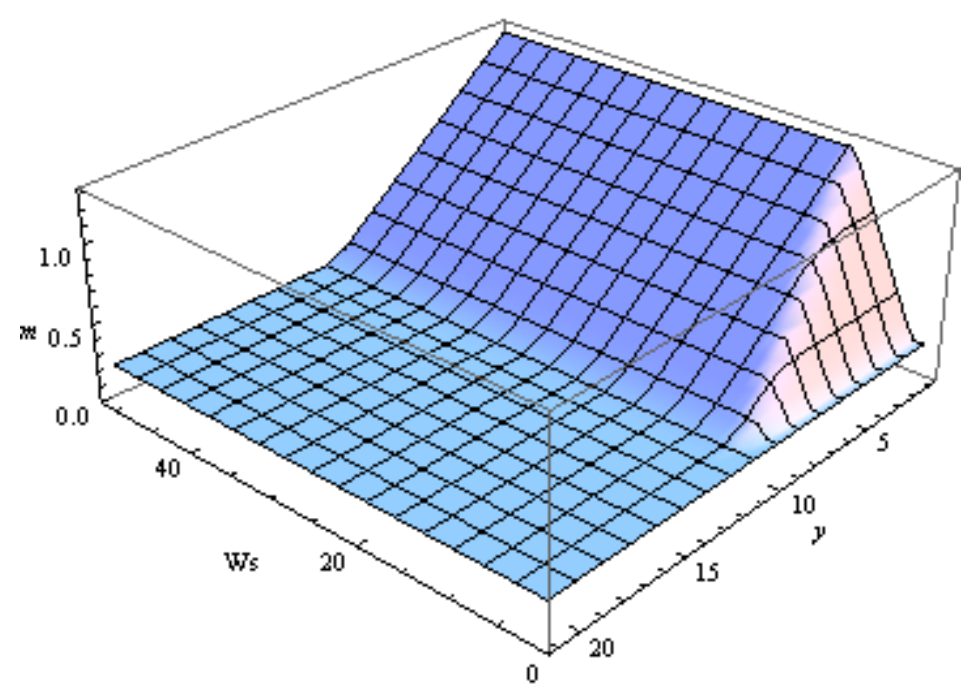

Diagram A5-4: Gas generation $\mathrm{m}^{*}$ for the social planner when the Lake is full

Diagram A5-4 shows the optimal gas generation policy of a social planner when the lake is full. When inflow is high (inflow is higher than 2 , or the $10^{\text {th }}$ value for 
y), the water resource is so abundant that the social planner will meet the demand by running hydro plant at its full capacity and use gas plant to generate 0.242896 units of electricity. The same happens when the shadow price of water equals zero while the water inflow is low, as in such case the water is very cheap so that it is optimal for the social planner to substitute as much hydro generation as possible for gas. However, given that the inflow is low, when the opportunity cost of water $W_{s}$ is higher, the producer will start to use gas generation. At a given level of $W_{s}$, generally a decrease in inflow $y$ will lead to an increase in the level of gas generation until it reaches its unconstrained optimal generation level (which is lower than the maximum generation capacity of the gas plant, $\left(m^{*}=1.29143<1.33454=\bar{m}\right)$. 


\section{Appendix 6: Static optimization result for the monopoly model}

In a static setting, a monopolist electricity generator chooses the amount of hydro and gas generation to maximize its profit while the water inflow and the shadow price of water are taken as given. The objective function for the electricity generator in a monopoly market is given below,

$$
\max _{m_{t}, z_{t}} f=\varphi\left(m_{t}+z_{t}\right) \cdot\left(m_{t}+z_{t}\right)-z_{t} \frac{\partial W}{\partial s}-C\left(m_{t}\right)-k_{1} z_{t}-k_{2} m_{t}
$$

The monopoly generator's optimal generation policies in this static setting will be summarized by six diagrams, which cover the optimal generation policy for both hydro and gas generation while the lake is empty, full or somewhere in between.

(1) Optimal hydro generation when the lake is empty $\left(z^{*} \mid S_{t}=0\right)$;

(2) Optimal hydro generation when the lake has spare capacity $\left(z^{*} \mid 0<S_{t}<\bar{S}\right)$

(3) Optimal hydro generation when the lake is full $\left(z^{*} \mid S_{t}=\bar{S}\right)$;

(4) Optimal gas generation when the lake is empty $\left(m^{*} \mid S_{t}=0\right)$;

(5) Optimal gas generation when the lake has spare capacity $\left(m^{*} \mid 0<S_{t}<\bar{S}\right)$; and

(6) Optimal gas generation when the lake is full $\left(m^{*} \mid S_{t}=\bar{S}\right)$. 


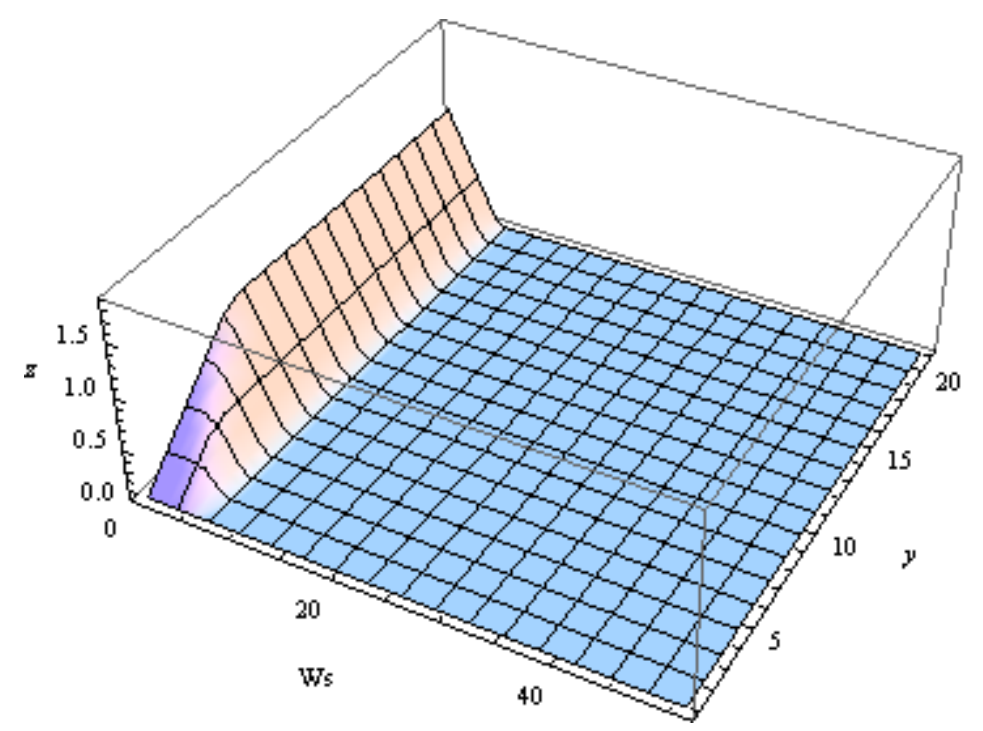

Diagram A6-1: Hydro generation $z$ * for a monopoly generator when the lake is empty

Diagram A6-1 shows the optimal hydro generation policy for a monopolist generator when the lake is empty. The hydro generation capacity constraint is never binding here, because the generator's optimal level hydro generation at any given combination of shadow price of water and inflow is always less than the maximum generation capacity. For a monopolist generator, if there is no constraint on the availability of water resources, his optimal level of hydro generation is 1.07645. Given inflow $y$, when the shadow price of water is zero, the monopolist generator chooses to generate the optimal level of hydro generation of 1.07645 as long as there is enough inflow from the river and store the excess water. If the inflow in that period is less than 1.07645, the generator uses all inflow in hydro generation without storing any. However, as the shadow price of water gets higher, the optimal level of hydro generation will gradually decrease until it reaches zero. As shadow price gets higher, the benefit of storing the water in the lake for later use becomes greater than the benefit we get from using it to generate electricity at the present date. As a result when Ws is higher it is more likely that the monopolist generator will store more water. When the shadow price is greater than 1.2 (the $7^{\text {th }}$ value for $\left.W s\right)^{35}$, the

\footnotetext{
${ }^{35}$ This is equivalent to $\mathrm{xxx}$ per MWh.
} 
monopolist generator will put all of the inflow into storage. The critical value of the shadow price Ws above which the monopolist generator starts to put water into storage depends on the amount of inflow from the river $y$. When the inflow is greater than the un-constrained optimal level of hydro generation, the monopoly generator will put water into the lake at any given value of $W s$ (including when $W s=0$ ); whereas when the inflow is less than 1.07645, Ws has to be sufficiently high for storage to occur and the smaller the inflow the larger the critical $W s$ required for adding water into storage to take place. The peak electricity price is more likely to occur in this scenario because of the scarcity of water (no water in storage).

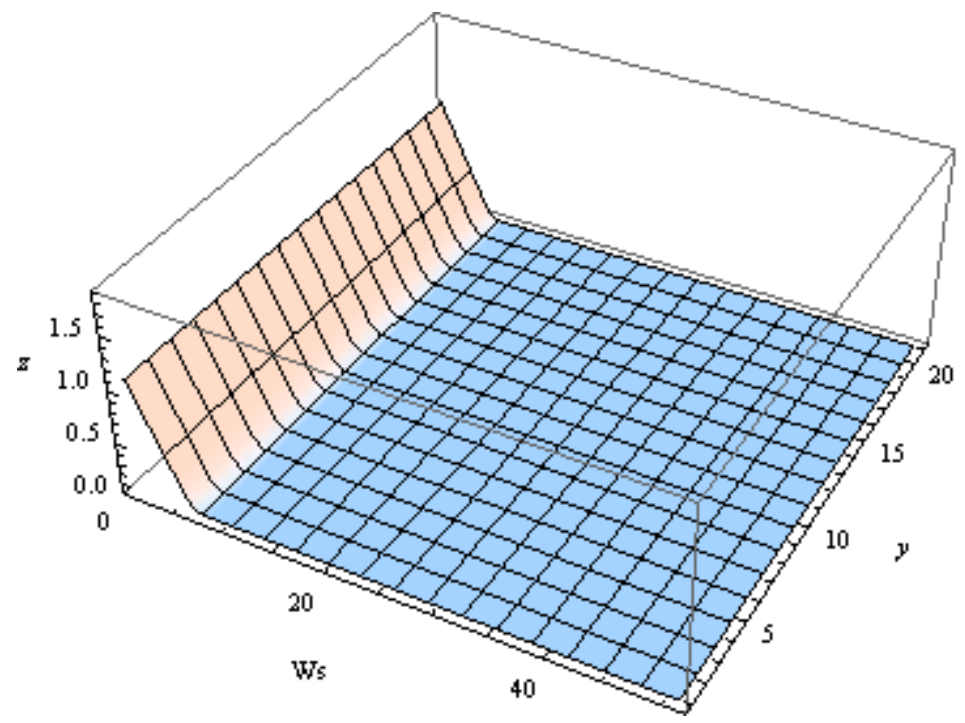

Diagram A6-2: Hydro generation $z$ for a monopoly generator when the lake is partially full

Diagram A6-2 shows the optimal hydro generation policy for a monopolist generator when there is spare storage capacity in the lake, that is when the lake is neither empty nor full. At a point in time, there is water inflow from the river and stored water in the lake, thus no upper bound is imposed on hydro generation apart from the capacity of the plant. As a result, the optimal hydro generation policy only depends on the shadow price of water $W_{s}$ : it is not a function of the inflow level. When the shadow price of water $W s$ is very low at a 
value that is close to zero, we observe that the monopoly generator chooses the amount of hydro generation equal to its un-constrained optimal level which is 1.07645. As Ws moves away from zero to higher values, the opportunity cost of using hydro resource in electricity generation increases, and it is optimal for the generator to decrease the level of hydro generation $\left(z^{*}\right)$. This decline in $z^{*}$ will continue as $W s$ increases until hydro generation reaches zero (when $W s$ takes value beyond 1.2 , the $7^{\text {th }}$ value of $W s$ in our grid).

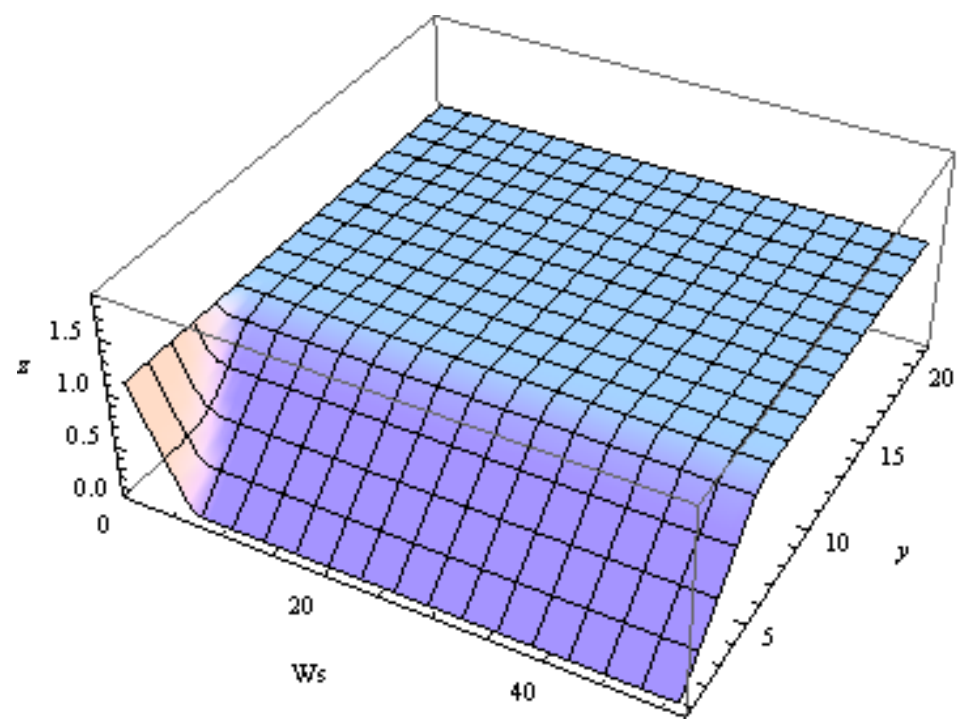

Diagram A6-3: Hydro generation $z$ * for a monopoly generator when the lake is full

Diagram A6-3 shows the optimal hydro-generation policy for a monopolist generator when the lake is full. Since the storage option is no longer available, any inflow from the river must either be used in generation or spilled. Using water is costly only when hydro generation exceeds the amount of inflow. When the inflow is higher than 1.07645 , it is optimal for the generators to run hydro electricity generation at the un-constrained optimal generation level $\left(z^{*}=1.07645\right.$, which is lower than its maximum generation capacity) and spill the remaining inflow. On the other hand when the water inflow is less than 1.07645, the optimal amount of hydro generation depends on the shadow price of water. When the shadow price of water is high, it is too costly to use water in 
the lake to generate; therefore it is optimal for the generator to set the amount of hydro generation equal to the water inflow in that period. However, when the shadow price is low, it becomes optimal for the generators to lower the lake by allocating stored water to hydro generation.

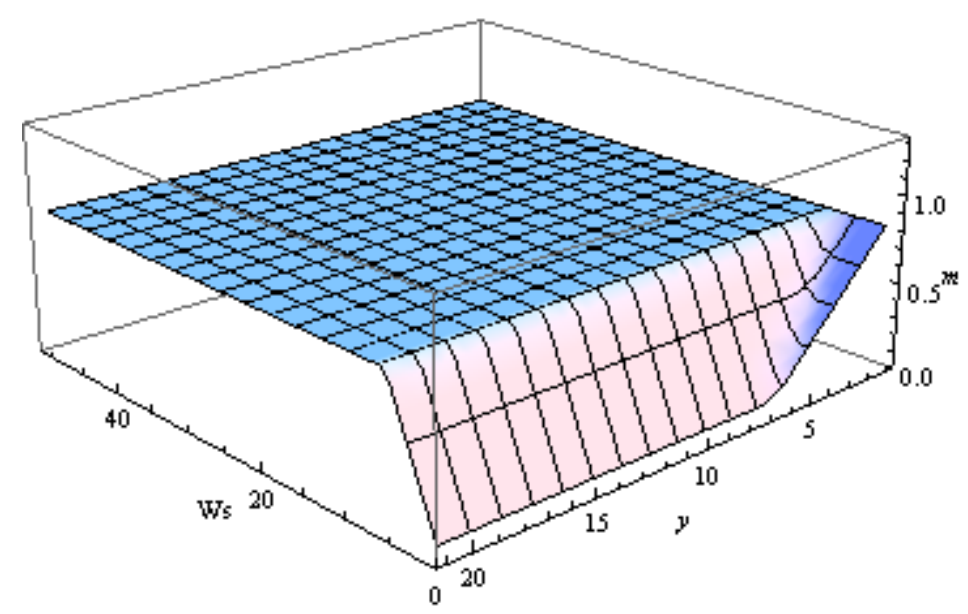

Diagram A6-4: Gas generation $m *$ for a monopoly generator when the lake is empty

Diagram A6-4 shows the optimal gas generation policy for a monopoly generator when the lake is empty. When the shadow price of water is equal to zero, at inflow levels that are above 1.07645 the generator chooses to generate hydro generation at its unconstrained optimal level and sets the gas generation equal to 0.0669994 units. This positive quantity comes from the assumption that gas generated electricity has a lower transmission cost relative to hydro generated electricity and a linear marginal cost function that starts from zero. As a result no matter what value the shadow price of water is, the first 0.0669994 units of electricity in this market will always be generated using gas plant. Whereas for the inflow levels that are below 1.0821 the generator uses all of the inflow in hydro generation. Being a substitute for hydro generation, gas generation will be at the level which maximizes the profit for the generator given that all the inflow is used in hydro generation. This optimal generation quantity decreases as inflow (which equals to the amount of hydro generation) 
increases. At a given level of inflow $y$, as the shadow price of water $W s$ increases gas generation is increasingly preferred to hydro generation as in that case it is relatively cheaper to use gas than water and gas generation is substituted for hydro. That is why Diagram 5-24 has as Ws increasing the amount of gas generation increases until it reaches its unconstrained optimal generation level $\left(m^{*}=0.832338\right)$ which is lower than the maximum generation capacity of the gas plant $(\bar{m}=.133454)$.

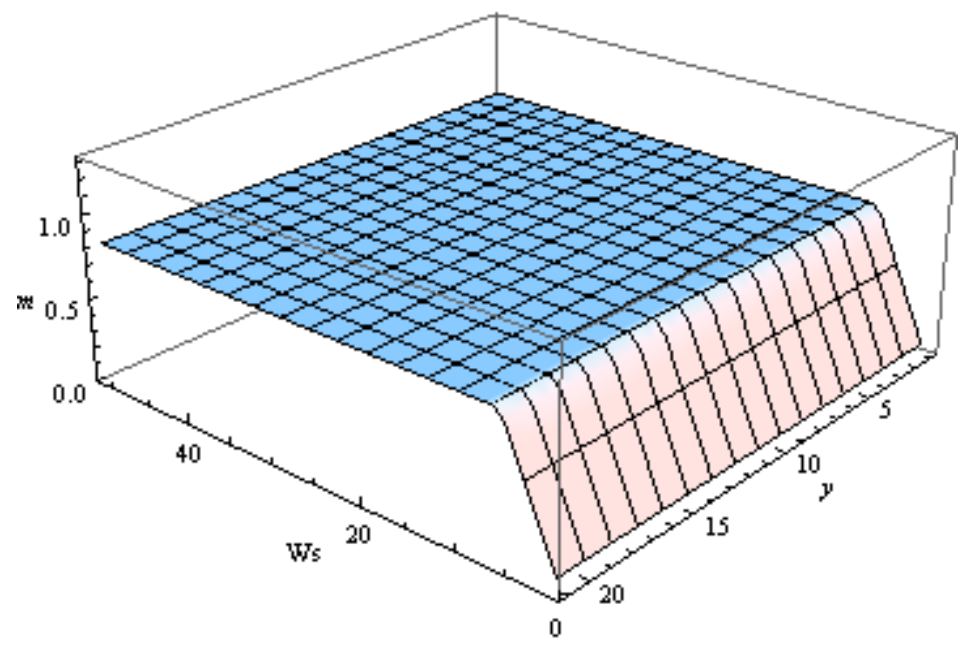

Diagram A6-5: Gas generation $m *$ for the monopoly generator when the lake is partially full

Diagram A6-5 shows the optimal gas generation policy for the monopolist generator when the lake is neither empty nor full. At a point in time, the optimal electricity generation policy only depends on the shadow price of water $W s$ : it is not a function of the inflow level. When the value of stored water $W s$ equals zero, the monopolist generator chooses to generate 0.0669994 units of electricity using the gas plant. That is the optimal level of gas generation given that the hydro plant is generating at its unconstrained optimal level (given that the shadow price of water equals zero). As the shadow price of water increases from zero the monopolist generator will choose to substitute more gas for hydro, until the unconstrained optimal level of gas generation, which is equal to 
0.832338 units, is reached. The minimum value of the shadow price $W s$ in which the gas becomes the sole source of electricity generation is 1.2 (which is the $7^{\text {th }}$ value of $W s$ in our grid).

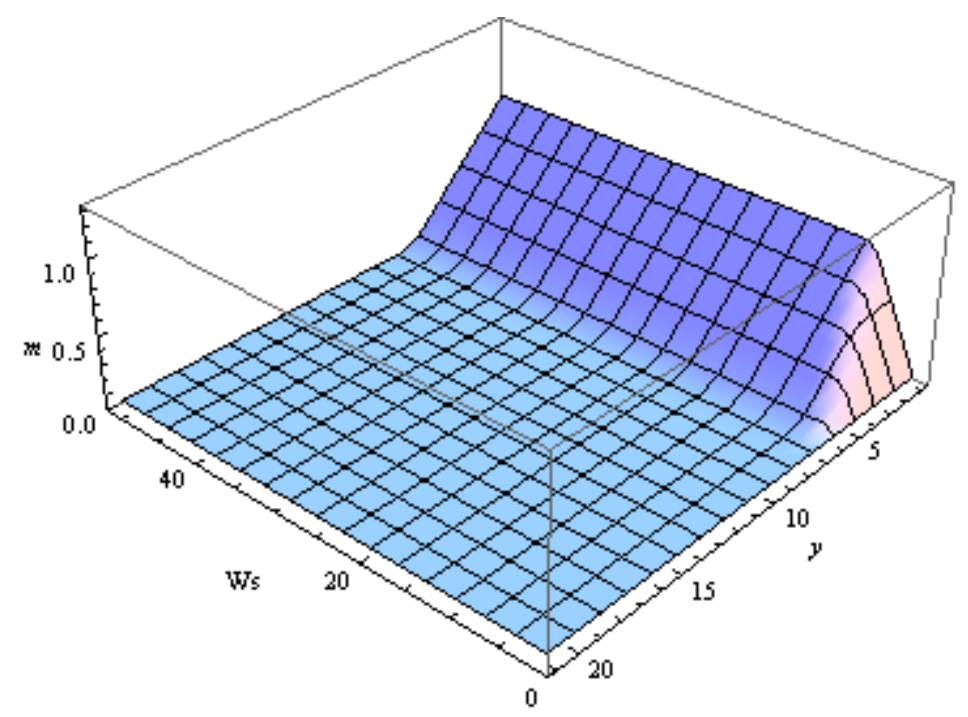

Diagram A6-6: Gas generation $m^{*}$ for a monopolist generator when the lake is full

Diagram A6-6 shows the optimal gas generation policy when the lake is full. When inflow is high (inflow is higher than 1.07645, which is slightly below the $7^{\text {th }}$ value for $y$ in Diagram 5-26), the water resource is so abundant that the generator will set hydro generation at its unconstrained optimal level $\left(z^{*}=1.07645\right)$ and therefore choosing the gas at its corresponding optimal level of 0.0669994 units. The same happens when the shadow price of water equals zero while the water inflow is less than 1.07645 , as in this case the stored water is worth nothing so that it is optimal for the generator to set the hydro generation at its unconstrained optimal level and generate only a small quantity of electricity using gas generation. However, given that the inflow is low and the opportunity cost of water $W_{s}$ is greater than zero, the producer will start to use more gas generation. At a given level of $W_{s}$, generally a decrease in inflow $y$ 
will lead to an increase in the level of gas generation until it reaches its unconstrained optimal generation level at 0.832338 units. 